CENTRO UNIVERSITÁRIO FEI

CESAR AUGUSTO BELCHIOR DE CARVALHO

\title{
AVALIAÇÃO DA DISTORÇÃO HARMÔNICA DE NANOFIOS TRANSISTORES EMPILHADOS
}

São Bernardo do Campo 
CESAR AUGUSTO BELCHIOR DE CARVALHO

\section{AVALIAÇÃO DA DISTORÇÃO HARMÔNICA DE NANOFIOS TRANSISTORES EMPILHADOS}

Dissertação de Mestrado apresentada ao Centro Universitário FEI, como parte dos requisitos necessários para obtenção do título de Mestre em Engenharia Elétrica. Orientado pelo Prof. Dr. Marcelo Antonio Pavanello.

São Bernardo do Campo 
Carvalho, Cesar Augusto Belchior de.

Avaliação da distorção harmônica de nanofios transistores empilhados / Cesar Augusto Belchior de Carvalho. São Bernardo do Campo, 2021. 102 f. : il.

Dissertação - Centro Universitário FEI.

Orientador: Prof. Dr. Marcelo Antonio Pavanello.

1. Nanofios empilhados. 2. Distorção harmônica. 3. Amplificadores operacionais. 4. Porta circundante. 5. Porta tripla. I. Pavanello, Marcelo Antonio, orient. II. Título.

Elaborada pelo sistema de geração automática de ficha catalográfica da FEI com os dados fornecidos pelo(a) autor(a). 
Aluno: Cesar Augusto Belchior de Carvalho

Matrícula: 119102-2

Título do Trabalho: Avaliação da Distorção Harmônica de Nanofios Transistores Empilhados..

Área de Concentração: Nanoeletrônica e Circuitos Integrados

Orientador: Prof. Dr. Marcelo Antonio Pavanello

Data da realização da defesa: 31/05/2021

\section{ORIGINAL ASSINADA}

\section{Avaliação da Banca Examinadora:}

A defesa pública de dissertação foi realizada virtualmente, com a presença de todos os membros da Banca Examinadora em toda a sua duração. A defesa foi iniciada com a apresentação da dissertação pelo candidato. Em seguida, ocorreu a arguição por

todos os membros da banca. O candidato respondeu as questões efetuadas pelos membros com sucesso. Os comentários recebidos dos membros durante a avaliação serão incorporados à dissertação, gerando um novo volume. Em sessão fechada,

a Banca Examinadora deliberou pela aprovação unânime da dissertação e outorga do título de Mestre.

São Bernardo do Campo, 31 / 05 / 2021.

\section{MEMBROS DA BANCA EXAMINADORA}

Prof. Dr. Marcelo Antonio Pavanello

Prof.a Dr.a Lígia Martins D'Oliveira

Prof. Dr. Alberto Vinicius de Oliveira
Ass. :

Ass.

Ass. :

A Banca Julgadora acima-assinada atribuiu ao aluno o seguinte resultado:
APROVADO $\bigotimes$
REPROVADO

\section{VERSÃO FINAL DA DISSERTAČ̃̃O} APROVO A VERSÃO FINAL DA DISSERTAÇÃO EM QUE FORAM INCLUÍDAS AS RECOMENDAÇÕES DA BANCA EXAMINADORA
Aprovação do Coordenador do Programa de Pós-graduação

Prof. Dr. Carlos Eduardo Thomaz 
Dedico este trabalho à minha família, que tanto me apoia em todos os meus projetos, trabalhos e sonhos. 


\section{AGRADECIMENTOS}

Agradeço a meus pais, colegas, professores, mestres, amigos e a todos os que sempre me apoiaram em minha busca pelo conhecimento e pela contribuição científica. Agradeço imensamente ao meu orientador, Marcelo Pavanello, que com muita atenção e paciência sempre teve êxito em coordenar com maestria minha jornada acadêmica.

Muito Obrigado ao Centro Universitário FEI, que desde a graduação valoriza meus esforços, acreditando e investindo em minha formação. 
Não podemos prever o futuro, mas podemos criá-lo. (Peter Drucker) 


\section{RESUMO}

Este trabalho estuda as características não lineares dos transistores nanofios empilhados, implementados em tecnologia SOI (silicon-on-insulator), operando como amplificadores operacionais de um único transistor. São estudadas as influências da largura do nanofio, do comprimento de canal e do nível de inversão em que os nanofios são polarizados. A não linearidade é especialmente relevante para as aplicações analógicas, cujos sinais de saída podem sofrer distorções em decorrência do desempenho do componente, comprometendo a transmissão e/ou amplificação dos sinais. Após realizar uma análise evolutiva das tecnologias, o trabalho demonstra que a arquitetura do dispositivo estudado está composta em dois níveis, sendo um transistor de porta tripla (trigate) e um transistor de porta circundante (gate-allaround). A revisão bibliográfica aborda alguns dos parâmetros elétricos de maior importância no estudo dos transistores, conceituando-os fisicamente e expondo suas equações características. A primeira etapa do trabalho ainda apresenta algumas das propriedades analógicas investigadas previamente, tais como a tensão de limiar, corrente de dreno, efeito de corpo, inclinação de sublimiar e a transcondutância, indicando a superioridade dos nanofios empilhados em relação ao SOI de porta única em alguns aspectos, sobretudo na capacidade de fornecimento de corrente de dreno, o que está alinhado com o aumento da possibilidade de integração da tecnologia tão almejada pelo setor mercadológico. $\mathrm{O}$ trabalho se dedica a apresentar a distorção harmônica e seus efeitos em circuitos e sistemas elétricos, indicando que o fenômeno possui grande importância em áreas diversas, sendo influente tanto em um único amplificador como em instalações elétricas e equipamentos indústriais mais complexos. Para obter este parâmetro no objeto de interesse, foi utilizado o método da função integral (IFM), que permite adquirir os dados apenas com a curva da corrente de dreno em função da tensão de porta ( $\mathrm{I}_{\mathrm{DS}} \mathrm{x} \mathrm{V}_{\mathrm{GS}}$ ) extraída experimentalmente, eliminando-se a necessidade de medições de corrente alternada (AC), que podem trazer ruídos mais difíceis de serem dissociados do sinal real dada a magnitude da corrente do dispositivo nanométrico. Os resultados demonstram que a distorção harmônica dos nanofios empilhados é majoritariamente relacionada ao harmônico de segunda ordem (HD2), cujo valor se distancia em 30 dB do terceiro harmônico. Os dados apresentados indicam que os nanofios com maiores $\mathrm{W}_{\mathrm{FIN}}$ possuem maior não linearidade e menor ganho de tensão em malha aberta: em relação ao conjunto de transistores com comprimento fixo $\mathrm{L}=100 \mathrm{~nm}$, a maior distorção harmônica se deu para o componente com largura $\mathrm{W}_{\mathrm{FIN}}=40 \mathrm{~nm}$, que apresentou distorção harmônica total THD $\approx-19 \mathrm{~dB}$ e ganho em malha aberta $\mathrm{A}_{\mathrm{v}} \approx 38 \mathrm{~dB}$ para maiores valores de $\mathrm{g}_{\mathrm{m}} / \mathrm{I}_{\mathrm{DS}}$, operando em inversão moderada. Já 
para os transistores nanofios com largura fixa de $W_{\text {FIN }}=10 \mathrm{~nm}$, foi possível observar que a maior não linearidade ocorre em transistores com maiores comprimentos: para os nanofios empilhados de $\mathrm{L}=400 \mathrm{~nm}$, obteve-se THD $\approx-19 \mathrm{~dB}$ em maiores valores de $\mathrm{g}_{\mathrm{m}} / \mathrm{I}_{\mathrm{DS}}$. A relação obtida entre as dimensões físicas dos transistores e a distorção harmônica são justificadas através das equações matemáticas apresentadas, sendo que a correlação entre os maiores resultados de THD para os nanofios com maiores $\mathrm{W}_{\text {FIN }}$ também é explicada a partir da maior variação de $\mathrm{dg}_{\mathrm{m}} / \mathrm{dV}_{\mathrm{GT}}$, em acordo com resultados da literatura observados para nanofios com um único nível. Ao fim do trabalho, novas análises são realizadas para um novo conjunto de dados, formados por nanofios com múltiplos fins. As maiores magnitudes de corrente de dreno permitiram evidenciar os resultados e confirmar, com maior grau de confiabilidade, a relação entre as não-linearidades e as características dimensionais dos nanofios, uma vez que os dados trazem uma média de resultados de diversos dispositivos operando em paralelo.

Palavras-chave: Nanofios empilhados. Distorção harmônica. Amplificadores operacionais. Porta circundante. Porta tripla. Tecnologia SOI. 


\begin{abstract}
This work studies the nonlinear characteristics of stacked nanowire transistors, implemented in SOI (silicon-on-insulator) technology, operating as operational amplifiers of a single transistor. The influence of the nanowire width, channel length and inversion level at which the nanowires are biased are studied. The effect is especially relevant for analog applications, whose output signals may suffer distortions due to the component's performance, compromising the transmission and/or amplification of the signals. After conducting an analysis of the technology's evolution, the research shows that the studied device's architecture is composed of two levels, being a trigate transistor and a GAA (gate-all-around). The literature review addresses some of the most important electrical parameters in the study of transistors, conceptualizing them physically and exposing their characteristic equations. The first stage of the work still presents some of the analog properties previously investigated, such as threshold voltage, drain current, body effect, sub-threshold slope and transconductance, indicating the superiority of the stacked nanowires in relation to the single-gate SOI in some aspects, especially in the capacity to supply drain current, which tends to increase the technology integration, desired by the market. The work is dedicated to presenting harmonic distortion and its effects on electrical circuits and systems, indicating that the phenomenon has great importance in several areas, being influential both in a single amplifier and in more complex electrical installations and industrial equipment. In order to obtain this parameter in the object of interest, the integral function method (IFM) was used, which allows data to be acquired only with drain current versus gate voltage curve ( $\mathrm{I}_{\mathrm{DS}} \mathrm{x} \mathrm{V}_{\mathrm{GS}}$ ), eliminating the need for alternate current (AC) measurements, which can bring more difficult noise to be dissociated from the actual signal given the magnitude of the current of the nanometric device. The results demonstrate that the harmonic distortion of the stacked nanowires is mainly related to the second order harmonic (HD2), whose value is $30 \mathrm{~dB}$ above from the third harmonic. The presented data indicate that the nanowires with higher $\mathrm{W}_{\text {FIN }}$ had major non-linearity and lower open loop voltage gain: in relation to the set of transistors with fixed length $L=100 \mathrm{~nm}$, the greatest harmonic distortion occurred for the component with $\mathrm{W}_{\mathrm{FIN}}=40 \mathrm{~nm}$, which presented total harmonic distortion $\mathrm{THD} \approx-19 \mathrm{~dB}$ and open loop voltage gain $\mathrm{Av} \approx 38 \mathrm{~dB}$ for higher $\mathrm{g}_{\mathrm{m}} / \mathrm{I}_{\mathrm{DS}}$ values, operating in moderate inversion. For the nanowire transistors with fixed width of $\mathrm{W}_{\mathrm{FIN}}=10 \mathrm{~nm}$, it was possible to observe that the major non-linearity occurs in transistors with greater lengths: for stacked nanowires of $\mathrm{L}=400 \mathrm{~nm}, \mathrm{THD} \approx-19 \mathrm{~dB}$ was obtained on higher $\mathrm{g}_{\mathrm{m}} / \mathrm{I}_{\mathrm{DS}}$ values. The relation obtained between the physical dimensions of the nanowires
\end{abstract}


transistors and the harmonic distortion are justified through the presented equations, being the correlation between the highest THD results for nanowires with higher $\mathrm{W}_{\text {FIN }}$ also explained by the greater variation of $\mathrm{dg}_{\mathrm{m}} / \mathrm{dV}_{\mathrm{GT}}$, in accordance with literature results observed for single-level nanowires. At the end of the work, new analyzes are carried out for a new set of data formed by nanowires with multiple fins. The higher magnitudes of drain current allowed to evidence the results and confirm, with more reliability, the relationship between non-linearities and the dimensional characteristics of the nanowires, since the data represents an average of results from several devices operating in parallel.

Keywords: Vertically stacked nanowires. Harmonic distortion. Operational amplifiers. Gateall-around. Triple gate. SOI transistor. 


\section{LISTA DE ILUSTRAÇÕES}

Figura 1 - Corte transversal de um nMOSFET convencional.................................................2 24

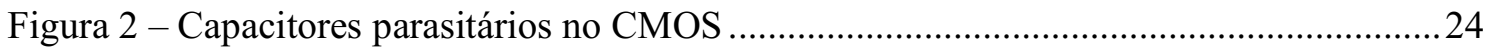

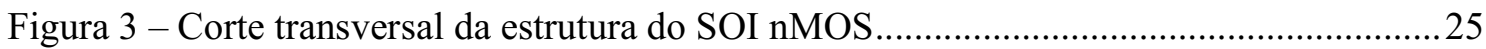

Figura 4 - Distribuição das cargas de depleção para canais longos e curtos, em MOSFETs

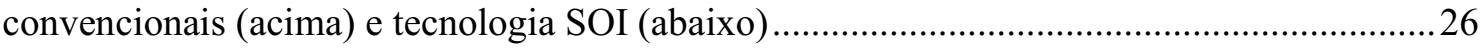

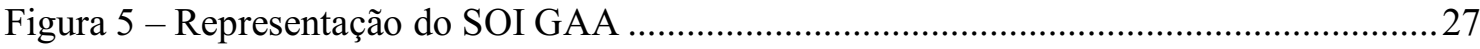

Figura 6 - Transcondutância em $\mathrm{V}_{\mathrm{DS}}=100 \mathrm{mV}$ para um SOI MOSFET convencional e um

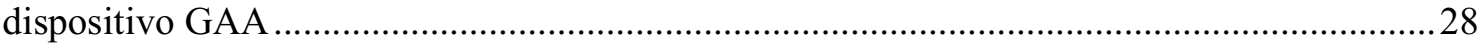

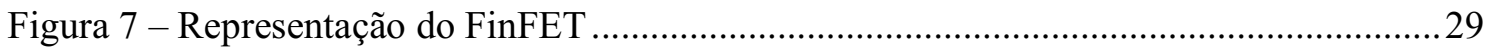

Figura 8 - Transistores de Porta Tripla (Trigates) fabricados pela Intel................................. 30

Figura 9 - Imagens TEM dos Nanofios Empilhados com diferentes larguras ( $\left.\mathrm{W}_{\text {FIN }}\right)$............. 31

Figura 10 - Tensão de Limiar versus Tensão de Substrato em dispositivos SOI .................... 35

Figura 11 - Curvas da corrente de dreno normalizada pela largura de canal do transistor, em

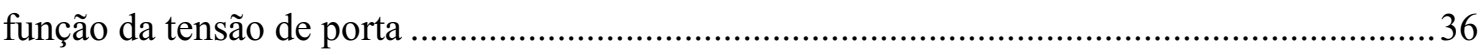

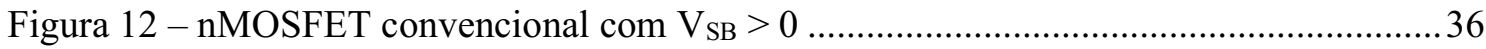

Figura 13 - Curva de corrente de dreno em função da polarização de porta em escala logarítmica

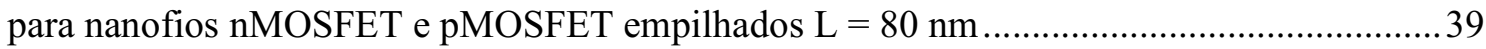

Figura 14 - Medições de mobilidade de elétrons em NW, SOI_w e SOI_n versus carga de inversão

Figura 15 - Regiões de Operação de um MOSFET genérico 42

Figura 16 - Corrente de dreno normalizada pela largura do canal em função da sobretensão de porta para transistores nanofios e SOI planares de canal largo e estreito.................................44

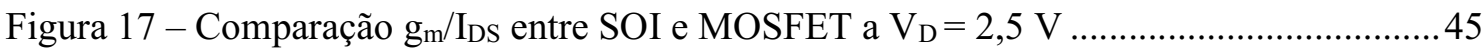

Figura $18-\mathrm{g}_{\mathrm{m}} / \mathrm{I}_{\mathrm{DS}}$ em função de $\mathrm{I}_{\mathrm{DS}} /(\mathrm{W} / \mathrm{L})$ calculado (linha) e mensurado (símbolos) para nMOS e pMOS em um FD SOI e calculado para MOS convencionais ..................................46 Figura 19 - Transcondutância versus Tensão de porta para diferentes temperaturas em dispositivos SOI e NW

Figura 20 - Amplificador de Miller com $\mathrm{C}=10 \mathrm{pF}$ e $\mathrm{f}_{\mathrm{T}}=10 \mathrm{MHz}$ para um transistor MOS (---)

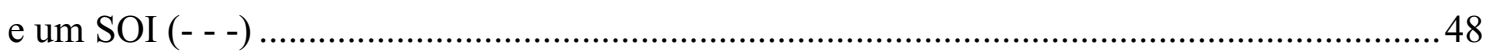

Figura 21 - Frequência fundamental e seus harmônicos..................................................... 49

Figura 22 - Senóide mensurada no domínio do tempo e da frequência...................................50 
Figura 23 - a) Fundamental e $4^{\circ}$ Harmônico. b) Fundamental e $5^{\circ}$ Harmônico. c) Somatória dos sinais de a). d) Somatórias dos sinais de b)

Figura 24 - Circuito de um Inversor de Frequência e o comportamento dos sinais em suas diferentes etapas .53

Figura 25 - Representação do efeito pelicular e sua relação com a frequência do sinal..........54

Figura 26 - Processo de normalização da curva DC para o IFM ..............................................58

Figura 27 - Aplicação da senóide na curva $\mathrm{I}_{\mathrm{DS}} \mathrm{x} \mathrm{V}_{\mathrm{GS}}$ de um transistor 59

Figura 28 - Resposta típica de THD em função da tensão de polarização, obtida no MathCAD para um nanofio empilhado .59

Figura 29 - Imagens TEM de Nanofios Empilhados com $\mathrm{W}_{\text {FIN }}=10 \mathrm{~nm}$ e $\mathrm{W}_{\text {FIN }}=5 \mathrm{~nm} \ldots \ldots . .60$

Figura 30 - IDS versus $V_{\text {GT }}$ para $\mathrm{W}$ variável com L=100 nm @ $750 \mathrm{mV}$.............................61

Figura 31 - IDs versus $V_{\text {GT }}$ para L variável com $\mathrm{W}_{\mathrm{FIN}}=10 \mathrm{~nm} @ 750 \mathrm{mV}$............................62

Figura 32 - Segunda derivada de $I_{D} x V_{G}$ de um transistor genérico .......................................62

Figura 33 - Tensão $V_{T H}$ e Inclinação de Sublimiar S em função da variação de $W_{\text {FIN }}$ (a) e L (b) .64

Figura 34 - IDS em função de $\mathrm{V}_{\mathrm{DS}}$ para $\mathrm{W}_{\text {FIN }}$ variável e L variável em diferentes $\mathrm{V}_{\mathrm{GT}}$...........65

Figura $35-\mu_{0}$ e $\theta$ em função de $\mathrm{W}_{\mathrm{FIN}}$ variável com $\mathrm{L}=100$ nm @ $\mathrm{V}_{\mathrm{DS}}=25 \mathrm{mV}$................66 Figura 36 - Resultados de THD e HD3 em função de $V_{\text {GT }}$ para $W_{\text {FIN }}$ variável com L = $100 \mathrm{~nm}$ (a) $\mathrm{V}_{\mathrm{DS}}=750 \mathrm{mV}$ .68

Figura 37 - Resultados de THD e HD3 em função de V VT para L variável com W=10 nm @ $\mathrm{V}_{\mathrm{DS}}=750 \mathrm{mV}$ 68

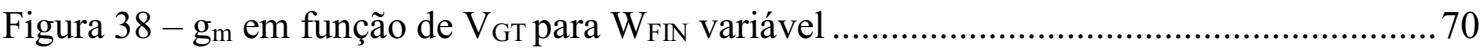

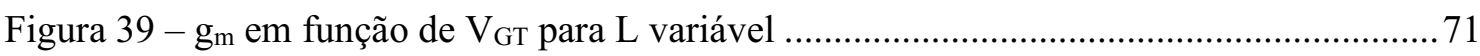

Figura 40 - Resultados de THD e HD3 em função de $g_{m} / I_{D s}$ para $W_{\text {FIN }}$ variável com $L=100$ $\mathrm{nm} @ \mathrm{~V}_{\mathrm{DS}}=750 \mathrm{mV}$ 72

Figura 41 - Resultados de THD e HD3 em função de $g_{m} / I_{D s}$ para L variável com $\mathrm{W}=10 \mathrm{~nm}$ (a) $\mathrm{V}_{\mathrm{DS}}=750 \mathrm{mV}$ .73

Figura 42 - Resultados de $A_{V}$ em função de $g_{m} / I_{D S}$ para $W_{\text {FIN }}$ variável e $L=100$ nm @ $V_{\mathrm{DS}}=$ $750 \mathrm{mV}$ 74

Figura 43 - Resultados de $A_{V}$ em função de $g_{m} / I_{D S}$ para L variável e $W_{\text {FIN }}=10 \mathrm{~nm} @ V_{\mathrm{DS}}=$ $750 \mathrm{mV}$ 75

Figura 44 - Resultados de THD/Av e HD3/Av em função de $g_{m} / I_{D s}$ para $W_{\text {FIN }}$ variável com L $=100 \mathrm{~nm} @ \mathrm{~V}_{\mathrm{DS}}=750 \mathrm{mV}$ 
Figura 45 - Resultados de THD/Av e HD3/Av em função de $\mathrm{g}_{\mathrm{m}} / \mathrm{I}_{\mathrm{DS}}$ para $\mathrm{L}$ variável com $\mathrm{W}=$

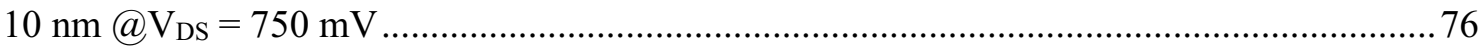

Figura 46 - Resultados de $d g_{m} / d_{\text {GT }}$ em função de $g_{m} / I_{D S}$ para $W_{\text {FIN }}$ variados dos nanofios

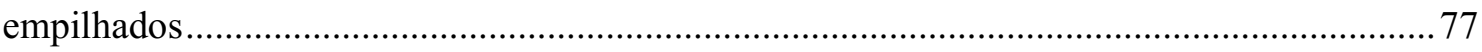

Figura 47 - IDS em função de $\mathrm{V}_{\mathrm{GT}}$ para nanofios empilhados multifins com $\mathrm{W}_{\mathrm{FIN}}$ variados @

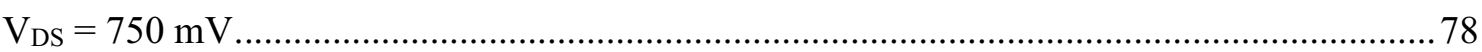

Figura $48-\mathrm{g}_{\mathrm{m}}$ em função de $\mathrm{V}_{\mathrm{GT}}$ para nanofios empilhados multifins com $\mathrm{W}_{\text {FIN }}$ variados @ $\mathrm{V}_{\mathrm{DS}}=750 \mathrm{mV}$ 79

Figura 49 - THD e HD3 em função de $\mathrm{V}_{\mathrm{GT}}$ para $\mathrm{W}_{\text {FIN }}$ variável de multifins com L =100 nm @ $\mathrm{V}_{\mathrm{DS}}=750 \mathrm{mV}$

Figura 50 - THD e HD3 em função de $\mathrm{V}_{\mathrm{GT}}$ para L variável e $\mathrm{W}_{\mathrm{FIN}}=10 \mathrm{~nm} @ \mathrm{~V}_{\mathrm{DS}}=750 \mathrm{mV}$ 81

Figura 51 - THD e HD3 em função de $g_{m} / I_{D S}$ para $W_{\text {FIN }}$ variável de multifins com $\mathrm{L}=100 \mathrm{~nm}$ (a) $\mathrm{V}_{\mathrm{DS}}=750 \mathrm{mV}$ 82

Figura 52 - THD e HD3 em função de $g_{m} / I_{D S}$ para L variável e $W_{\text {FIN }}=10$ nm @ $V_{D S}=750$ mV .83

Figura 53 - Av em função de $g_{m} / I_{D s}$ para $W_{\text {FIN }}$ variáveis com L = 100 nm @ $V_{G T}=100 \mathrm{~nm} 84$ Figura 54 - THD/Av e HD3/Av em função de $\mathrm{g}_{\mathrm{m}} / \mathrm{I}_{\mathrm{DS}}$ para $\mathrm{W}_{\text {FIN }}$ variável com L =100 nm @ V $\mathrm{V}_{\mathrm{DS}}$ $=750 \mathrm{mV}$ .84

Figura 55 - Resultados de $\mathrm{dg}_{\mathrm{m}} / \mathrm{dV}_{\mathrm{GT}}$ em função de $\mathrm{g}_{\mathrm{m}} / \mathrm{I}_{\mathrm{DS}}$ para multifins com $\mathrm{W}_{\mathrm{FIN}}$ variados 85 


\section{LISTA DE TABELAS}

Tabela 1 - $\mathrm{V}_{\mathrm{TH}} \mathrm{e} \mathrm{S}$ para diferentes $\mathrm{W}_{\mathrm{FIN}}$ com L $=100 \mathrm{~nm} @ \mathrm{~V}_{\mathrm{DS}}=25 \mathrm{mV}$...........................63

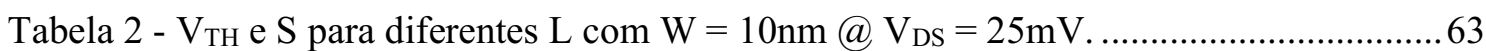

Tabela 3 - Resultados de $\mu_{0}, \theta$ e $\mathrm{R}_{\mathrm{S}} \mathrm{W}$ para diferentes $\mathrm{W}_{\mathrm{FIN}}$ de nanofios empilhados. ............67

Tabela 4 - Resultados experimentais de $g_{m}$ e $g_{D}$ em função de $V_{G T}$ para $W_{\text {Fin }}$ e L variáveis... 71

Tabela 5 - Resultados de $\mathrm{V}_{\mathrm{GT}}, \mathrm{g}_{\mathrm{m}} / \mathrm{I}_{\mathrm{DS}}$ e Av para os diferentes $\mathrm{W}_{\mathrm{FIN}}$ dos nanofios empilhados.

Tabela 6 - Resultados de $\mathrm{V}_{\mathrm{GT}}, \mathrm{g}_{\mathrm{m}} / \mathrm{I}_{\mathrm{DS}}$ e Av para os diferentes L dos nanofios empilhados..... 74 


\section{LISTA DE ABREVIATURAS E SIGLAS}

$\begin{array}{ll}\text { CMOS } & \text { Complementary Metal-Oxide-Semiconductor } \\ \text { FD SOI } & \text { Fully Depleted Silicon-On-Insulator } \\ \text { FFT } & \text { Fast Fourier Transformer } \\ \text { FINFET } & \text { Fin Field Effect Transistor } \\ \text { GAA } & \text { Gate-all-around } \\ \text { IFM } & \text { Integral Function Method } \\ \text { MOSFET } & \text { Metal Oxide Field Effect Transistor } \\ \text { NW } & \text { Nanowire } \\ \text { PD SOI } & \text { Partially Depleted Silicon-On-Insulator } \\ \text { RF } & \text { Radio Frequency } \\ \text { SOI } & \text { Silicon-On-Insulator } \\ \text { TEM } & \text { Transmission electron microscopy }\end{array}$




\section{LISTA DE SÍMBOLOS}

ao Amplitude do nível DC da série de Fourier

Av Ganho de tensão em malha aberta

$[\mathrm{dB}]$

an Amplitude do enésimo termo da série de Fourier (cosseno $\omega \mathrm{t}$ )

bn Amplitude do enésimo termo da série de Fourier (seno $\omega \mathrm{t}$ )

CDsub Capacitância entre dreno e substrato por unidade de área

CGD Capacitância entre porta e dreno por unidade de área

CGS Capacitância entre porta e fonte por unidade de área

CGsub Capacitância entre porta e substrato por unidade de área

CSsub Capacitância entre fonte e substrato por unidade de área

Cox Capacitância do óxido de porta do MOSFET por unidade de área

Cox1 Capacitância do óxido de porta da $1^{\mathrm{a}}$ interface por unidade de área

Cox2 Capacitância do óxido enterrado da $2^{\mathrm{a}}$ interface por unidade de área

D Função integral para o cálculo de distorção harmônica no IFM

gD Condutância de saída

gm Transcondutância

$\mathbf{g}_{\mathbf{m}} / \mathbf{I} \mathbf{D s}$ Relação entre a transcondutância e corrente de dreno do dispositivo

HD2 Distorção harmônica de segunda ordem

$\left[\mathrm{F} / \mathrm{cm}^{2}\right]$

$\left[\mathrm{F} / \mathrm{cm}^{2}\right]$

$\left[\mathrm{F} / \mathrm{cm}^{2}\right]$

$\left[\mathrm{F} / \mathrm{cm}^{2}\right]$

$\left[\mathrm{F} / \mathrm{cm}^{2}\right]$

$\left[\mathrm{F} / \mathrm{cm}^{2}\right]$

$\left[\mathrm{F} / \mathrm{cm}^{2}\right]$

$\left[\mathrm{F} / \mathrm{cm}^{2}\right]$

HD3 Distorção harmônica de terceira ordem

HFin Altura do fin

HDn Distorção harmônica de enésima ordem

IDs Corrente de dreno

IoFF Corrente de desligamento

$\mathbf{k}$ Constante de Boltzman

Klow Coeficiente da transcondutância de baixo campo

$\left[\mathrm{A} / \mathrm{V}^{2}\right]$

L Comprimento de canal

$[\mathrm{nm}]$

n

Fator de corpo

Na Concentração de impurezas aceitadoras de elétrons

$\left[\mathrm{cm}^{-3}\right]$

ni Concentração intrínseca de portadores

$\left[\mathrm{cm}^{-3}\right]$

q Carga elementar do elétron

Qd1 Densidade de carga de depleção controlada pela porta

$\left[\mathrm{C} / \mathrm{cm}^{2}\right]$

Qox Densidade de carga no óxido de porta do MOSFET

$\left[\mathrm{C} / \mathrm{cm}^{2}\right]$ 


\begin{tabular}{|c|c|c|}
\hline Qox1 & Densidade de carga no óxido de porta da $1^{\mathrm{a}}$ interface & {$\left[\mathrm{C} / \mathrm{cm}^{2}\right]$} \\
\hline Qox2 & Densidade de carga no óxido enterrado da $2^{\mathrm{a}}$ interface & {$\left[\mathrm{C} / \mathrm{cm}^{2}\right]$} \\
\hline Qdepl & Densidade de carga de depleção no silício & {$\left[\mathrm{C} / \mathrm{cm}^{2}\right]$} \\
\hline Qin1 & Densidade de carga de inversão na $1^{\mathrm{a}}$ interface & {$\left[\mathrm{C} / \mathrm{cm}^{2}\right]$} \\
\hline Qs2 & Densidade de carga de inversão ou acumulação na $2^{\mathrm{a}}$ interface & {$\left[\mathrm{C} / \mathrm{cm}^{2}\right]$} \\
\hline Rs & Resistência série & {$[\Omega]$} \\
\hline $\mathbf{S}$ & Inclinação de Sublimiar & [mV/déc] \\
\hline $\mathbf{T}$ & Temperatura absoluta & {$[\mathrm{K}]$} \\
\hline THD & Distorção harmônica total & {$[\mathrm{dB}]$} \\
\hline tsi & Espessura da camada de silício & {$[\mathrm{nm}]$} \\
\hline $\mathbf{V}_{\mathbf{A}}$ & Amplitude da senóide utilizada no IFM & {$[\mathrm{V}]$} \\
\hline $\mathbf{V}_{\mathbf{B}}$ & Tensão de corpo & {$[\mathrm{V}]$} \\
\hline VDS & Tensão de dreno & {$[\mathrm{V}]$} \\
\hline VEA & Tensão Early & {$[\mathrm{V}]$} \\
\hline$V_{\text {FB }}$ & Tensão de faixa plana & {$[\mathrm{V}]$} \\
\hline $\mathbf{V}_{\mathbf{G 1}}$ & Tensão aplicada à porta (relacionado à $1^{\mathrm{a}}$ interface do SOI) & {$[\mathrm{V}]$} \\
\hline $\mathbf{V}_{\mathrm{G} 2}$ & Tensão aplicada ao back-gate (relacionado à $2^{\mathrm{a}}$ interface do SOI) & {$[\mathrm{V}]$} \\
\hline $\mathbf{V}_{\text {GS }}$ & Tensão de porta & {$[\mathrm{V}]$} \\
\hline $\mathbf{V}_{\text {GT }}$ & Sobretensão de condução & {$[\mathrm{V}]$} \\
\hline $\mathbf{V}_{\mathbf{s}}$ & Tensão de fonte & {$[\mathrm{V}]$} \\
\hline $\mathbf{V}_{\text {TH }}$ & Tensão de limiar & {$[\mathrm{V}]$} \\
\hline $\mathbf{V}_{\text {TH1, acc2 }}$ & Tensão de limiar para $2^{\mathrm{a}}$ interface em regime de acumulação & {$[\mathrm{V}]$} \\
\hline $\mathbf{V}_{\text {TH1,inv2 }}$ & Tensão de limiar para $2^{\mathrm{a}}$ interface em regime de inversão & {$[\mathrm{V}]$} \\
\hline$V_{\text {TH1,depl2 }}$ & Tensão de limiar para $2^{\mathrm{a}}$ interface em regime de depleção & {$[\mathrm{V}]$} \\
\hline $\mathbf{W}$ & Largura de canal & {$[\mathrm{nm}]$} \\
\hline $\mathbf{W}_{\text {FIN }}$ & Largura do fin & {$[\mathrm{nm}]$} \\
\hline $\mathbf{X}_{\mathbf{C}}$ & Reatância capacitiva & {$[\Omega]$} \\
\hline $\mathbf{X}_{\text {dmáx }}$ & Máxima largura da região de depleção & {$[\mathrm{nm}]$} \\
\hline $\mathbf{X}_{\mathbf{L}}$ & Reatância indutiva & {$[\Omega]$} \\
\hline$\alpha$ & Parâmetro resultante da associação de capacitâncias do transistor & - \\
\hline$\varepsilon_{\mathrm{Si}}$ & Permissividade do silício & {$\left[\mathrm{F} / \mathrm{cm}^{2}\right]$} \\
\hline$\Phi_{\mathbf{F}}$ & Potencial de Fermi & {$[\mathrm{V}]$} \\
\hline $\boldsymbol{\Phi}_{M S}$ & Diferença de função trabalho entre metal e silício & {$[\mathrm{V}]$} \\
\hline
\end{tabular}




$\begin{array}{llr}\text { Фs1 } & \text { Potencial de superfície da } 1^{\text {a }} \text { interface } & {[\mathrm{V}]} \\ \boldsymbol{\Phi}_{\mathbf{s} 2} & \text { Potencial de superfície da } 2^{\mathrm{a}} \text { interface } & {[\mathrm{V}]} \\ \boldsymbol{\gamma} & \text { Constante de corpo } & {\left[\mathrm{V}^{1 / 2}\right]} \\ \boldsymbol{\mu} & \text { Mobilidade } & {\left[\mathrm{cm}^{2} / \mathrm{V} . \mathrm{s}\right]} \\ \boldsymbol{\mu}_{\mathbf{0}} & \text { Mobilidade de baixo campo } & {\left[\mathrm{cm}^{2} / \mathrm{V} . \mathrm{s}\right]} \\ \boldsymbol{\mu}_{\text {eff }} & \text { Mobilidade efetiva } & {\left[\mathrm{cm}^{2} / \mathrm{V} . \mathrm{s}\right]} \\ \boldsymbol{\mu}_{\mathbf{m a x}} & \text { Mobilidade máxima } & {\left[\mathrm{cm}^{2} / \mathrm{V} . \mathrm{s}\right]} \\ \boldsymbol{\mu}_{\mathbf{m i n}} & \text { Mobilidade mínima } & {\left[\mathrm{cm}^{2} / \mathrm{V} . \mathrm{s}\right]} \\ \boldsymbol{\mu}_{\text {sii }} & \text { Mobilidade de espalhamento por impurezas ionizadas } & {\left[\mathrm{cm}^{2} / \mathrm{V} . \mathrm{s}\right]} \\ \boldsymbol{\Theta} & \text { Coeficiente de degradação de mobilidade } & {\left[\mathrm{V}^{-1}\right]}\end{array}$




\section{SUMÁRIO}

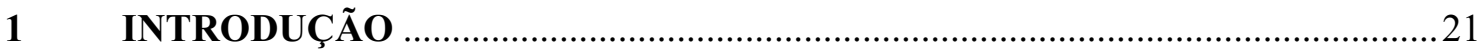

2 TRANSISTORES MOS E PARÂMETROS ELÉTRICOS …..............................22

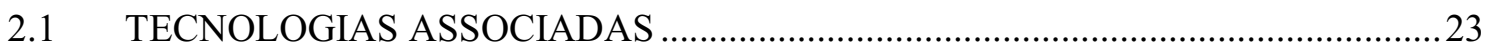

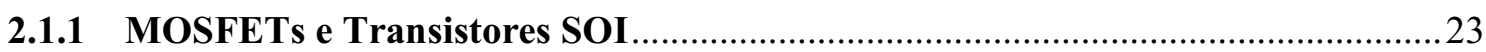

2.1.2 Transistor MOS com Porta Circundante Gate All Around .................................27

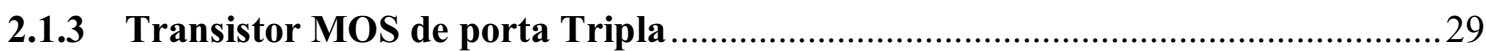

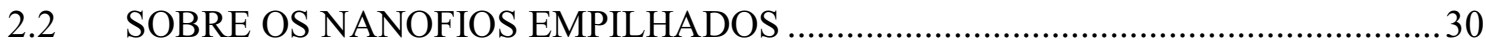

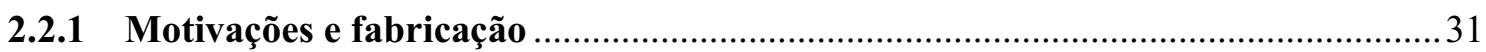

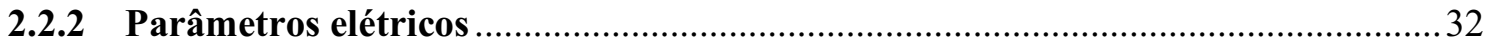

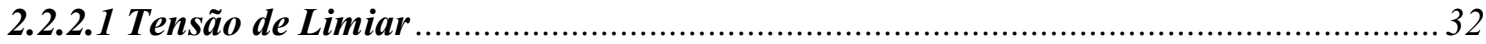

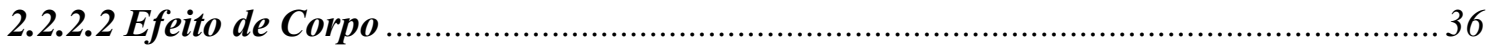

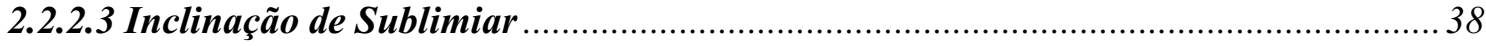

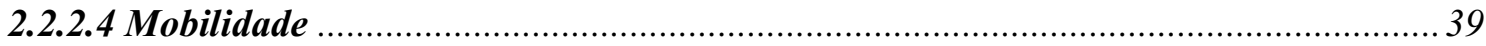

2.2.2.5 Operação em Triodo e Saturação e Corrente de dreno I Is........................................ 42

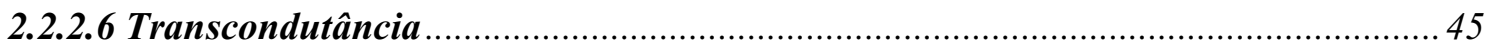

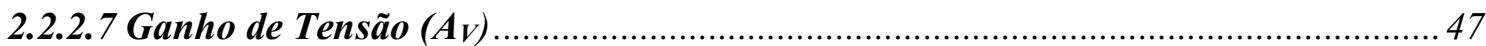

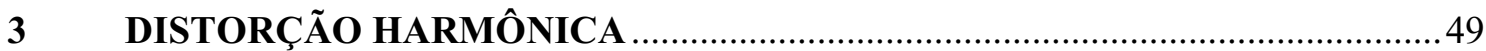

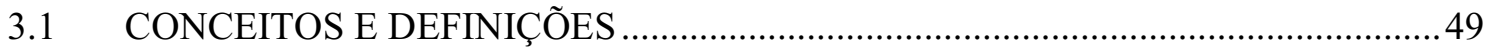

3.2 ORIGENS E CONSEQUÊNCIAS DA DISTORÇÃO HARMÔNICA.......................52

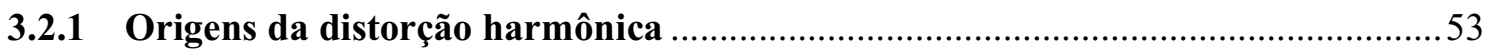

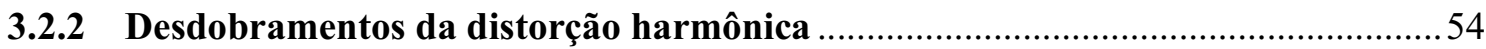

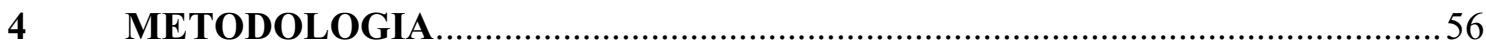

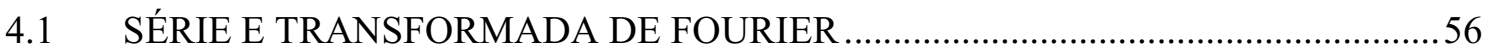

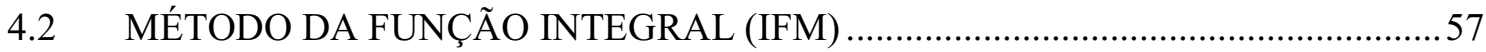

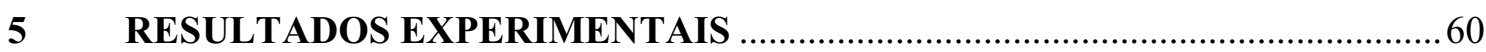

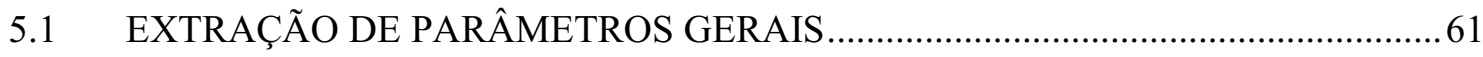

5.1.1 Corrente de dreno em função da sobretensão de condução ……..........................61

5.1.2 Tensão de Limiar (VTH) e Inclinação de Sublimiar (S) ......................................62 


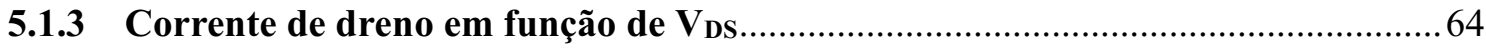

5.1.4 Mobilidade de baixo campo e Degradação da Mobilidade ...................................66

5.2 RESULTADOS DE NÃO LINEARIDADE PARA OS TRANSISTORES NANOFIOS

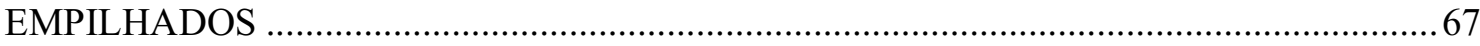

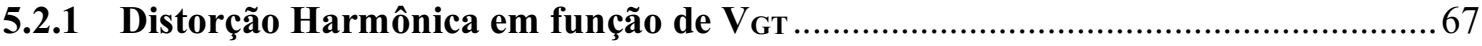

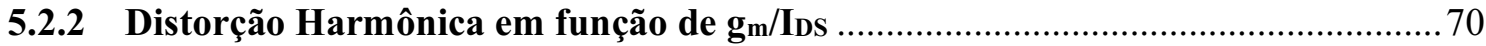

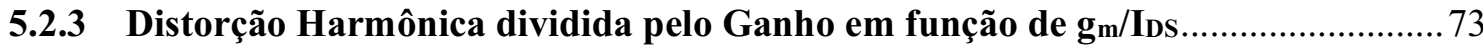

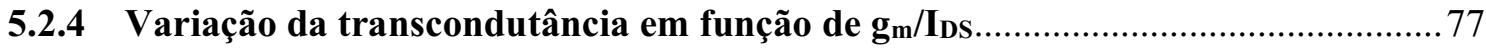

5.3 NANOFIOS EMPILHADOS MULTIFINS E DE COMPRIMENTOS VARIADOS .78

5.3.1 Corrente e Transcondutância dos Multifins.......................................................... 78

5.3.2 Distorção Harmônica em função de $V_{G T}$ para os Nanofios Empilhados Multifins e

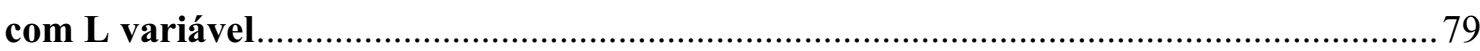

5.3.3 Distorção Harmônica em função de $\mathbf{g}_{\mathrm{m}}$ /Ios para os Nanofios Empilhados Multifins

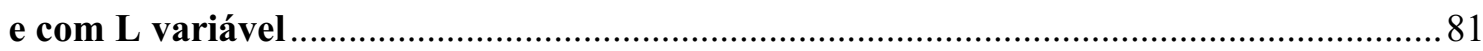

5.3.4 Distorção Harmônica dividida pelo Ganho em função de $g_{\mathrm{m}} / \mathrm{IDs}_{\text {para }}$ os Nanofios

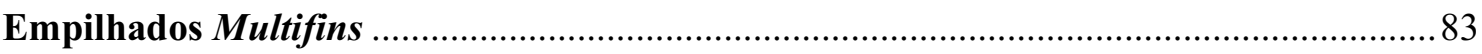

5.3.5 Variação da transcondutância em função de $\mathbf{g}_{\mathrm{m}} / \mathrm{I}_{\mathrm{DS}}$ para os Multifins................. 85

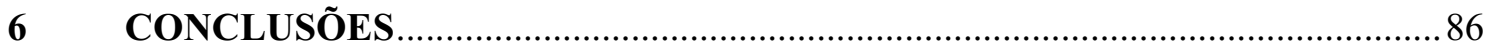

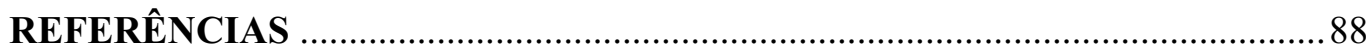

ANEXO A - FLUXOGRAMA DO PROCESSO DO MÉTODO DA FUNÇÃO

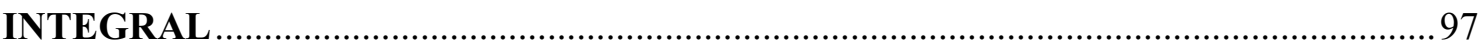

ANEXO B - ARTIGO CIENTÍFICO PUBLICADO NA JOURNAL OF

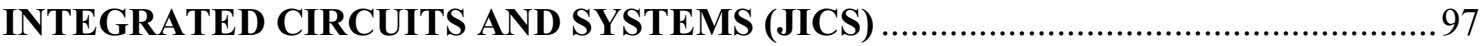




\section{INTRODUÇÃO}

Existe uma enorme gama de pesquisas dedicadas ao estudo de novos dispositivos eletrônicos e novos circuitos voltados às melhorias no desempenho de aplicações essencialmente digitais. Menor tempo de resposta, maior velocidade de transmissão dados e processadores mais potentes estão entre os tópicos mais estudados (JOHANSSON; WANHAMMAR, 2000), (GUPTA et al., 2019), (RAI; CHAUDHURI, 2017). Entretanto, fato é que o mundo possui uma natureza de fenômenos analógicos e, portanto, o contínuo avanço no desempenho de componentes e circuitos mistos, analógico-digitais, se torna igualmente importante, já que somente desta maneira será possível para a academia e indústria desenvolverem novos sistemas eletrônicos que captem sinais naturais e gerem sinais analógicos com maior acurácia e eficiência. Conversores digital-analógicos, transmissores de sinais de RF e amplificadores são alguns dos exemplos de circuitos amplamente utilizados e que, atualmente, integram inúmeros produtos e tecnologias, nos mais variados campos de aplicação (SONG; LUO; LI, 2012).

Embora o comportamento analógico de um dispositivo eletrônico possua diversas áreas de estudo, como sua capacidade de amplificar corrente e irradiar sinais de radiofrequência, uma das principais características desejadas em circuitos analógicos é a linearidade, a qual se caracteriza pela dependência linear entre um sinal de saída e de entrada. A manutenção dessa dependência linear entre os sinais de saída e entrada, em outras palavras, faz com que os sistemas analógicos não introduzam alterações ao sinal (SEDRA; SMITH, 2005). A existência de uma relação não linear entre os sinais de saída e entrada, decorrente do circuito analógico empregado, é denominada de não linearidade ou distorção harmônica. Esta característica fundamental independe da complexidade do circuito analógico em questão, podendo este ser composto, inclusive, por um único transistor. Esse trabalho se dedica a explorar o desempenho de um novo transistor frente a este fenômeno de grande relevância, relacionado à característica e qualidade do sinal obtido após a inserção de sinais de entrada, levando-se em consideração suas partes integrantes e suas razões físicas.

Os transistores de interesse para este trabalho são os nanofios empilhados, que possuem arquitetura inovadora e diversas outras propriedades a serem explanadas no decorrer desta dissertação. Sua forma construtiva permite poupar área de silício e, consequentemente, elevar a densidade de corrente elétrica dos transistores por lâmina, uma vez que a idealização do componente tange o princípio da fabricação vertical de transistores (DUPRÉ et al., 2008). Desta forma, uma área que outrora seria ocupada por dois transistores posicionados horizontalmente, 
por exemplo, passa a aumentar consideravelmente a sua capacidade de corrente, já que a configuração empilhada - nesta mesma área - permitiria alocar o equivalente a quatro dos mesmos transistores. Isto está alinhado com a tendência de miniaturização dos dispositivos, alavancada pela ânsia de mercado de se obter melhores e mais potentes equipamentos eletrônicos sem aumentar o tamanho físico do sistema, vide celulares de última geração e os super computadores (SANTINO, 2020). Além disso, a implementação dos nanofios empilhados em tecnologia SOI se traduz na criação de um transistor com uma série de vantagens em relação ao transistor MOS convencional, conforme será visto no próximo capítulo. Até onde se sabe, o estudo específico da distorção harmônica para este componente ainda não foi contemplado na literatura, em dissertações ou teses.

No capítulo 2, serão apresentados conceitos fundamentais, que servirão de base para o entendimento do dispositivo estudado, focando em seus aspectos construtivos e no que já se sabe referente à algumas de suas propriedades elétricas. Os parâmetros elétricos relacionados aos transistores serão conceituados, modelados matematicamente, discutidos e comparados para diversas tecnologias, enriquecendo o tema.

A definição da distorção harmônica se encontrará no capítulo 3, assim como sua explicação matemática e alguns exemplos clássicos de aplicações onde sua existência se torna bastante influente e considerável, no desempenho de circuitos e sistemas elétricos.

O capítulo 4 tem como objetivo apresentar a metodologia empregada neste estudo, que permitiu a obtenção da distorção harmônica nos inovadores transistores nanofios empilhados. O tópico servirá também como comparação crítica entre outro método mais tradicional, permeando a discussão a respeito de seus prós e contras.

Finalmente, no capítulo 5 serão apresentadas diversas curvas e resultados obtidos relacionados às características analógicas do transistor nanofio empilhado. Algumas abordagens diferentes propiciarão um olhar crítico da distorção harmônica sob alguns prismas de comparação.

Os últimos capítulos se dedicam a concluir a respeito do desempenho analógico dos nanofios empilhados, assim como apresentar o leque de artigos e documentos utilizados como fundação dos conceitos apresentados. 


\section{TRANSISTORES MOS E PARÂMETROS ELÉTRICOS}

Os componentes centrais nesta pesquisa são os nanofios transistores empilhados, fabricados no CEA-LETI, França (BARRAUD et al., 2018), e disponibilizados para o Centro Universitário FEI. Tais dispositivos são fabricados em substratos tipo silício sobre isolante SOI (Silicon-On-Insulator) e consistem de transistores MOS tridimensionais, com duas camadas empilhadas: a camada superior é formada por um transistor de porta circundante MOS tipo GAA (Gate-all-around) e a camada inferior consiste de um transistor MOS de porta tripla (ou Trigate). A seguir serão abordadas características fundamentais destas configurações e das arquiteturas de interesse. Uma segunda etapa deste capítulo apresentará alguns dos comportamentos analógicos já estudados para estes transistores.

\subsection{TECNOLOGIAS ASSOCIADAS}

Antes de explanar a respeito da composição dos nanofios empilhados, que representam um dos elementos mais inovadores na tecnologia de semicondutores (BARRAUD et al., 2018) é preciso traçar uma linha lógica e evolutiva de alguns dispositivos MOS, a fim de pautar as bases teóricas necessárias para entender com maior clareza as características e motivações de criação dos transistores utilizados nos experimentos deste trabalho.

\subsubsection{MOSFETs e Transistores SOI}

Desde o final do século passado, outras estruturas de transistores diferentes dos MOSFETs convencionais vêm sendo propostas, estudadas e fabricadas (COLINGE et al., 1990). A contínua busca pela redução nas dimensões físicas de cada componente está entre as maiores motivações que levam a este esforço científico, e a indústria tem um interesse particular neste avanço, uma vez que a integralização de mais componentes em uma mesma área de silício pode resultar em um produto com maior capacidade de armazenamento de memória e/ou melhor velocidade de processamento, ou ainda em sistemas eletrônicos com maior densidade de transistores e maior densidade de funções executadas. Porém, a diminuição do comprimento de canal dos transistores MOS gera efeitos colaterais indesejados, que prejudicam a funcionalidade destes dispositivos. Dentre eles, podem-se citar os efeitos de canal curto, como a diminuição da tensão de limiar $\left(\mathrm{V}_{\mathrm{TH}}\right)$, o aumento da corrente elétrica quando o transistor está desligado (I IFF), o efeito punch-through, a degradação da inclinação de sublimiar, entre outros 
(COLINGE, 2004). Além disso, o transistor MOS convencional possui diversas capacitâncias parasitárias, inerentes à sua arquitetura (RICCO; VERSARI; ESSENI, 1995), que afetam consideravelmente o seu tempo de resposta em meio aos circuitos mistos e digitais. A Figura 1 representa a forma construtiva do nMOSFET convencional, enquanto a Figura 2 indica seus capacitores parasitas, onde $\mathrm{C}_{\text {Ssub }}, \mathrm{C}_{\text {Dsub }}$ e $\mathrm{C}_{\text {Gsub }}$ representam as capacitâncias entre fonte e substrato, dreno e substrato, e porta e substrato, respectivamente, enquanto $\mathrm{C}_{\mathrm{GS}}$ e $\mathrm{C}_{\mathrm{GD}}$ são as capacitâncias entre porta e fonte e entre porta e dreno.

Figura 1 - Corte transversal de um nMOSFET convencional

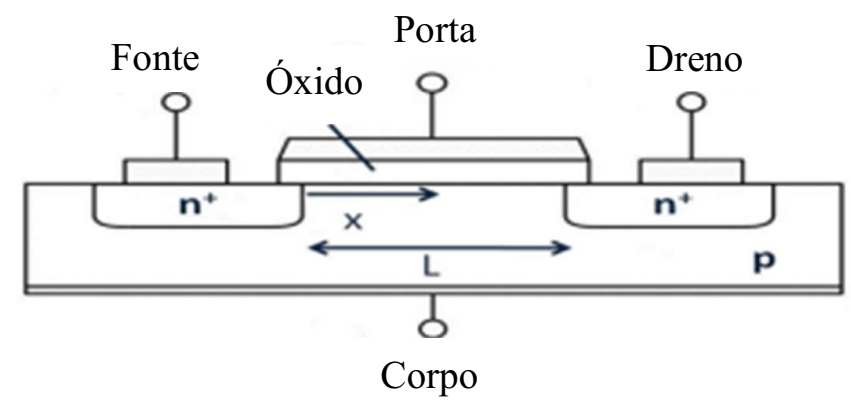

Fonte: Autor "adaptado de" Agarwal, 2020

Figura 2 - Capacitores parasitários no CMOS

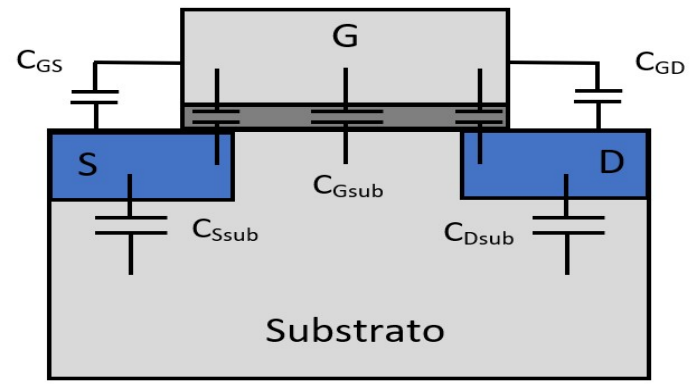

Fonte: Autor

Na década 1960, porém, surgia uma das tecnologias alternativas mais promissoras, a tecnologia SOI (MANASEVIT; SIMPSON, 1964). A ilustração do dispositivo pode ser encontrada na Figura 3. A presença de uma camada isolante entre as regiões ativas e o substrato de silício reduziu consideravelmente as capacitâncias parasitárias de junção, graças à menor permissividade e maior espessura do óxido enterrado que separa as regiões. Uma menor capacitância parasitária se traduz em desempenho mais veloz (PARTRIDGE, 1986), uma vez que a constante de tempo é descrita em função da multiplicação dos elementos resistivos pelos capacitivos. Além disso, diversos outros ganhos foram obtidos em relação ao CMOS 
convencional, dentre eles: eliminação do efeito tiristor parasitário, maior resistência à elementos radioativos, menor consumo e dissipação de potência e redução do efeito de corpo (PARTRIDGE, 1986) (COLINGE, 2004). Importante pontuar ainda que o transistor SOI é denominado parcialmente depletado (PD SOI) caso a espessura de sua camada de silício seja maior do que duas vezes a largura máxima da região de depleção. Entretanto, caso o dispositivo possua uma fina camada de silício, de forma que a região de depleção se estenda da porta até à superfície do óxido enterrado, este é denominado totalmente depletado (FD SOI) (KRISHNAN et al., 1995).

Figura 3 - Corte transversal da estrutura do SOI nMOS

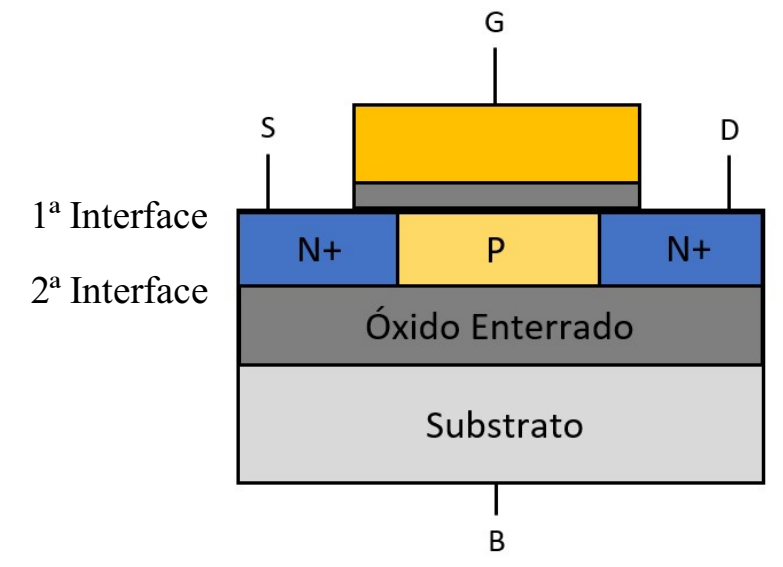

Fonte: Autor

A despeito dos avanços da tecnologia, um dos principais elementos de diferenciação do FD SOI MOSFET é, sem dúvida, a possibilidade de redução do comprimento de canal do dispositivo. Em um transistor MOS convencional, a região de depleção se estende para além do canal, acumulando-se também nas áreas inferiores das regiões de fonte e dreno. Ao reduzir as dimensões do componente, o campo elétrico desta região se mostra preponderante em relação às cargas do canal efetivamente controladas pela porta, o que causa os efeitos de canal curto. Em contrapartida, no SOI existe o óxido enterrado entre as regiões ativas e o substrato, que diminui a região de depleção entre a fonte e o substrato e entre o dreno e o substrato. Desta forma, ao reduzir as dimensões do transistor SOI, a área ocupada pela depleção é comparativamente inferior, atenuando os efeitos de canal curto e permitindo a construção de dispositivos menores. Tal comparação pode ser observada na Figura 4, que evidencia a 
proporção da área ocupada pela região de depleção das junções em ambas as tecnologias, sendo Qd1 a carga de depleção efetivamente controlada pela porta.

Figura 4 - Distribuição das cargas de depleção para canais longos e curtos em MOSFETs convencionais (acima) e tecnologia SOI (abaixo)

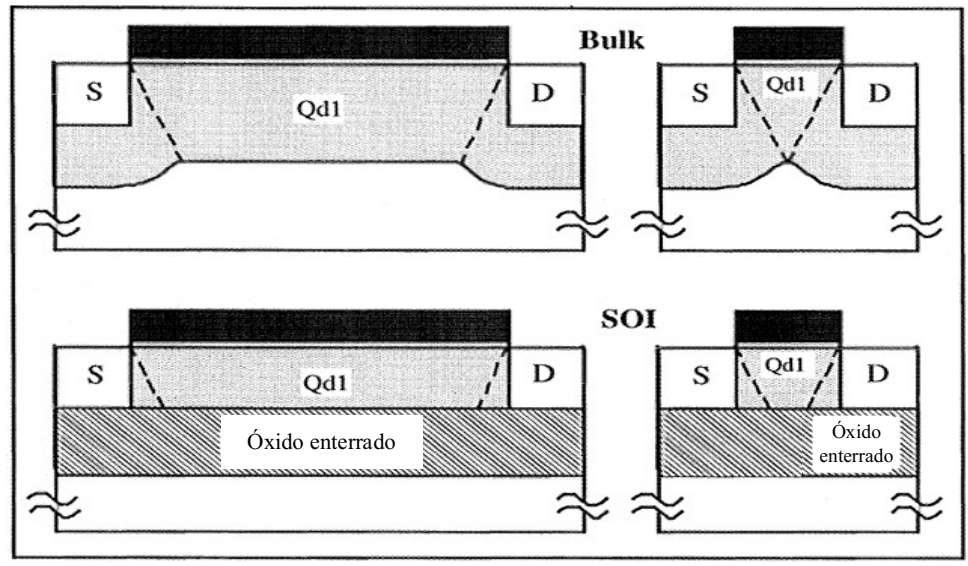

Fonte: Autor "adaptado de" Colinge, 2004

Apesar de possuir muitas vantagens, o dispositivo também apresenta seus pontos negativos. O kink effect, por exemplo, se refere à elevação abrupta de corrente que ocorre no SOI nMOSFET parcialmente depletado (PD) (PAVANELLO, 2000). As lacunas geradas a partir da ionização por impacto (após o estrangulamento do canal) se acumulam na região de corpo, elevando o potencial elétrico nesta região e diminuindo a tensão de limiar $\left(\mathrm{V}_{\mathrm{TH}}\right)$, o que aumenta abruptamente a corrente IDs. Isto resulta em um processo recursivo prejudicial, já que gera mais pares elétron-lacunas por impacto, reiniciando o processo até levar ao efeito avalanche. O acúmulo destas cargas não acontece no SOI totalmente depletado (FD SOI), uma vez que as lacunas acumuladas podem ser recombinadas mais facilmente na fonte devido à polarização direta existente entre o canal e a fonte. Utilizar SOI totalmente depletado também se mostra interessante à medida que a disposição apresenta uma alta corrente de saturação e curva de sublimiar mais inclinada e próxima do limite teórico (COLINGE, 1991). Outro desafio da tecnologia, porém, está associado ao bipolar parasitário (existente na junção fonte-canaldreno), pois o isolante faz com que a junção NPN (no caso do SOI nMOS) possua base flutuante, o que pode provocar amplificação de correntes indesejadas (single-transistor latchup), além de diminuir a tensão de ruptura no dreno do SOI (CEUSTER; FLANDRE, 1994).

Tais melhorias alcançadas nesta tecnologia serviram de motivação para o estudo e criação de outros tipos de transistores, que embora possuam outras arquiteturas, ainda mantém 
o óxido enterrado. Neste sentido, pode-se citar o "Twin-Gate" (GAO et al., 1992), o LDD (Lightly Doped Drain) (PIDIN et al., 1995), o GC SOI (Graded-Channel) (PAVANELLO; MARTINO; FLANDRE, 2001), o GAA (COLINGE et al., 1990), o Trigate (DOYLE, 2003) entre outros. Os dois últimos citados fazem parte da arquitetura dos nanofios empilhados, e serão melhor descritos a seguir.

\subsubsection{Transistor MOS com Porta Circundante Gate All Around}

O transistor MOS com porta circundante, ou GAA (Gate-All-Around) caracteriza-se por um transistor cujo metal de porta e óxido de porta envolvem totalmente a sua região de canal. A representação esquemática de um nMOSFET GAA é apresentada na Figura 5. Inicialmente, a motivação que levou ao desenvolvimento deste componente estava relacionada à pretensão de se obter um transistor mais resistente à radiação e com melhores desempenhos elétricos no que tange aos efeitos de canal curto e transcondutância (COLINGE et al., 1990). De fato, esses benefícios são observados no transistor GAA, e abaixo serão relacionados maiores detalhes a fim de explicar estes fenômenos.

Figura 5 - Representação do SOI GAA
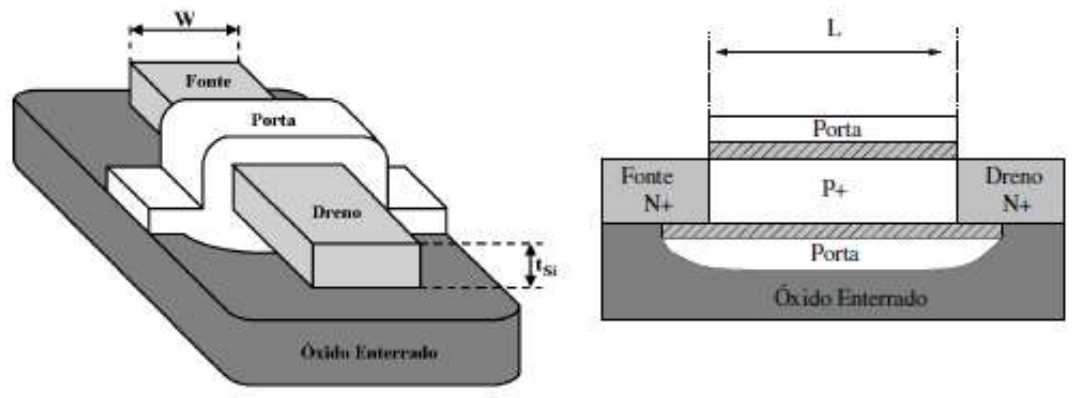

Fonte: Doria, 2010

Uma vez que a porta envolve todo o canal do dispositivo, é possível obter um melhor controle das cargas nesta região através da tensão de porta $\left(V_{G S}\right)$. Por consequência, o campo elétrico presente no canal se torna mais dependente das tensões aplicadas nos terminais do que das cargas geradas por impacto acumuladas e da região de depleção entre as junções, mesmo em dimensões menores, diferentemente do que foi visto no transistor SOI PD, por exemplo. Isso explica o porquê do transistor GAA apresentar reduções nos efeitos de canal curto e inclinação de sublimiar mais próxima do limite teórico em temperatura ambiente, $60 \mathrm{mV} /$ déc (DORIA, 2010). 
Esse transistor é equivalente a um transistor MOS de duas portas, pois o tamanho da porta lateral não gera contribuição de corrente relevante em relação ao restante do material que envolve a largura do canal, já que este último possui dimensões muito maiores. Desta maneira, a corrente IDS pode ser estimada como próxima do dobro de um FD SOI com porta única (considerando transistores com mesmo comprimento de canal, tensão de limiar, tensão de porta e concentração de dopantes), uma vez que nesta situação tem-se o dobro da área de condução efetiva de corrente, ou seja, o dobro da largura em relação ao transistor de porta única $\left(\mathrm{W}_{\mathrm{GAA}}=\right.$ 2 W PORTA ÚNICA). A partir desta constatação, é possível perceber seu ganho em termos de transcondutância $\left(\mathrm{g}_{\mathrm{m}}\right)$, já que este termo é expresso em função da variação de corrente de dreno (IDS) pela variação de tensão de porta ( $\left.\mathrm{V}_{\mathrm{GS}}\right)$, conforme eq. (1).

$$
g_{m}=\frac{\partial I_{D S}}{\partial V_{G S}}
$$

De fato, tal relação já foi demonstrada em (COLINGE, 2004), que relatou a superioridade da transcondutância de um GAA em relação a um SOI de porta única, conforme mostra a Figura 6. Na prática, o desempenho do GAA é até melhor do que o dobro de um SOI de porta única (representado pela curva “SOI (x2)"), uma vez que no gate-all-around com fina camada de silício existe o fenômeno da inversão de volume (BALESTRA et al., 1987), onde os portadores acabam por se concentrar na região mais central do silício, ao invés de se aproximarem das regiões de interface (silício/óxido de porta).

Figura 6 - Transcondutância em $\mathrm{V}_{\mathrm{DS}}=100 \mathrm{mV}$ para um SOI MOSFET convencional e um dispositivo GAA

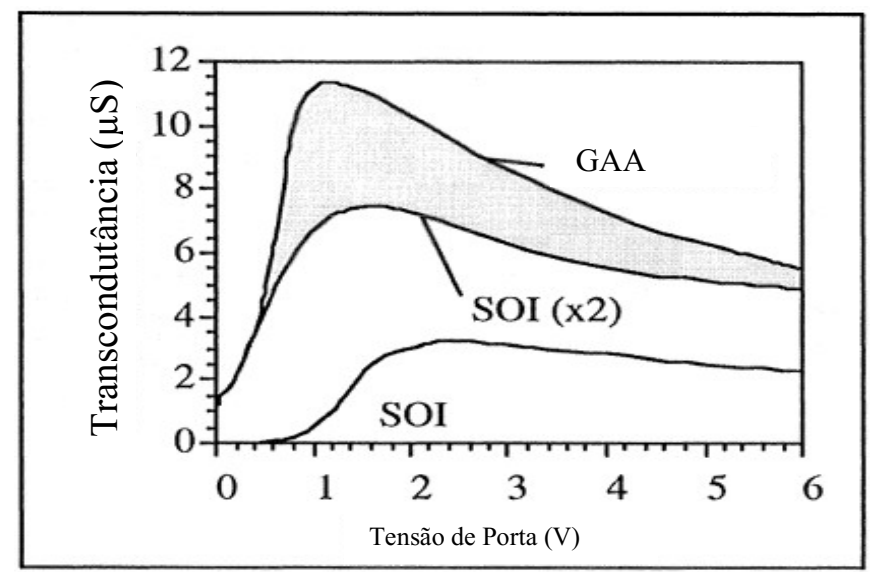

Fonte: Autor "adaptado de" Colinge, 2004 


\subsubsection{Transistor MOS de porta Tripla}

O transistor MOS de porta tripla (ou Trigate) também é conhecido como FinFET de porta tripla, e seu nome revela uma de suas peculiaridades: nesta tecnologia, as regiões de fonte, canal e dreno são construídas de forma a manter uma estrutura vertical, denominada "fin". Uma vez possuindo altura e largura de fin com dimensões próximas $\left(\mathrm{W}_{\mathrm{FIN}}=\mathrm{H}_{\mathrm{FIN}}\right)$, a área encoberta pelo eletrodo de porta se torna semelhante em todas as faces do dispositivo, confirmando sua natureza de porta tripla. A Figura 7 demonstra a representação do FinFET de porta tripla. Embora sua denominação possa sugerir independência entre as portas, estas então interconectadas e atuam como um único eletrodo de porta, que controla a condução em três faces da aleta de silício, duas em suas laterais e uma em seu topo.

Os transistores Trigate também têm melhores desempenhos elétricos em relação ao transistor SOI MOS convencional (COLINGE, 2004). A mesma lógica explicada no item anterior, referente à diminuição dos efeitos de canal curto e curva de sublimiar se aplica, já que três portas atuando nas cargas do canal se traduz em melhor controle da corrente do transistor, mesmo em dispositivos de menores dimensões. Além disso, é possível perceber que essa tecnologia é uma ótima alternativa a ser empregada em circuitos amplificadores de corrente, pois nesta configuração IDs possui três vezes mais área de canal para conduzir, o que explica sua corrente de dreno ser estimada como o triplo da corrente de um SOI de porta única para transistores cuja largura e altura do fin sejam iguais, e sua maior transcondutância (DORIA, 2010).

Figura 7 - Representação do FinFET

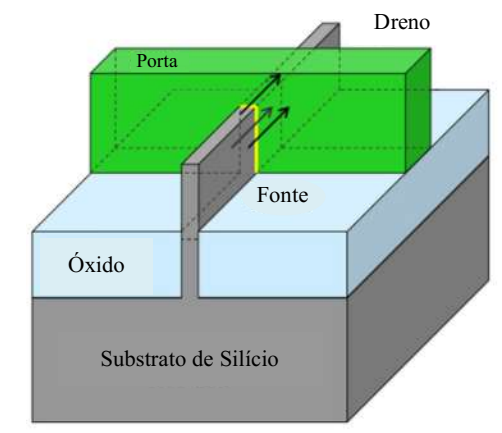

Fonte: Autor “adaptado de” Brant, 2016

Não é incomum aproveitar as mesmas estruturas de porta, fonte e dreno na fabricação de diversos FinFETs, com o intuito de se obter maiores correntes de dreno (LU; LEE; 
WACHNIK, 2015). Os chamados "multi-fingers" se caracterizam por diversos transistores controlados pela mesma porta, onde cada fin se encontra paralelo um ao outro, mas interconectados em sua fonte e dreno. Ao aplicar tensão de porta $\mathrm{V}_{\mathrm{GS}}$ acima da tensão de limiar $\mathrm{V}_{\mathrm{TH}}$, as diferentes correntes fluindo pelos diversos transistores se acumulam, gerando maiores ganhos de corrente de dreno. Em chips industriais, a lâmina de silício também é aproveitada para criar várias "colunas" de transistores, onde o dreno de um Trigate se torna a fonte do componente adjacente e assim por diante, maximizando sua amplificação. A Figura 8 mostra a fotografia de um conjunto de transistores de porta tripla fabricados pela Intel, desenvolvidos em 2011 (BRANT, 2016). Retomando a questão da densidade de integração de componentes, ao observar os multi-fingers da Figura 8, fica claro perceber o ganho trazido pela proposta de empilhamento de transistores, uma vez que caso o chip fosse ocupado por dispositivos verticalmente empilhados, seria possível reduzir a dimensão do produto pela metade (aproximadamente), graças à diminuição de área horizontal ocupada por transistores.

Figura 8 - Transistores de Porta Tripla

(Trigates) fabricados pela Intel

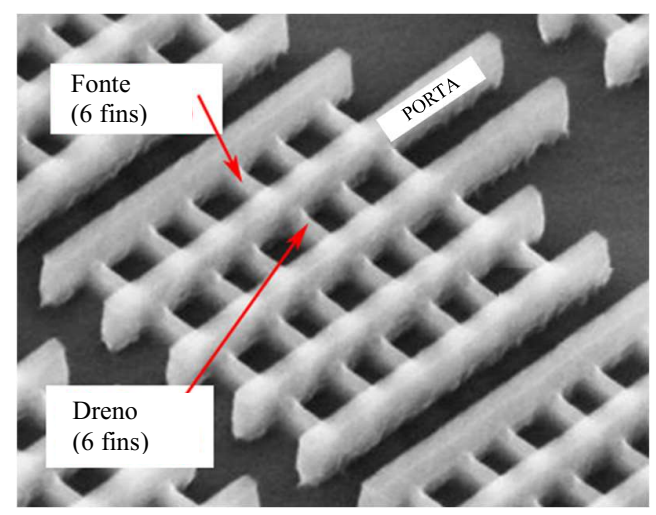

Fonte: Autor "adaptado de" Brant, 2016

\subsection{SOBRE OS NANOFIOS EMPILHADOS}

Uma vez apresentadas as tecnologias e características dos transistores que compõem os nanofios empilhados, serão abordados maiores detalhes da nova estrutura de interesse deste trabalho. Esta seção também será aproveitada para comentar os parâmetros elétricos especialmente relevantes do componente, que serão utilizados nas análises e resultados posteriores. 


\subsubsection{Motivações e fabricação}

Como visto anteriormente, tanto o transistor GAA quanto o transistor Trigate possuem desempenhos excepcionalmente superiores em relação aos transistores SOI convencionais. Seus ganhos de corrente foram relacionados às suas formas construtivas, que possuem mais faces para controle de porta e condução de corrente no canal. Desta forma, é natural prever que um único transistor composto pela união de ambos os dispositivos apresente propriedades ainda mais atrativas, do ponto de vista da amplificação de IDs. Ademais, a proposta de se empilhar níveis de transistores (ERNST et al., 2006) aumenta substancialmente a capacidade de integração da tecnologia, característica essencialmente vital para o contínuo avanço da eletrônica e desenvolvimento de produtos cada vez mais poderosos em processamento e memória, e menores, anseio da indústria e da comunidade científica, assim como já citado na seção 2.1.1.

Em vista destas motivações, a CEA-LETI, que é um dos maiores laboratórios de nanotecnologia do mundo, fabricou os primeiros chips contendo os nanofios empilhados. Sua fabricação foi primeiramente relatada na referência (DUPRE, 2008). A Figura 9 traz uma fotografia de dois transistores finalizados, com diferentes larguras de fin (W $\mathrm{W}_{\mathrm{FIN}}$ ), evidenciando o transistor GAA (camada superior), o transistor Trigate (camada inferior) e sua propriedade empilhada. Essa imagem foi obtida graças à técnica TEM (transmission electron microscopy), que consiste em incidir um raio de elétrons no objeto de estudo.

Figura 9 - Imagens TEM dos Nanofios Empilhados com diferentes larguras ( $\left.\mathrm{W}_{\mathrm{FIN}}\right)$
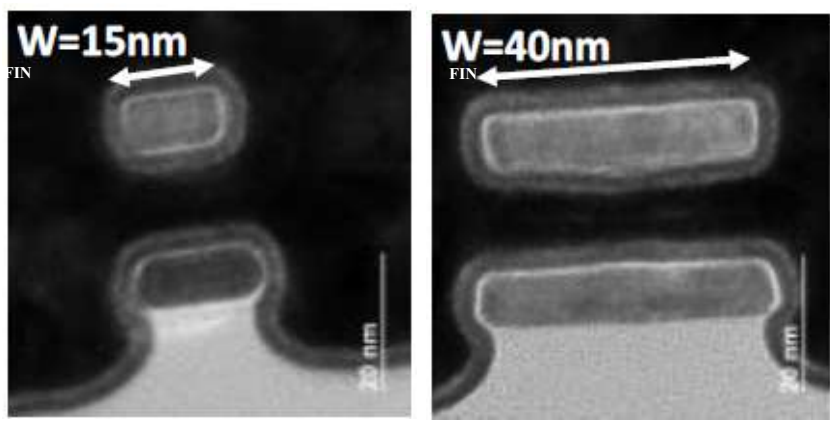

Fonte: Barraud et al., 2018

Após observar a Figura 9, se torna razoável deduzir que a largura total do nanofio verticalmente empilhado é equivalente à somatória de todos os $\mathrm{W}_{\text {FIN }}$ e $\mathrm{H}_{\text {FIN }}$ das duas camadas, conforme mostra a eq. (2): 


$$
W_{(S T A C K E D)}=W_{(T R I G A T E)}+W_{(G A A)}=4 H_{F I N}+3 W_{F I N}
$$

A altura do fin é relevante nesta análise uma vez que os valores $\mathrm{W}_{\mathrm{FIN}}$ dos nanofios empilhados não são muito maiores que os $9 \mathrm{~nm}$ de $\mathrm{H}_{\text {FIN }}$. Tomando como base a proporcionalidade entre a corrente de dreno e a relação da largura pelo comprimento de canal em dispositivos SOI $\left(I_{D S} \propto \frac{W}{L}\right)$, espera-se de fato que esse dispositivo inovador possua ótima capacidade de amplificação de corrente. A seguir, serão abordados os parâmetros elétricos relacionados à tecnologia.

\subsubsection{Parâmetros elétricos}

Antes de apresentar os resultados deste trabalho, obtidos para os nanofios empilhados, é importante discorrer a respeito das grandezas elétricas e conceitos especialmente relevantes na análise de transistores.

\subsubsection{Tensão de Limiar}

A tensão de limiar ou threshold voltage $\left(\mathrm{V}_{\mathrm{TH}}\right)$ é caracterizada como a tensão a partir da qual há formação de um canal de inversão que permita a condução expressiva de corrente elétrica entre a fonte e dreno do transistor. O campo elétrico gerado pela aplicação de tensão entre a porta e a fonte $\left(\mathrm{V}_{\mathrm{GS}}\right)$ atrai as cargas minoritárias presentes no substrato, invertendo sua polaridade na camada superficial de silício e resultando no canal por onde ocorre o trânsito dos elétrons.

Em um transistor convencional tipo nMOSFET, a tensão de limiar pode ser definida como a tensão aplicada à porta que eleva o potencial de superfície para $2 \Phi_{\mathrm{F}}$, como expresso pela eq. (3) (SZE; NG, 1981):

$$
V_{T H}=V_{F B}+2 \Phi_{F}+\frac{q N a X_{d m a x}}{C_{O X}}
$$


Sendo $\mathrm{V}_{\mathrm{FB}}$ a tensão de faixa plana, $\Phi_{\mathrm{F}}$ o potencial de Fermi, $\mathrm{X}_{\mathrm{dmax}}$ a máxima largura da região de depleção, $\mathrm{Na}$ a concentração de dopantes aceitadores do canal e $\mathrm{C}_{\mathrm{Ox}}$ a capacitância do óxido de porta por unidade de área. Além disso, sabe-se que:

$$
\begin{gathered}
V_{F B}=\Phi_{M S}-\frac{Q_{O X}}{C_{O X}} \\
\Phi_{F}=\frac{k T}{q} \ln \left(\frac{N a}{n_{i}}\right) \\
X_{\text {dmax }}=\sqrt{\frac{4 \varepsilon_{S I} \Phi_{F}}{q N a}}
\end{gathered}
$$

Onde $\Phi_{M S}$ é a diferença de função trabalho, Qox é a densidade de carga no óxido de porta, $\mathrm{k}$ é a constante de Boltzman $\left(1,38 \times 10^{-23} \mathrm{~J} / \mathrm{K}\right)$, T é a temperatura absoluta, q é a carga elementar do elétron $\left(1,6 \times 10^{-19} \mathrm{C}\right), \mathrm{n}_{\mathrm{i}}$ é a concentração intrínseca de portadores e $\varepsilon_{S I}$ é a permissividade do silício. Desta forma, é possível reescrever a eq. (3) conforme abaixo:

$$
V_{T H}=\Phi_{M S}-\frac{Q_{O X}}{C_{O X}}+2\left[\frac{k T}{q} \ln \left(\frac{N a}{n_{i}}\right)\right]+\frac{q N a \sqrt{\frac{4 \varepsilon_{S I}\left[\frac{k T}{q} \ln \left(\frac{N a}{n_{i}}\right)\right]}{q N a}}}{C_{O X}}
$$

Após notar a recorrência do termo $\mathrm{Na}$ existente na eq. (7), é possível concluir que, para um transistor MOSFET convencional, a concentração de dopantes é um fator de influência majoritária na determinação da tensão de limiar. Outro elemento que possui elevada importância na definição de $\mathrm{V}_{\mathrm{TH}}$ está relacionado à polarização do substrato, cujas equações características serão abordadas adiante, na seção 2.2.2.2.

Em um transistor SOI parcialmente depletado, a eq. (3) continua sendo válida, uma vez que a espessura do silício $\left(t_{\mathrm{si}}\right)$ permanece sendo maior do que 2 vezes a espessura máxima de depleção (assim como no transistor MOS convencional). Porém, em um SOI totalmente depletado, no qual $t_{\mathrm{si}}<\mathrm{X}_{\mathrm{dmax}}$, há uma interdependência entre os potenciais de superfície da $1^{\mathrm{a}}$ e $2^{\mathrm{a}}$ interfaces e as tensões aplicadas à porta e ao substrato, $\mathrm{V}_{\mathrm{G} 1}$ e $\mathrm{V}_{\mathrm{G} 2}$, respectivamente. $\mathrm{O}$ acoplamento dos potenciais de superfície, em função das tensões aplicadas $V_{G 1}$ e $V_{G 2}$, pode ser expresso em duas equações (COLINGE, 2004): 


$$
\begin{aligned}
& V_{G 1}=\Phi_{M S 1}-\frac{Q_{O X 1}}{C_{O X 1}}+\left(1+\frac{C_{S i}}{C_{O X 1}}\right) \Phi_{S 1}-\frac{C_{S i}}{C_{O X 1}} \Phi_{S 2}-\left(\frac{\frac{1}{2} Q_{d e p l}+Q_{i n 1}}{C_{O X 1}}\right) \\
& V_{G 2}=\Phi_{M S 2}-\frac{Q_{O X 2}}{C_{O X 2}}+\left(1+\frac{C_{S i}}{C_{O X 2}}\right) \Phi_{S 2}-\frac{C_{S i}}{C_{O X 2}} \Phi_{S 1}-\left(\frac{\frac{1}{2} Q_{d e p l}+Q_{S 2}}{C_{O X 2}}\right)
\end{aligned}
$$

Todos os índices 1 e 2 se referem à $1^{\mathrm{a}}$ e $2^{\mathrm{a}}$ interfaces (ver Figura 3), ou seja, as regiões compreendidas nas junções óxido de porta e silício (canal) e silício e óxido enterrado. Desta maneira, $\Phi_{M S 1}$ e $\Phi_{M S 2}$ são diferenças de função trabalho (metal de porta e silício) e $\Phi_{S 1}$ e $\Phi_{S 2}$ são os potenciais elétricos de superfície das regiões. Q Qx1 e Q Qx2 representam densidades de carga no óxido de porta e óxido enterrado e $\mathrm{C}_{\mathrm{ox} 1}$ e $\mathrm{C}_{\mathrm{ox} 2}$ são as capacitâncias dos óxidos por unidade de área. $\mathrm{Q}_{\text {depl }}$ é a densidade de carga total de depleção no silício, $\mathrm{Q}_{\text {in1 }}$ se refere à carga de inversão na $1^{\mathrm{a}}$ interface e Qs2 representa a carga em regime de inversão ou acumulação da $2^{\mathrm{a}}$ interface.

Finalmente, a tensão de limiar de dispositivos com tecnologia SOI (tais como os nanofios empilhados) depende da polarização em que se encontra a segunda interface, uma vez que, como visto acima, seu estado influencia a análise dos potenciais elétricos do sistema.

Para $2^{\mathrm{a}}$ interface em regime de acumulação, tem-se que o potencial relevante para o sistema se encontra apenas na $1^{\mathrm{a}}$ interface, de modo que $\Phi_{S 2} \cong 0, Q_{i n 1} \cong 0$ e $\Phi_{S 1}=2 \Phi_{F}$. Logo, a expressão da tensão de limiar se aproxima de $\mathrm{V}_{\mathrm{G} 1}$, apresentada na eq. (8):

$$
V_{T H 1, a c c 2}=\Phi_{M S 1}-\frac{Q_{O X 1}}{C_{O X 1}}+\left(1+\frac{C_{S i}}{C_{O X 1}}\right) 2 \Phi_{F}-\left(\frac{Q_{d e p l}}{2 C_{O X 1}}\right)
$$

Já para $2^{\mathrm{a}}$ interface em inversão, tem-se que $\Phi_{S 2}=2 \Phi_{F}, Q_{i n 1}=0$ e $\Phi_{S 1}=2 \Phi_{F}$, obtendo-se uma expressão de tensão de limiar igual à eq. (11). Importante constatar que nessa situação há formação de canal através da segunda interface, prejudicando o controle de chaveamento do componente, uma vez que a tensão de porta aplicada $\left(\mathrm{V}_{\mathrm{G} 1}\right)$ não controla a condução entre fonte e dreno e nem o estado ON/OFF do transistor.

$$
V_{T H 1, i n v 2}=\Phi_{M S 1}-\frac{Q_{O X 1}}{C_{O X 1}}+2 \Phi_{F}-\left(\frac{Q_{d e p l}}{2 C_{O X 1}}\right)
$$


Caso a $2^{\mathrm{a}}$ interface esteja em regime de depleção, é preciso notar que a expressão de $\mathrm{V}_{\mathrm{TH}}$ será composta pela combinação das eqs. (8) e (9). Considerando $\Phi_{S 1}=2 \Phi_{F}$ e $Q_{i n 1}=$ $Q_{S 2}=0$ e $0<\Phi_{S 2}<2 \Phi_{F}$, obtém-se:

$$
V_{T H 1, d e p l 2}=V_{T H 1, a c c 2}-\left[\frac{C_{S i} C_{O X 2}}{C_{O X 1}\left(C_{S i}+C_{O X 2}\right)}\right]\left(V_{G 2}-V_{G 2, a c c}\right)
$$

A Figura 10 esclarece a relação entre os estados de $V_{T H}$ e a tensão de substrato, que foram descritos matematicamente acima.

Figura 10 - Tensão de Limiar versus Tensão de Substrato em dispositivos SOI

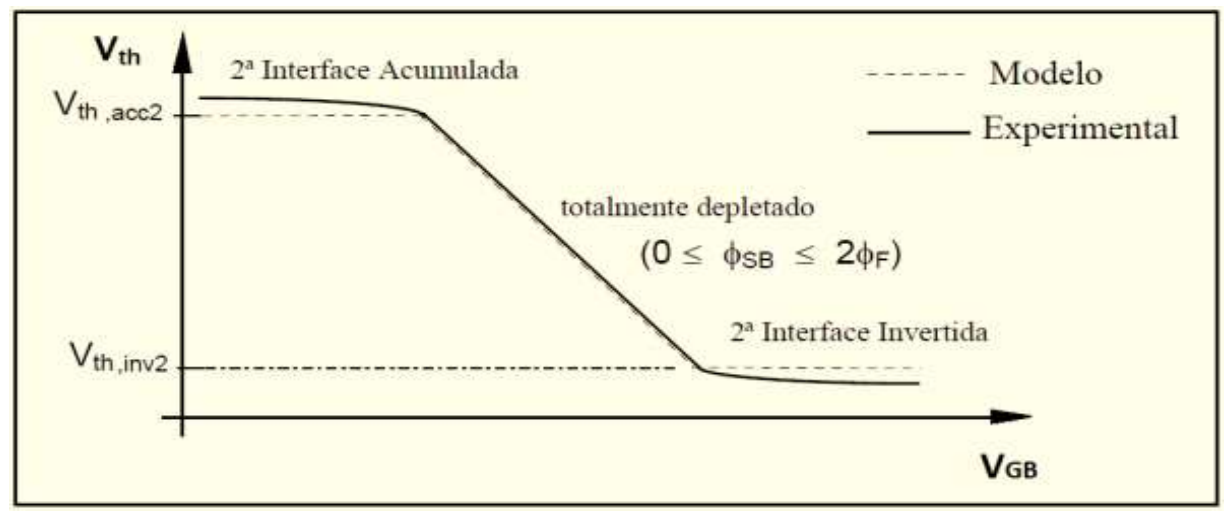

Fonte: Nemer, 2017

Na prática, existem diversos métodos para extração da tensão de limiar, a partir da curva da corrente de dreno em função da tensão aplicada a porta, como o da Segunda Derivada, Extrapolação da Região de Linear, Corrente Constante, função Y entre outros (CHENG, 2019).

Em (DUPRE, 2008) foram apresentados resultados de tensão de limiar para nanofios empilhados de 3 camadas, sendo $\mathrm{V}_{\mathrm{TH}}=0,6 \mathrm{~V}$ e $\mathrm{V}_{\mathrm{TH}}=-0,3 \mathrm{~V}$ para transistores tipo nMOS e pMOS, respectivamente. O gráfico da Figura 11 evidencia os valores de $\mathrm{V}_{\mathrm{TH}}$ obtidos para os nanofios empilhados, comparando-os a um transistor SOI totalmente depletado, ambos com L=80 nm. No gráfico, SOI_n (narrow) se refere a um transistor com largura de canal estreita $\mathrm{W}_{\text {SOI_N }}=70 \mathrm{~nm}$, enquanto o SOI_w (wide), que será apresentado adiante, representa um nanofio com canal largo de $\mathrm{W}_{\text {SOI_W }}=10 \mu \mathrm{m}$. 
Figura 11 - Curvas da corrente de dreno normalizada pela largura de canal do transistor, em função da tensão de porta

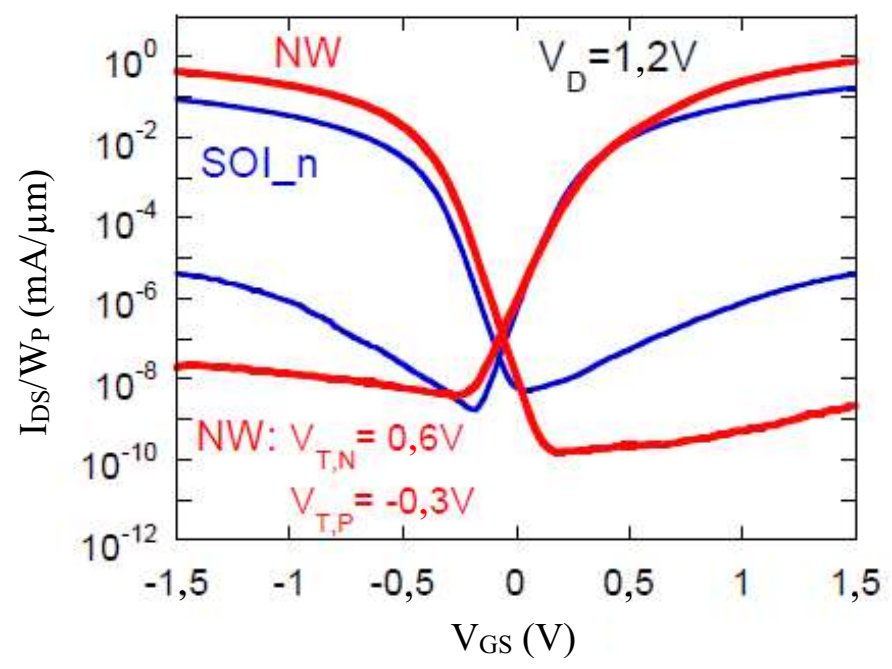

Fonte: Autor "adaptado de" Dupre, 2008

Legenda: tensão de dreno $\mathrm{V}_{\mathrm{DS}}=1,2 \mathrm{~V}$, para transistores nMOS tipo nanofio transistor empilhado com três camadas e SOI totalmente depletado, indicando as tensões de limiar

\subsubsection{Efeito de Corpo}

Tensões aplicadas ao corpo do transistor modificam sua tensão de limiar, e esta interdependência é chamada de efeito de corpo. Para entender o fenômeno, pode-se imaginar um transistor nMOSFET tradicional com tensões negativas aplicadas no corpo do componente $\left(\mathrm{V}_{\mathrm{B}}<0\right.$ e $\mathrm{V}_{\mathrm{S}}=0$, conforme ilustrado na Figura 12).

Figura 12 - nMOSFET convencional com $\mathrm{V}_{\mathrm{SB}}>0$

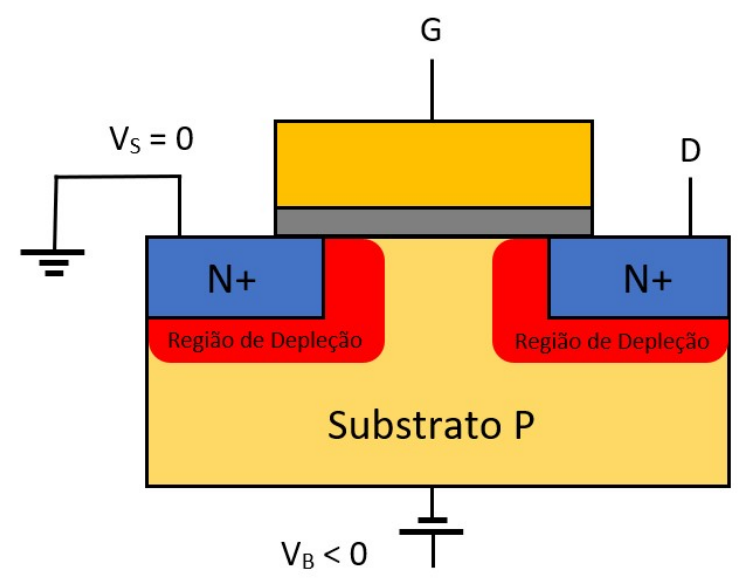

Fonte: Autor 
A tensão $\mathrm{V}_{\mathrm{SB}}>0$ provocará um alargamento na região de depleção entre a junção fonte/substrato, o que encurta a profundidade do canal e eleva a tensão de limiar.

$$
V_{T H}=V_{T H 0}+\gamma\left(\sqrt{2 \Phi_{F}+V_{S B}}-\sqrt{2 \Phi_{F}}\right)
$$

A equação acima evidencia a interrelação entre $V_{S B}$ e $V_{T H}$, de modo que $V_{T H 0}$ representa a tensão de limiar para $\mathrm{V}_{\mathrm{B}}=\mathrm{V}_{\mathrm{S}}=0 \mathrm{~V}$. O elemento multiplicador $\gamma$ é a constante de corpo, cujo valor é oriundo das características do transistor (como a permissividade e o acoplamento capacitivo):

$$
\gamma=\frac{\sqrt{2 \varepsilon_{S i} q N a}}{C_{O X}}
$$

Considerando $V_{B}$ aterrado, este efeito passa a ter dependência apenas de $V_{S}$, de forma que a eq. (13) pode ser reescrita e linearizada conforme abaixo, sendo " $n$ " o fator de corpo:

$$
\begin{gathered}
V_{T H}=V_{T H 0}+(1+\alpha) V_{S}=V_{T H 0}+n\left(V_{S}\right) \\
\alpha_{M O S}=\frac{C_{D}}{C_{O X}}
\end{gathered}
$$

Como visto anteriormente, polarizações de $2^{\mathrm{a}}$ interface em um dispositivo SOI estão intimamente ligadas à determinação de sua tensão de limiar. Adicionalmente, pode-se comentar que, semelhante ao fator de corpo relatado para os MOSFETs convencionais, no transistor SOI também existe o fator $\alpha$, que está ligado aos acoplamentos capacitivos existentes. As equações abaixo demonstram suas relações em cada uma das polarizações da $2^{\mathrm{a}}$ interface. Em acumulação:

$$
\alpha_{a c c 2}=\frac{C_{S i}}{C_{O X 1}}
$$

Já para $2^{\mathrm{a}}$ interface em depleção, tem-se: 


$$
\alpha_{d e p l 2}=\frac{C_{S i} C_{O X 2}}{C_{O X 1}\left(C_{S i}+C_{O X 2}\right)}
$$

Porém, a influência da tensão de substrato $\mathrm{V}_{\mathrm{G} 2}$ no $\mathrm{SOI}$ é menor do que os impactos causados pelo efeito de corpo nos MOS convencionais, conforme será mostrado a seguir.

\subsubsection{Inclinação de Sublimiar}

A inclinação de sublimiar (S) relaciona a variação de tensão de porta aplicada capaz de induzir variação de uma década na corrente IDS, com o transistor operando em sublimiar. Desta maneira, este parâmetro está associado à velocidade de chaveamento do transistor (ALVES, 2017), uma vez que sua sensibilidade na comutação entre ION e IOFF está diretamente ligada à magnitude de variação de $\mathrm{V}_{\mathrm{GS}}$. A equação (19) abaixo sintetiza esta característica.

$$
S=\frac{\partial V_{G S}}{\partial\left(\log \left(I_{D S}\right)\right)}
$$

$\mathrm{O}$ limite teórico à temperatura ambiente de $25^{\circ} \mathrm{C}$ para $\mathrm{S}$ é de $60 \mathrm{mV} / \mathrm{déc}$, sendo este o valor referência de comparação utilizado para mensurar a qualidade do MOSFET. Dispositivos SOI apresentam excelentes desempenhos, com valores próximos do limite teórico (WOUTERS; COLINGE; MAES, 1989) (PAVANELLO; MARTINO; FLANDRE, 2001). Ainda é possível reescrever a expressão levando-se em consideração o fator de corpo (BREWS, 1979):

$$
S=(1+\alpha) \frac{k T}{q} \ln (10)=n \frac{k T}{q} \ln (10)
$$

Conforme visto na seção 2.2.2.2, o fator de corpo está relacionado com o acoplamento capacitivo, e de acordo com 2.1.1, transistores SOI totalmente depletados possuem melhores características capacitivas em relação ao MOS convencional. Portanto, chega-se à conclusão que esta tecnologia de fato é superior no que tange à velocidade de chaveamento de componentes. Na realidade, esta afirmação é corroborada pela expressão encontrada em (COLINGE, 2004), demonstrando que $\alpha$ FD SOI $<\alpha$ MOS, já que menores valores de $\alpha$ resultam em inclinações de sublimiar mais próximas da ideal. 
Os nanofios empilhados também mostraram ótimos resultados para a corrente de dreno com o transistor operando em sublimiar, conforme pode ser visto na Figura 13, que apresenta os dados para transistores nMOS e pMOS com três camadas e $\mathrm{L}=80 \mathrm{~nm}$, medidos às tensões de dreno de $50 \mathrm{mV}$ e 1,2 V para o nMOS, e -50 mV e -1,2 V para o pMOS. Nesta figura, observa-se uma redução de quase seis ordens de grandeza da corrente elétrica quando o transistor está polarizado com tensão de porta próxima à tensão de limiar e com tensão de porta nula. Além disso, a elevação da tensão de dreno aplicada, de $50 \mathrm{mV}$ para $1,2 \mathrm{~V}$ praticamente não provocou alterações na corrente de dreno obtida com o transistor operando em sublimiar.

Figura 13 - Curva de corrente de dreno em função da polarização de porta em escala logarítmica para nanofios nMOSFET e pMOSFET empilhados $\mathrm{L}=80 \mathrm{~nm}$

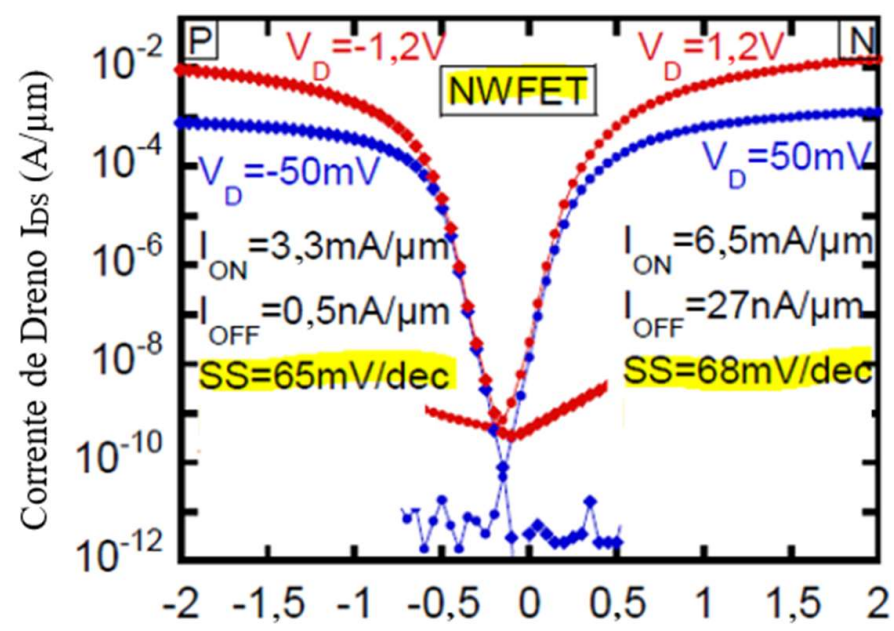

Tensão de Porta VGS (V)

Fonte: Autor "adaptado de" Dupre, 2008

\subsubsection{Mobilidade de portador}

A mobilidade $(\mu)$ se refere à propensão com que os portadores se deslocam em um material frente à ação de um campo elétrico (SZE; NG, 1981). Em um transistor, portanto, uma alta mobilidade se traduz em maior facilidade de condução de corrente elétrica no canal de silício, entre a fonte e dreno. O parâmetro que leva em consideração a mobilidade de portadores, desconsiderando-se a influência de um campo elétrico aplicado, é chamado de mobilidade de baixo campo $\left(\mu_{0}\right)$. Existem diversos fatores que impactam no trânsito dos elétrons e lacunas, resultando em efeitos de degradação de mobilidade causados pelos mecanismos de espalhamento. Alguns dos principais efeitos serão abordados a seguir. 
A vibração da estrutura cristalina do material provoca intercorrências no deslocamento dos portadores. Quanto maior a temperatura, maior a energia fornecida ao sistema e, por consequência, eleva-se a magnitude vibracional, diminuindo a mobilidade efetiva dos portadores. Tal fenômeno traduz o espalhamento por fônons, também encontrado na literatura como phonon scattering (SAH et al., 1981).

O espalhamento por impurezas ionizadas (ionized impurity scattering) se refere à dificuldade de deslocamento dos portadores em decorrência da alta concentração de dopantes no material. Em (CAUGHEY; THOMAS, 1967) foi proposto um modelo matemático que relaciona a mobilidade com a concentração de impurezas, conforme abaixo:

$$
\mu_{i i s}=\frac{\mu_{\max }-\mu_{\min }}{1+\left(\frac{N}{N_{\text {ref }}}\right)^{\alpha}}+\mu_{\text {min }}
$$

Tal dedução foi obtida empiricamente após análise de resultados experimentais publicados anteriormente (NORRIS; GIBBONS, 1967), de forma que os parâmetros $\mathrm{N}_{\text {ref, }} \alpha$, $\mu_{\max }$ e $\mu_{\min }$ foram retirados das curvas apresentadas. Quando maior a temperatura, maior o número da densidade de portadores $\mathrm{N}$, o que reduz a mobilidade efetiva.

Existem ainda dois mecanismos de relevante influência, que são: espalhamento por impurezas neutras (neutral impurity scattering) e o espalhamento portador-portador (carrierto-carrier scattering), que trazem as interações entre o deslocamento dos portadores em meio às dopantes não ionizadas e à alta densidade de portadores, respectivamente. Desta forma, conclui-se que a mobilidade de baixo campo $\left(\mu_{0}\right)$ é o parâmetro que revela a capacidade de trânsito dos portadores após consideradas as degradações dos fenômenos de espalhamento supracitados.

A mobilidade efetiva também leva em consideração a ação do campo elétrico. A inserção de tensão de porta no componente gera campo elétrico vertical $\mathrm{E}_{\mathrm{y}}$, atraindo os portadores minoritários que se mantém em contato físico com a rugosidade existente na interface entre o silício e o óxido de porta, prejudicando sua movimentação (ZHAO et al., 2009). Tal influência é mensurada através do coeficiente de degradação de mobilidade $\theta$, e a mobilidade efetiva $\mu_{\text {eff }}$ está descrita na eq. (22):

$$
\mu_{e f f}=\frac{\mu_{0}}{1+\theta\left(V_{G S}-V_{T H}\right)}
$$


Os gráficos da Figura 14 expõe os resultados das mobilidades de elétrons obtidos experimentalmente para os transistores nanofios empilhados e suas comparações com transistores SOI planares.

Figura 14 - Medições de mobilidade de elétrons em NW, SOI_w (wide) e SOI_n (narrow) versus carga de inversão

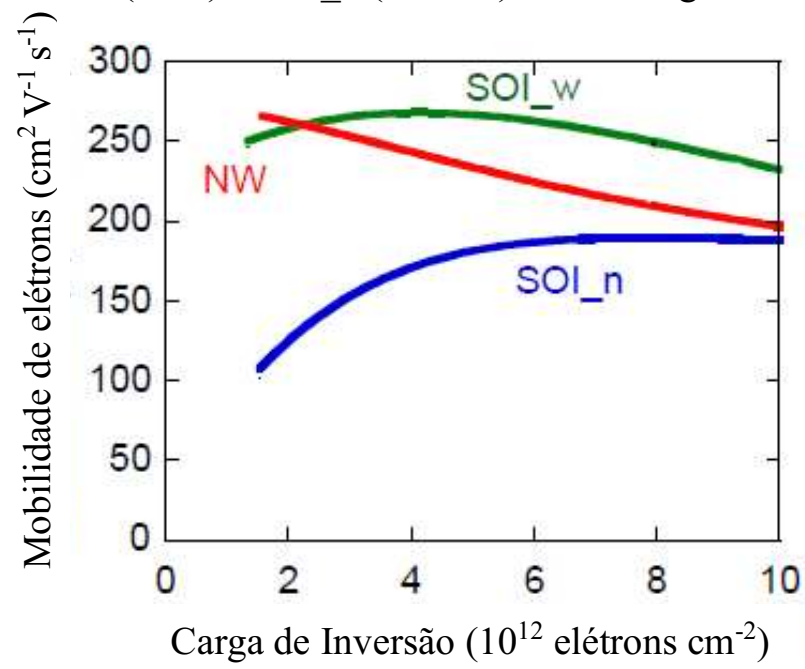

Fonte: Autor “adaptado de" Dupre, 2008

Para explicar estas curvas, é necessário primeiro recorrer ao processo de fabricação ao qual os dispositivos são submetidos. Os transistores SOI_w e SOI_n são componentes planares, enquanto os nanofios são transistores tridimensionais que possuem planos de condução paralelos e perpendiculares à lâmina de silício. Para obtê-los, o material passa por um procedimento de corrosão bastante agressivo no ato de sua confecção, o que resulta em uma deterioração do material, impactando diretamente na degradação de sua mobilidade (FECHNERAND; VOGT, 2005). Isto explica o porquê da mobilidade dos NW e SOI_W diminuírem com o aumento da carga de inversão. Outro ponto importante é que a estrutura cristalográfica das laterais do nanofio (110) não favorece a condução de elétrons, o que ajuda a entender o comportamento frente aos outros dois transistores. O NW a princípio possui maior mobilidade que o SOI_n (estreito) por possuir mais planos de condução paralelos à lâmina, mas com o aumento da carga de inversão, as suas laterais acabam por prejudicar a passagem de corrente, diminuindo consideravelmente sua mobilidade em relação ao SOI_w (largo) e aproximando-o da mobilidade do SOI_n, que é regida pela condução no topo do dispositivo, cuja estrutura cristalográfica é (100), mais propensa ao fluxo de elétrons (DUPRE, 2008). 


\subsubsection{Operação em Triodo e Saturação e Corrente de dreno IDS}

Os transistores podem operar em basicamente três regiões: corte, triodo e saturação. A região de corte se dá para tensões de porta inferiores à tensão de limiar, impedindo a formação de canal. A região de triodo ocorre a partir da formação de canal entre o dreno e a fonte caracterizado por uma tensão de porta maior do que a tensão de $\operatorname{limiar}\left(\mathrm{V}_{\mathrm{GS}}>\mathrm{V}_{\mathrm{TH}}\right)-\mathrm{e}$ a aplicação de tensão entre fonte e dreno ( $\left.V_{D S}\right)$ menor do que a diferença entre $V_{G S}$ e $V_{T H}$. Nesta configuração, o componente tem o comportamento de um resistor que é controlado a partir da tensão de porta.

Embora a região de saturação também ocorra após a formação do canal, a operação neste caso se dá em decorrência de uma tensão entre fonte e dreno maior que a diferença das tensões de porta e de limiar $\left(\mathrm{V}_{\mathrm{DS}}>\mathrm{V}_{\mathrm{GS}}-\mathrm{V}_{\mathrm{TH}}\right)$. Esse modo de funcionamento é comumente utilizado para trabalhar os transistores como amplificadores (SEDRA; SMITH, 2005), cujo desempenho está relacionado às características de distorção harmônica. Isto justifica o motivo pelo qual os dados usados na análise harmônica foram extraídos na região de saturação. A Figura 15 demonstra uma curva $I_{D S} x V_{D S}$ genérica, com o objetivo de explicitar as regiões comentadas acima.

Figura 15 - Regiões de Operação de um MOSFET genérico

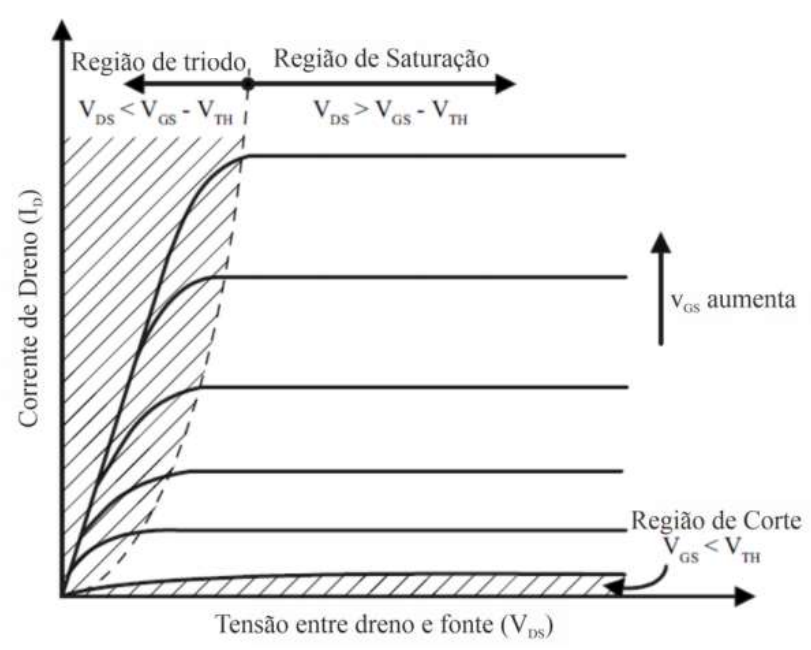

Fonte: Rosen e Samimi, 2012

Em um transistor MOSFET convencional, o modelo matemático que descreve a corrente de dreno é composto por duas equações, dependendo da região de operação. Em triodo, temos: 


$$
I_{D S(\text { triodo })}=\mu_{e f f} C_{O X} \frac{W}{L}\left[\left(V_{G S}-V_{T H}\right) V_{D S}-n \frac{V_{D S}^{2}}{2}\right]
$$

Já para a região de saturação, a corrente $\mathrm{I}_{\mathrm{DS}}$ e a tensão de saturação $\mathrm{V}_{\mathrm{DS}(\text { sat) }}$ são descritas como:

$$
\begin{gathered}
I_{D S(\text { sat })}=\frac{\mu_{e f f} C_{O X}}{2 n} \frac{W}{L}\left(V_{G S}-V_{T H}\right)^{2} \\
V_{D S(\text { sat })}=\frac{V_{G}-V_{T H}}{n}+V_{S}
\end{gathered}
$$

Realizando as devidas modificações, pode-se utilizar a eq. (24) também para os dispositivos SOI. Para tanto, basta substituir $C_{0 x}$ por $C_{0 x 1}, V_{G S}$ por $V_{G 1}$ (evidenciando a influência majoritária da $1^{\mathrm{a}}$ interface) e aplicar o coeficiente $\alpha$ adequado a cada estado de $2^{\mathrm{a}}$ interface (acumulação ou depleção), fazendo uso das eq. (17) e (18) e substituindo $n$ por $(1+\alpha)$, cuja igualdade fora mostrada na eq. (20).

O menor fator de corpo do SOI em relação ao transistor MOS tradicional não só melhora seu desempenho no que diz respeito à curva de sublimiar, mas também na capacidade de condução de corrente $I_{D S}$, uma vez que $n$ é diretamente proporcional a $\alpha$ e se encontra no denominador da eq. (24).

Um modelo matemático para a corrente de dreno de transistores multi-portas (adequado ao GAA e ao transistor de porta tripla) foi proposto em (DUARTE et al., 2013). O artigo traz equações diferentes para cada uma das regiões operacionais, mas é possível notar que, em todas as expressões, a corrente é proporcional à mobilidade de portadores $(\mu)$, além da capacitância do óxido de porta por unidade de área (Cox) de cada dispositivo. As eq. (26), (27) e (28) se referem às operações dos transistores multi-portas nas regiões de sublimiar, tríodo e saturação, respectivamente:

$$
I_{D S} \approx \frac{\mu}{L}\left(\frac{k T}{q}\right)^{2} C_{O X}\left[e^{\left(\frac{V_{G}-V_{T H}}{\frac{k T}{q}}\right)}\right]\left[\left\{1-e^{\left(\frac{-V_{D S}}{\frac{k T}{q}}\right)}\right]\right.
$$




$$
\begin{gathered}
I_{D S} \approx \frac{\mu}{L} C_{O X}\left(V_{G}-V_{T H}-V_{D S} / 2\right) V_{D S} \\
I_{D S} \approx \frac{\mu}{2 L} C_{O X}\left(V_{G}-V_{T H}\right)^{2}
\end{gathered}
$$

Na seção 2.2.1 foi apresentada a ideia de que os transistores nanofios empilhados são elementos que fornecem maiores magnitudes de corrente já que sua arquitetura por si só traz uma maior largura de canal $\mathrm{W}_{\mathrm{FIN}}$ (vide eq. (2)). A partir das informações de mobilidade efetiva mostradas na Figura 14 e o bom resultado para a curva de sublimiar evidenciado na Figura 13 (relacionado a um baixo fator de corpo), espera-se ainda mais que isto se concretize, já que estes elementos também estão presentes na equação característica de corrente do SOI e dos transistores multi-portas, além das dimensões físicas do dispositivo. De fato, a literatura comprova que esses transistores trazem um ganho de corrente em relação ao SOI planar, como pode ser visto na Figura 16.

Figura 16 - Corrente de dreno normalizada pela largura do canal em função da sobretensão de porta para transistores nanofios e SOI planares de canal largo e estreito

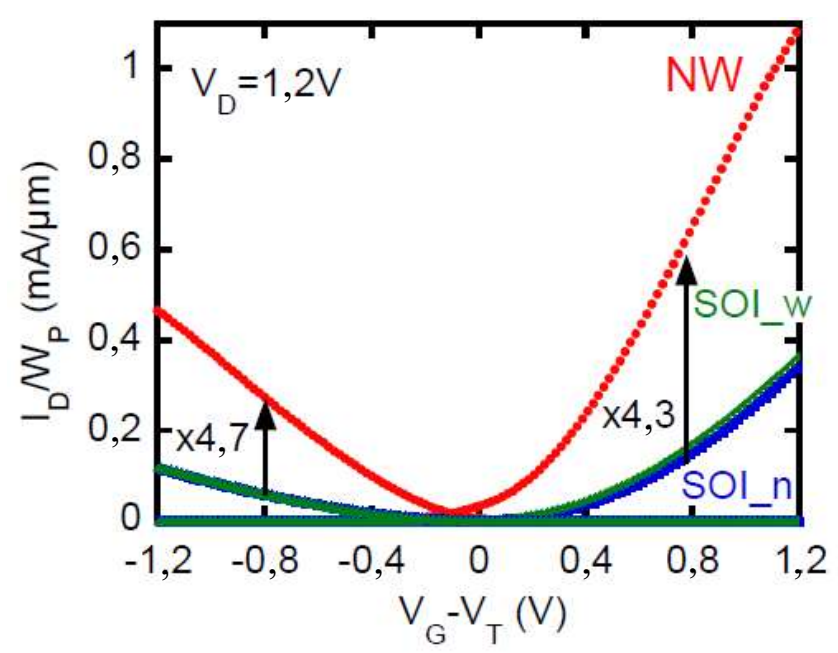

Fonte: Autor “adaptado de” Dupre, 2008

As correntes de dreno dos nanofios empilhados utilizados neste trabalho também foram extraídas e serão apresentadas no capítulo 5, uma vez que seus resultados são fundamentais para o fenômeno central de interesse da dissertação (distorção harmônica). 


\subsubsection{Transcondutância}

A transcondutância é um importante elemento de análise para aplicações analógicas de transistores, sobretudo no funcionamento de amplificadores. Ela mede a eficácia do componente no processo de conversão de tensão em corrente (mais especificamente, tensão de porta em corrente de dreno). Sua expressão matemática já foi apresentada na eq. (1), mas a partir dos novos dados e considerando a eq. (24), ela pode ser reescrita da seguinte maneira:

$$
g_{m}=\frac{\partial I_{D S(s a t)}}{\partial V_{G S}}=\frac{\mu_{e f f} C_{O X 1}}{(1+\alpha)} \frac{W}{L}\left(V_{G 1}-V_{T H}\right)
$$

Analogamente à seção anterior, deve-se empregar o fator $\alpha$ coerente para cada caso, seja o MOSFET convencional ou o SOI com a devida polarização da segunda interface. Sendo assim, e reiterando que $\alpha_{F D}$ SOI $<\alpha_{M O S}$, é factível ponderar que o SOI totalmente depletado seja superior em relação ao MOSFET, no que se refere à transcondutância. Na literatura é possível encontrar validação experimental para esta afirmação: em (FLANDRE et al., 1994) foram realizadas comparações de transcondutâncias divididas por corrente de dreno entre transistores SOI e MOS convencionais, conforme mostra a Figura 17:

Figura 17 - Comparação $\mathrm{g}_{\mathrm{m}} / \mathrm{I}_{\mathrm{DS}}$ entre SOI e MOSFET a $\mathrm{V}_{\mathrm{D}}=2,5 \mathrm{~V}$

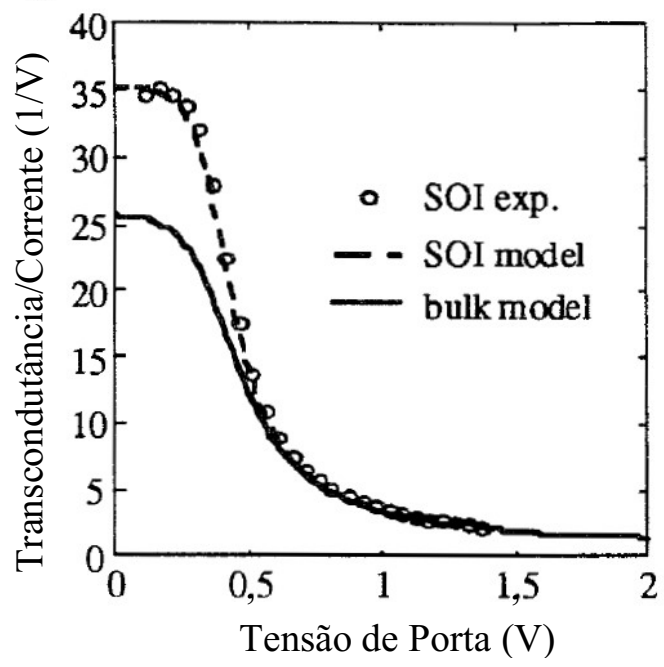

Fonte: Autor "adaptado de" Flandre et al., 1994

Nota-se que, o dispositivo SOI possui valores maiores de $\mathrm{g}_{\mathrm{m}} / \mathrm{I}$ Ds para menores valores de $\mathrm{V}_{\mathrm{GS}}$ e que ambos os componentes convergem conforme a tensão de porta aumenta. $\mathrm{O}$ gráfico 
mostra o comportamento na região de saturação (já que $\mathrm{V}_{\mathrm{DS}}=2,5 \mathrm{~V}$ ), que é a mais empregada em aplicações analógicas. A relação $\mathrm{g}_{\mathrm{m}} / \mathrm{I}_{\mathrm{DS}}$ é comumente utilizada pois ela expressa com maior precisão a eficiência do transistor, dividindo-se a amplificação pela energia necessária para se obtê-la (COLINGE, 2004). Este parâmetro $\left(\mathrm{g}_{\mathrm{m}}\right)$ é especialmente importante para as aplicações analógicas pois seu desempenho influi diretamente no ganho de tensão, conforme será descrito adiante. A Figura 18 apresenta $\mathrm{g}_{\mathrm{m}} / \mathrm{I}_{\mathrm{DS}}$ em função de $\mathrm{I}_{\mathrm{DS}}$ normalizado. A curva tem relação com o regime de inversão e o modo de operação do transistor: conforme relatado em (SILVEIRA; FLANDRE; JESPERS, 1996), $\mathrm{g}_{\mathrm{m}} / \mathrm{I}_{\mathrm{DS}}$ é máximo em regime de inversão fraca e diminui conforme passa da inversão moderada para a forte, onde o dispositivo possui maiores valores de $I_{D S} /(W / L)$ e opera em saturação.

Figura $18-\mathrm{g}_{\mathrm{m}} / \mathrm{I}_{\mathrm{DS}}$ em função de $\mathrm{I}_{\mathrm{DS}} /(\mathrm{W} / \mathrm{L})$ calculado (linha) e mensurado (símbolos) para nMOS e pMOS em um FD SOI e calculado para MOS convencionais

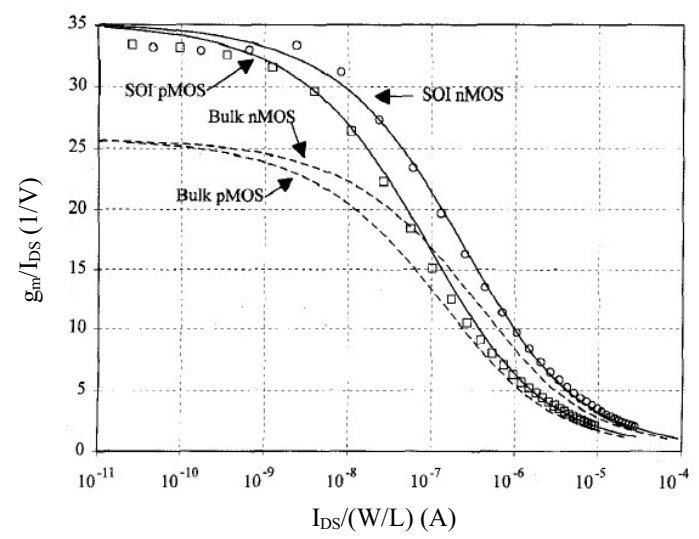

Fonte: Autor “adaptado de” Silveira, Flandre e Jespers, 1996

Em relação aos nanofios empilhados, a correlação entre temperatura e variação de $g_{m}$ pode ser conferida na Figura 19. Ela mostra com clareza que, embora menores temperaturas resultem em maiores valores de transcondutância para os dispositivos SOI, esta variação não é tão acentuada para os NW (o sentido da seta no gráfico indica diminuição de temperatura). Ainda assim, observa-se que o comportamento físico se mantém, ou seja, os valores de $\mathrm{g}_{\mathrm{m}}$ permanecem decrescendo com o aumento da temperatura, mesmo nos nanofios. No capítulo 5 serão apresentadas as transcondutâncias extraídas dos dispositivos analisados. 
Figura 19 - Transcondutância versus Tensão de porta para diferentes temperaturas em dispositivos SOI e NW

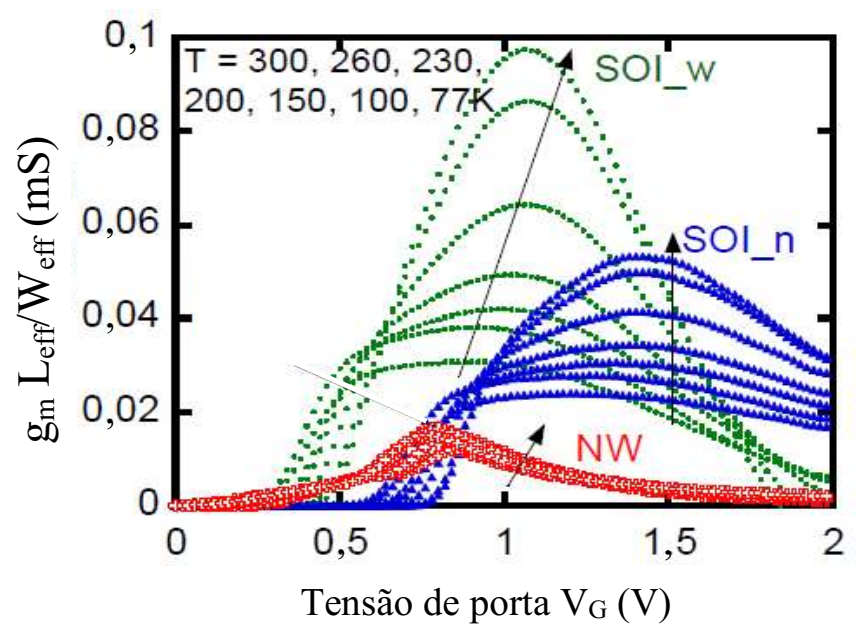

Fonte: Autor "adaptado de" Dupre, 2008

\subsubsection{Ganho de Tensão $\left(A_{V}\right)$}

Um dos principais elementos de um transistor operando como amplificador é o seu ganho de tensão em malha aberta $\left(\mathrm{A}_{\mathrm{V}}\right)$. Ele define a capacidade de amplificação de um sinal de entrada em um circuito que não possui realimentação. Este fator é proporcional à relação da transcondutância pela condutância de saída e igual à transcondutância dividida pela tensão de dreno e multiplicada pela tensão Early $\left(\mathrm{V}_{\mathrm{EA}}\right)$, eq. (30). A transcondutância já foi definida anteriormente e a condutância de saída está indicada abaixo (eq. (31)), sendo a relação da variação de corrente de dreno obtida com a variação de $V_{D S}$.

$$
\begin{gathered}
\left|A_{V}\right|=\frac{g_{m}}{g_{D}}=\frac{g_{m}}{I_{D S}} V_{E A} \\
g_{D}=\frac{\partial I_{D S}}{\partial V_{D S}}
\end{gathered}
$$

Os benefícios de se utilizar tecnologia SOI para este parâmetro também se aplicam. A Figura 20 faz uma comparação de dispositivos MOS e SOI operando como amplificadores, indicando melhores desempenhos para o SOI: maior ganho por área e menor dissipação de energia em stand-by quando comparado com o MOS convencional. 
Figura 20 - Amplificador de Miller com $\mathrm{C}=10 \mathrm{pF}$ e $\mathrm{f}_{\mathrm{T}}=10 \mathrm{MHz}$ para um transistor MOS (-) e um SOI (- - )

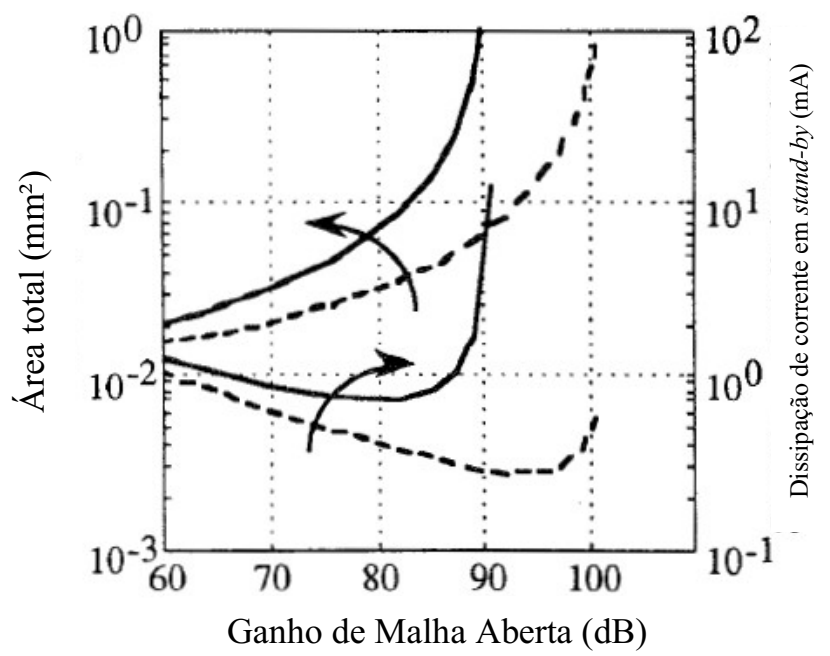

Fonte: Autor "adaptado de" Flandre et al., 1994

Os ganhos de tensão dos nanofios verticalmente empilhados usados nessa dissertação foram extraídos e seus dados serão empregados nas discussões dos resultados, no capítulo 5 . Embora tenha-se dado um passo importante em direção ao entendimento do comportamento elétrico dos transistores, conceituando seus principais parâmetros através de suas equações características e dependências, além de consolidar comparações, relações, semelhanças e dados da literatura, ainda não se discutiu a respeito do fenômeno físico de interesse específico da dissertação, que é a distorção harmônica, especialmente importante entre os circuitos analógicos e mistos. Portanto, o próximo capítulo se dedicará especialmente a tratar deste parâmetro, abordando o efeito sob o prisma matemático e de suas influências em aplicações eletrônicas diversas. Importante ressaltar que a distorção harmônica dos nanofios verticalmente empilhados até o momento não foi explorada, indicando a relevância e motivação da pesquisa. 


\section{DISTORÇÃO HARMÔNICA}

As seções abaixo se dedicam a apresentar uma boa compreensão da não-linearidade harmônica, munindo-se de conceitos matemáticos e análises de sinais. Em uma segunda etapa, serão abordadas as influências da distorção no universo da eletrônica, desde circuitos simples até impactos no setor industrial, a fim de demonstrar a importância do seu estudo.

\subsection{CONCEITOS E DEFINIÇÕES}

A distorção harmônica está relacionada à qualidade do sinal elétrico resultante após a atuação de um componente, circuito ou sistema eletrônico. Tendo como base um sinal senoidal puro com frequência fundamental igual a " $\omega \mathrm{t}$ " sendo inserido na entrada de um circuito amplificador, tem-se que a distorção harmônica total (THD) é a somatória de todas as contribuições harmônicas indesejadas geradas pela eletrônica no processo de amplificação e inseridas no sinal original. Isto ocorre em decorrência da natureza não-linear dos dispositivos empregados no circuito, como é o caso dos transistores. Os gráficos da Figura 21 demonstram o exemplo, indicando a fundamental e seus harmônicos em um circuito hipotético. A eq. (32) traduz matematicamente que o sinal resultante é uma somatória de suas componentes harmônicas.

Figura 21 - Frequência fundamental e seus harmônicos

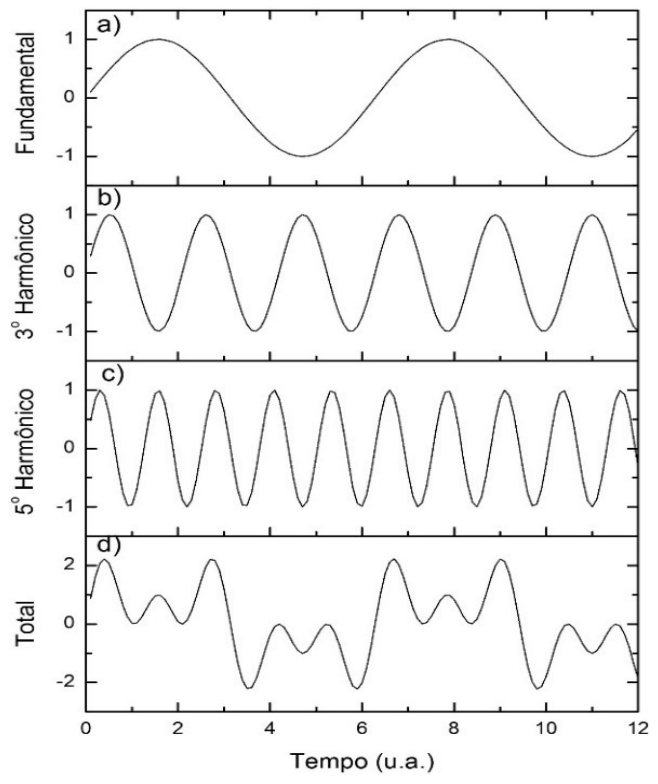

Fonte: Azevedo, Romão e Menegatti, 2019 


$$
V_{\text {Total }}(t)=V_{F} \operatorname{sen}\left(\omega t+\varphi_{F}\right)+V_{3} \operatorname{sen}\left(3 \omega t+\varphi_{3}\right)+V_{5} \operatorname{sen}\left(5 \omega t+\varphi_{5}\right)
$$

Os harmônicos citados no exemplo anterior se referem às componentes senoidais cujas frequências são múltiplas da fundamental. No exemplo, todos os harmônicos são ímpares, mas não é incomum para o circuito gerar componentes pares. A magnitude da distorção causada pelo primeiro harmônico superior (segundo harmônico) é representada pela simbologia "HD2", enquanto a não-linearidade proveniente do terceiro harmônico é simbolizada por "HD3", e assim por diante. A THD engloba todas as frequências pares e ímpares geradas e somadas ao sinal de origem, porém as primeiras harmônicas geralmente possuem maior relevância, já que estas normalmente possuem amplitudes mais próximas da frequência fundamental do que os harmônicos de ordens mais altas, cujas amplitudes muitas vezes são desprezíveis.

Uma estratégia muito utilizada na medição e visualização das distorções harmônicas é auferir o sinal de interesse no domínio da frequência. Isto é possível a partir de instrumentos que possuam a função FFT (Fast Fourier Transformer), que separa o sinal em seus respectivos harmônicos. Maiores detalhes da transformada e série de Fourier serão explicados no capítulo 4. A Figura 22 traz uma foto da tela de um osciloscópio medindo o sinal senoidal da rede elétrica, sobrepondo a análise no domínio do tempo (em amarelo) e a visualização no domínio da frequência (em azul).

Figura 22 - Senóide mensurada no domínio do tempo e da frequência

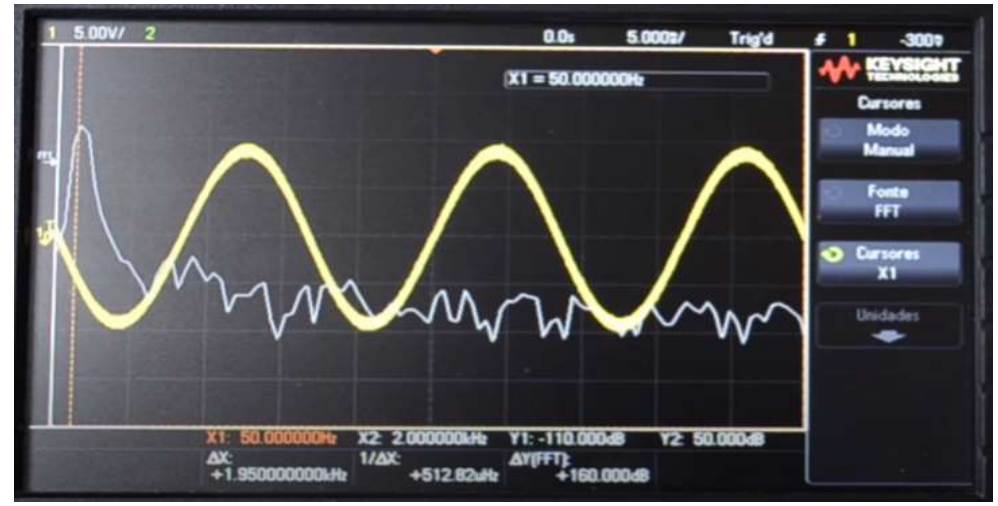

Fonte: Benfica, 2017

Nota-se que para um sinal pouco distorcido, a amplitude da frequência fundamental (primeiro pico azul) se mostra muito mais relevante do que seus respectivos harmônicos superiores. Importante ressaltar que esta representação está em escala logarítmica, segregando ainda mais as magnitudes dos níveis de tensão para as diferentes frequências. 
Vale ainda comentar sobre outro ponto interessante: a relação direta entre a simetria do sinal e os tipos de harmônicos. A Figura 23 mostra uma senóide fundamental em sobreposição ao seu quarto e quinto harmônico, respectivamente nos itens a) e b). As somatórias das frequências podem ser vistas nos itens c) e d).

Figura 23 - a) Fundamental e $4^{\circ}$ Harmônico. b) Fundamental e $5^{\circ}$ Harmônico.

c) Somatória dos sinais de a). d) Somatórias dos sinais de b)
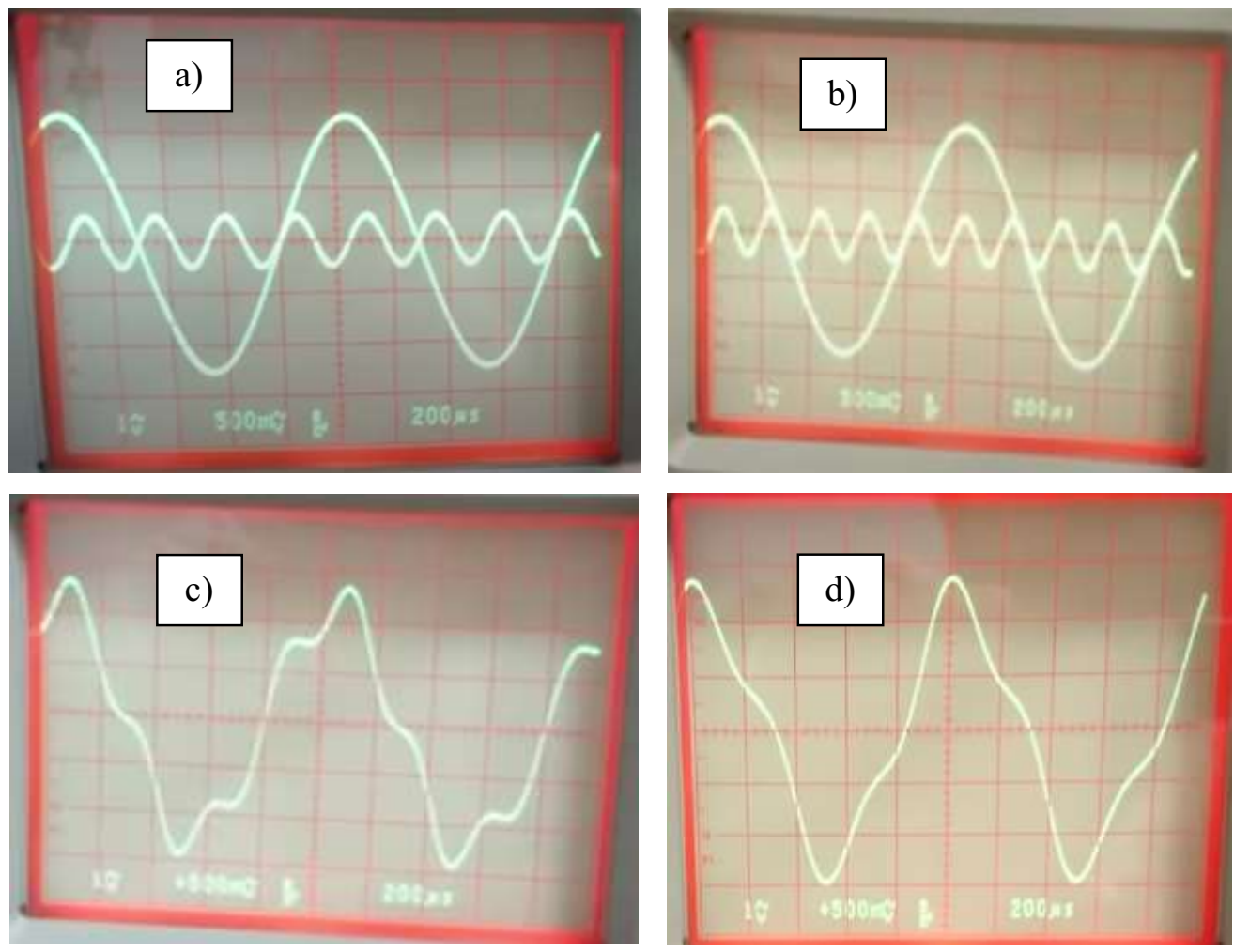

Fonte: Gusberti, 2018

É possível perceber que a somatória dos harmônicos pares - item c) - gera um sinal assimétrico no semiciclo positivo em relação ao negativo, enquanto a composição feita com harmônicos ímpares - item d) - gera uma onda simétrica em ambos os semiciclos. Isto explica o porquê das ondas quadradas, que são perfeitamente simétricas, serem frutos da somatória de infinitos harmônicos ímpares.

Em relação aos transistores, sabe-se que as distorções harmônicas de segunda e terceira ordem podem ser calculadas conforme as equações (33) e (34), respectivamente (GROENEWOLD; LUBBERS, 1994): 


$$
\begin{gathered}
H D 2=\frac{V_{A}}{4} \times \frac{\frac{\partial g_{m}}{\partial V_{G T}}}{g_{m}} \\
H D 3=\frac{V_{A}{ }^{2}}{24} \times \frac{\frac{\partial^{2} g_{m}}{\partial V_{G T}}}{g_{m}}
\end{gathered}
$$

Ambas as fórmulas demonstram que a distorção harmônica é diretamente proporcional à variação da transcondutância pela sobretensão de condução $\left(V_{\mathrm{GT}}=\mathrm{V}_{\mathrm{GS}}-\mathrm{V}_{\mathrm{TH}}\right)$, e essa questão será melhor discutida e abordada no capítulo 5, onde serão evidenciados os resultados obtidos para os nanofios verticalmente empilhados, objetivo central deste trabalho.

Apesar do embasamento teórico acima, ainda não foi demonstrado qual o impacto prático da distorção harmônica nos circuitos e sistemas elétricos. A próxima etapa elencará algumas das consequências causadas por este fenômeno, reforçando a importância do seu estudo.

\subsection{ORIGENS E CONSEQUÊNCIAS DA DISTORÇÃO HARMÔNICA}

Embora o trabalho se dedique a estudar a distorção harmônica de um único transistor, os impactos deste fenômeno em sistemas mais complexos são atualmente conhecidos. O estudo da distorção harmônica do estado da arte se torna mais relevante à medida que se nota que este é o primeiro elemento a contribuir com a não linearidade total do sistema, e sua distorção será amplificada em diversos níveis ao longo da aplicação final. Portanto, faz-se necessário discutir as consequências práticas e conhecidas da não linearidade, uma vez que o nanofio empilhado poderá, no futuro, compor parte destes circuitos.

Sinais elétricos repletos de distorção harmônica podem trazer uma série de implicações. Sua geração e seus impactos são bem pronunciados na indústria, já que muitas vezes estes setores possuem equipamentos de alta potência "sujando" a rede elétrica e utilizando essa mesma rede em suas aplicações. Abaixo serão citadas algumas das causas e consequências relacionadas a esta perturbação de sinal. 


\subsubsection{Origens da distorção harmônica}

Todo tipo de carga não linear ligada à rede se torna um elemento causador de distorção harmônica (NOVELIN, 2017). Antigamente, os equipamentos elétricos utilizados em residências eram predominantemente resistivos (chuveiro elétrico, lâmpada incandescente, ferro elétrico etc.), com uma natureza de consumo elétrico linear. Com a evolução tecnológica dos produtos, esse panorama se tornou totalmente diferente: hoje, a maioria dos eletrodomésticos possuem componentes geradores de distorção, como fontes chaveadas, lâmpadas LED e fluorescentes compactas com baixo fator de potência. Tendo em vista que a quantidade é pequena e as potências são baixas no ambiente doméstico, suas contribuições individuais normalmente não são de extrema relevância para a rede residencial, porém a situação muda no âmbito industrial.

Fábricas que utilizam motores elétricos possuem o desafio de acioná-los sem promover picos de corrente agressivos em sua rede. Partidas eletromecânicas eram empregadas no passado (partida direta, estrela-triângulo, chave compensadora etc.), e algumas das técnicas de fato atenuavam em partes os picos de corrente de partida. Porém, foi somente após o advento dos soft-starters e conversores de frequência (popularmente chamados de "inversores de frequência”) que se obteve uma minimização realmente considerável deste efeito, além dos enormes ganhos de controle, desempenho e proteção dos motores. Em detrimento de tantos benefícios, estes equipamentos passaram a integrar uma infinidade de plantas industriais, além de produtos acabados cujo controle de motores elétricos se faz necessário. A Figura 24 demonstra o esquema elétrico de potência de um inversor de frequência genérico.

Figura 24 - Circuito de um Inversor de Frequência e o comportamento dos sinais em suas diferentes etapas

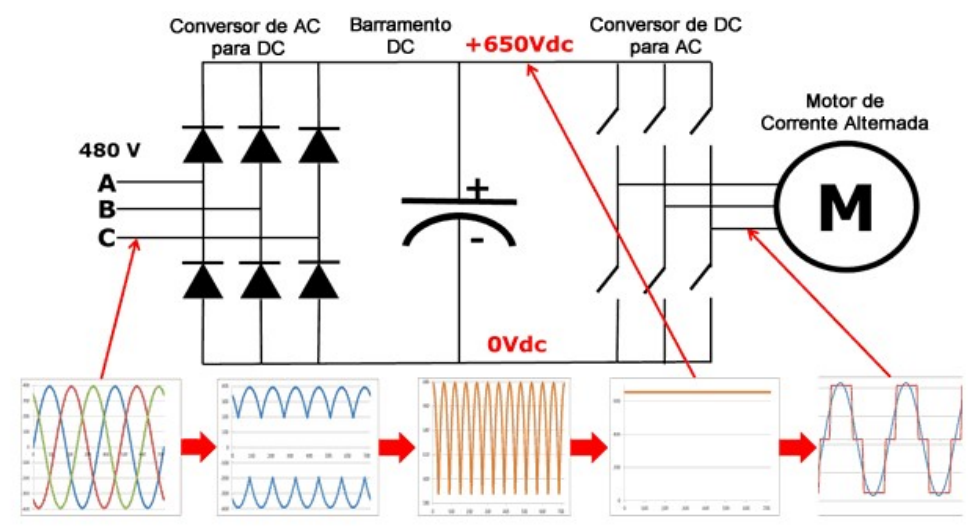

Fonte: Silveira, 2019 
O grande problema é que se por um lado os inversores de frequência representam um grande avanço no acionamento de motores, por outro são um dos grandes geradores de distorção harmônica na rede industrial em decorrência de suas etapas de conversoras AC / DC e DC / AC (ARRIGALA; WATSON, 2003). Alguns problemas relacionados a isto serão elencados a seguir.

\subsubsection{Desdobramentos da distorção harmônica}

A importância do estudo da distorção harmônica em uma nova tecnologia de transistor está diretamente associada ao conhecimento de suas consequências. Redes elétricas com elevadas taxas de distorção harmônica promovem o "skin effect" (ou efeito pelicular), onde os elétrons da corrente transitam na faixa mais externa do condutor para frequências mais elevadas, provocando o sobreaquecimento da fiação, envelhecimento precoce do isolamento, maior queda de tensão na linha e consumo extra de energia (MERRIKHI; MOGHANI; FALLAH, 2006). A Figura 25 ilustra este fenômeno. O sobreaquecimento do condutor também prejudica o enrolamento das bobinas dos motores elétricos e transformadores, cujas vidas úteis podem ser reduzidas e seu desempenho, prejudicado. Além disso, o efeito pode ocasionar a queima de fusíveis e mal funcionamento de relés e disjuntores (ORTMEYER; CHAKRAVARTHI, 1985).

Figura 25 - Representação do efeito pelicular e sua relação com a frequência do sinal

\section{EFEITO PELICULAR (ALTA FREQUÊNCIA)}

Profundidade de penetração de corrente (em azul)
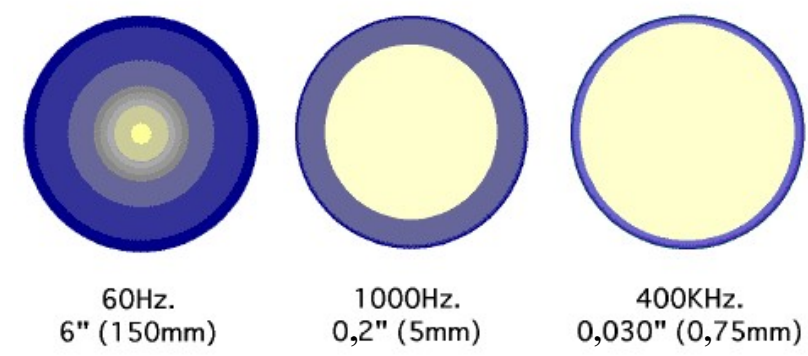

Fonte: Autor “adaptado de" Espinoza, 2012

Outro ponto importante está associado aos elementos com cargas capacitivas e indutivas. Tendo em vista que tanto a reatância capacitiva quanto indutiva depende da frequência (eq. (35) e (36), abaixo), fica simples perceber que a existência de diferentes harmônicos - e portanto frequências - altera o comportamento destas grandezas. De fato, os 
bancos de capacitores empregados na indústria com função de correção do fator de potência são impactados pelas distorções harmônicas causadas por outros dispositivos, podendo até danificá-los (SANTOS, 2010).

$$
\begin{aligned}
& X_{C}=\frac{1}{2 \pi f C} \\
& X_{L}=2 \pi f L
\end{aligned}
$$

A distorção harmônica também tem impacto nos circuitos de áudio. A THD é um elemento comumente utilizado para mensurar a qualidade dos circuitos amplificadores e reprodutores de som. Novas tecnologias continuam surgindo neste setor, sempre almejando alcançar dispositivos com maior capacidade de reprodução "high fidelity", objetivo este que está diretamente relacionado a circuitos amplificadores com baixa distorção harmônica (MEHTA; HUIJSING; STOJANOVIC, 2019).

Uma vez discutidos alguns dos efeitos da distorção harmônica, é possível compreender que os resultados neste trabalho poderão servir de base na avaliação do emprego dos nanofios empilhados em novos circuitos elétricos, uma vez que os dados obtidos permitem aproximar ou afastar a viabilidade de seu uso em função da aplicação e de cada necessidade específica.

Até o momento, apresentou-se o transistor e o fenômeno de interesse para o trabalho. Resta agora compreender a metodologia empregada no tratamento dos sinais experimentalmente obtidos, que permitiram extrair esta característica analógica. 


\section{METODOLOGIA}

Neste capítulo serão abordadas duas das principais técnicas utilizadas na extração a distorção harmônica. Após discutir os pontos envolvidos, ficará claro o motivo pelo qual o Método da Função Integral fora escolhido.

\subsection{SÉRIE E TRANSFORMADA DE FOURIER}

A seção 3.1 introduziu o método de Fourier, mostrando que aparelhos de análise de sinais transformam o sinal de interesse em componentes no domínio da frequência. Esta técnica é denominada transformada rápida de Fourier $(F F T)$ e é amplamente utilizada na determinação da não-linearidade de circuitos, sobretudo para sinais com componentes não-periódicos. Em se tratando de sinais periódicos, é possível aplicar a série de Fourier, cuja fórmula matemática (eq. (37)) demonstra que todo sinal periódico é uma somatória de senóides e cossenóides, acrescidas de um sinal contínuo.

$$
f(x)=\frac{a_{0}}{2}+\sum_{n=1}^{\infty}\left[a_{n} \cos (n x)+b_{n} \operatorname{sen}(n x)\right] ; \text { onde } x=\omega t
$$

A contribuição da incógnita $\mathrm{a}_{0}$ diz respeito ao sinal DC comentado acima, ele pode ser definido pela eq. (38):

$$
a_{0}=\frac{1}{\pi} \int_{0}^{2 \pi} f(x) d x
$$

Já as amplitudes referentes a um harmônico " $n$ " qualquer podem ser obtidos através das eq. (39) e (40):

$$
\begin{aligned}
& a_{n}=\frac{1}{\pi} \int_{0}^{2 \pi} f(x) \cos (n x) d x \\
& b_{n}=\frac{1}{\pi} \int_{0}^{2 \pi} f(x) \operatorname{sen}(n x) d x
\end{aligned}
$$


Os termos trigonométricos são úteis para demonstrar as formas de onda que contribuem na formação do sinal de interesse, porém para efeito de análise da não-linearidade, os coeficientes de amplitude são os fatores determinantes. Desta maneira, a magnitude da distorção harmônica de ordem "n" referenciada em relação à frequência fundamental pode ser calculada conforme eq. (41) $\left(\mathrm{HD}_{\mathrm{n}}\right)$. Como consequência, a distorção harmônica total (THD) representa a somatória das contribuições para todos os harmônicos gerados pela não-linearidade do sistema, como pode ser visto na eq. (42).

$$
\begin{gathered}
H D_{n}=\sqrt{\frac{a_{n}{ }^{2}+b_{n}{ }^{2}}{a_{1}{ }^{2}+b_{1}{ }^{2}}} \\
T H D=\sqrt{\frac{\sum_{n=2}^{\infty}\left\{a_{n}{ }^{2}+b_{n}{ }^{2}\right\}}{a_{1}{ }^{2}+b_{1}{ }^{2}}}
\end{gathered}
$$

Como visto na seção 3.1, na prática o algoritmo dos analisadores de espectro cria uma imagem das componentes de frequência na tela, permitindo ao pesquisador visualizar a distorção harmônica através da comparação entre o sinal de entrada e de saída. Para um transistor, isso significa que é preciso mensurar os sinais de corrente do dispositivo. O problema é que no caso de semicondutores nanométricos de baixa potência, como os nanofios empilhados, os sinais de corrente são da ordem de $\mu \mathrm{A}$, e portanto, os ruídos inerentes ao processo de medição tem enorme impacto no sinal obtido, não permitindo dissociar e definir a real não-linearidade proveniente especificamente do transistor. Neste sentido, o método da função integral se torna extremamente útil.

\subsection{MÉTODO DA FUNÇÃO INTEGRAL (IFM)}

O IFM (integral function method) é uma ferramenta que possibilita obter as distorções harmônicas de dispositivos e circuitos através de suas características DC. Seu método foi apresentado em 2004 (CERDEIRA et al., 2004) e foi escolhido como a técnica mais apropriada no processamento de sinais deste trabalho.

A utilização dos sinais contínuos elimina os erros gerados pela medição AC comentados anteriormente, e ainda assim, apresentam resultados muito satisfatórios para os harmônicos de primeira, segunda e terceira ordem (que são os mais relevantes), além da distorção harmônica 
total. A técnica consiste em algumas etapas: primeiro, é utilizado um artifício matemático que representa a aplicação de uma senóide de amplitude "V $\mathrm{V}_{\mathrm{A}}$ " e nível DC " $\mathrm{X}_{0}$ " na porta do transistor (entrada). Levando-se em consideração a curva característica de $\mathrm{I}_{\mathrm{DS}} \mathrm{x} \mathrm{V}_{\mathrm{GS}}$ já conhecida e normalizada, a saída do sistema pode ser obtida em função dos parâmetros de entrada (eq. (43)).

$$
y(x)=\frac{Y\left[X_{0}+V_{A}(2 x-1)\right]-Y\left(X_{0}-V_{A}\right)}{Y\left(X_{0}+V_{A}\right)-Y\left(X_{0}-V_{A}\right)}
$$

Segundo (CERDEIRA et al., 2004), a área compreendida entre a reta normalizada e a curva original, denominada “D” e descrita pela eq. (44), pode ser empregada no cálculo do THD (eq. (45)).

Figura 26 - Processo de normalização da curva DC para o IFM

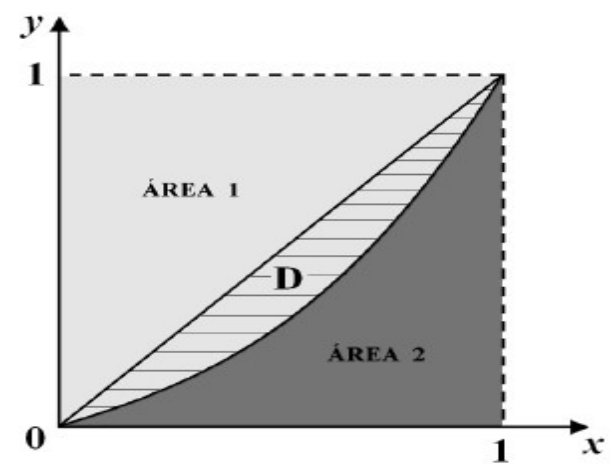

Fonte: Doria, 2010

$$
\begin{aligned}
D & =\left|2 \int_{0}^{1} y(x) d x-1\right| \\
T H D & =\sqrt{(1,06 D)^{2}-\frac{\left|C_{0}\right|^{2}}{\left|C_{1}\right|^{2}}}
\end{aligned}
$$

O IFM foi implementado na ferramenta matemática MathCAD e utilizado na extração das distorções harmônicas dos nanofios verticalmente empilhados, cujas curvas IDS $x V_{G S}$ foram extraídas experimentalmente. Na prática, após aplicação da senóide em toda a extensão da curva característica de corrente de dreno, o software realiza os cálculos descritos acima e devolve uma matriz de resultados de não linearidade. A Figura 27 demonstra o processo, onde são 
contabilizadas as contribuições da não linearidade em toda a excursão do eixo X para a curva $\mathrm{I}_{\mathrm{DS}} \mathrm{x} \mathrm{V}_{\mathrm{GS}}$ acrescida da senóide com amplitude $\mathrm{V}_{\mathrm{A}}$. A Figura 28, por sua vez, indica um resultado genérico gerado após a utilização do IFM implementado em MathCAD, onde $V_{0}$ representa $V_{G}$. O Anexo A traz uma representação do processo do IFM em forma de fluxograma, para melhor compreensão.

Figura 27 - Aplicação da senóide na curva IDS x $V_{G S}$ de um transistor

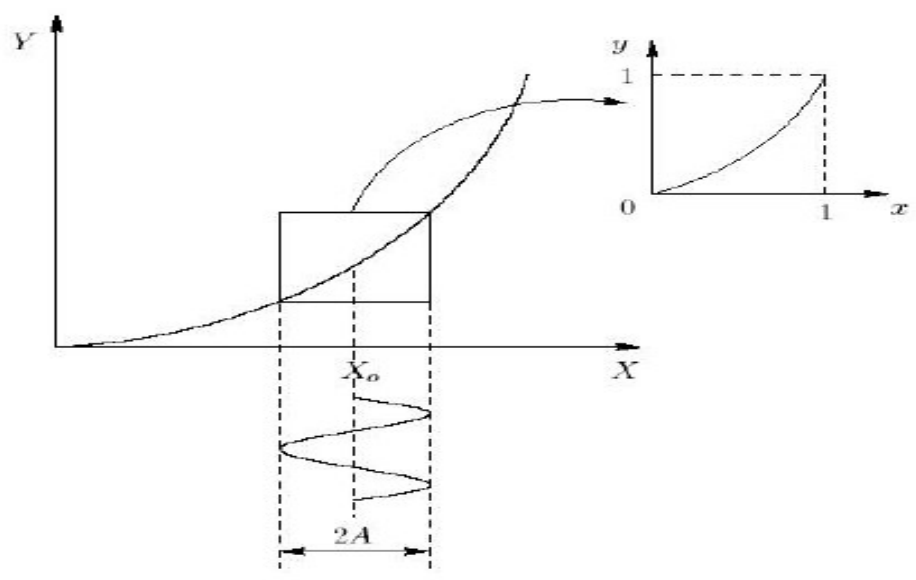

Fonte: Doria, 2010

Figura 28 - Resposta típica de THD em função da tensão de polarização, obtida no MathCAD para um nanofio empilhado

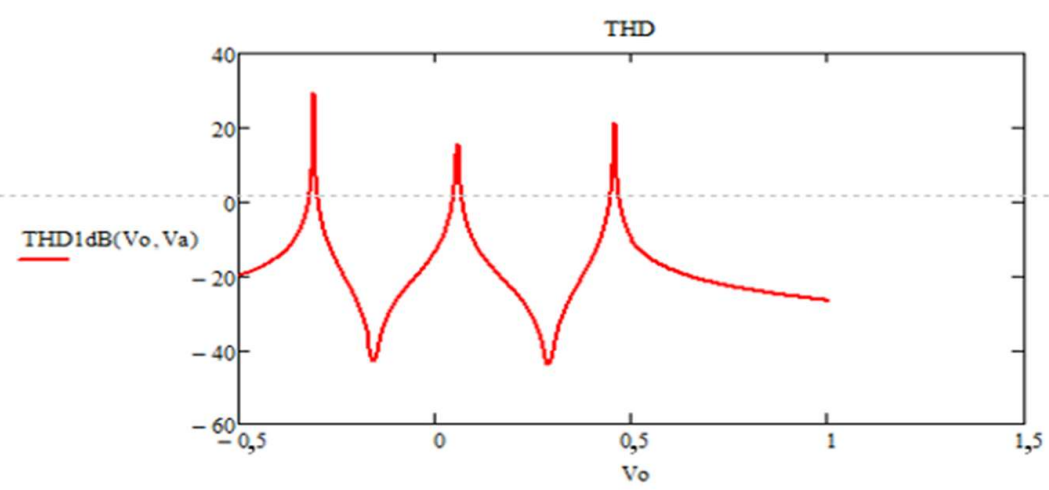

Fonte: Autor

Em posse das curvas experimentais e do software com a metodologia mais apropriada, foram realizados diversos processamentos de sinais com o objetivo de explorar e elucidar o comportamento do novo transistor frente à sua não-linearidade. Tais resultados, discussões, considerações e conclusões serão abordados a seguir. 


\section{RESULTADOS EXPERIMENTAIS}

Os primeiros experimentos realizados no laboratório de nanoeletrônica do Centro Universitário FEI permitiram extrair algumas características elétricas dos nanofios empilhados. As medidas foram realizadas em temperatura ambiente e utilizando um equipamento analisador de parâmetros de semicondutores (Keysight B1500A). As amostras disponíveis possuíam transistores nanofios empilhados tipo $\mathrm{n}$ com diversas dimensões: de um lado, $\mathrm{W}_{\text {FIN }}$ fixo com $10 \mathrm{~nm}$ e comprimento de canal (L) variável de $100 \mathrm{~nm}, 200 \mathrm{~nm}$ e $400 \mathrm{~nm}$, e de outro lado, L fixo com $100 \mathrm{~nm}$ e largura do fin variável de $10 \mathrm{~nm}, 15 \mathrm{~nm}, 20 \mathrm{~nm}$ e $40 \mathrm{~nm}$. Nas medições, foram aplicadas tensões entre o dreno e a fonte de $25 \mathrm{mV}$ e $750 \mathrm{mV}\left(\mathrm{V}_{\mathrm{DS}}=25 \mathrm{mV}\right.$ e $\mathrm{V}_{\mathrm{DS}}=750$ $\mathrm{mV}$ ), dependendo do parâmetro que se almejava obter, conforme será mostrado adiante.

Ambas as camadas possuem fin com altura de $9 \mathrm{~nm}\left(\mathrm{H}_{\mathrm{FIN}}\right)$, e a porta que as envolve é composta por uma camada de óxido de silício $\left(\mathrm{SiO}_{2}\right)$, com espessura de $1 \mathrm{~nm}$, seguida por uma camada de óxido de háfnio $\left(\mathrm{HfO}_{2}\right)$ com $2 \mathrm{~nm}$ de espessura, seguida por uma camada de $5 \mathrm{~nm}$ de espessura de nitreto de titânio (TiN) e, finalmente, por uma camada espessa de tungstênio (W). Seu óxido enterrado está localizado abaixo do primeiro nível (transistor Trigate) e possui $145 \mathrm{~nm}$ de espessura. A Figura 29 traz imagens dos transistores nanofios empilhados com diferentes $\mathrm{W}_{\mathrm{FIN}}$, obtidos utilizando processo de fabricação similar ao dos dispositivos medidos nessa dissertação.

Figura 29 - Imagens TEM de Nanofios Empilhados com $\mathrm{W}_{\text {FIN }}=10 \mathrm{~nm}$ e $\mathrm{W}_{\text {FIN }}=5 \mathrm{~nm}$

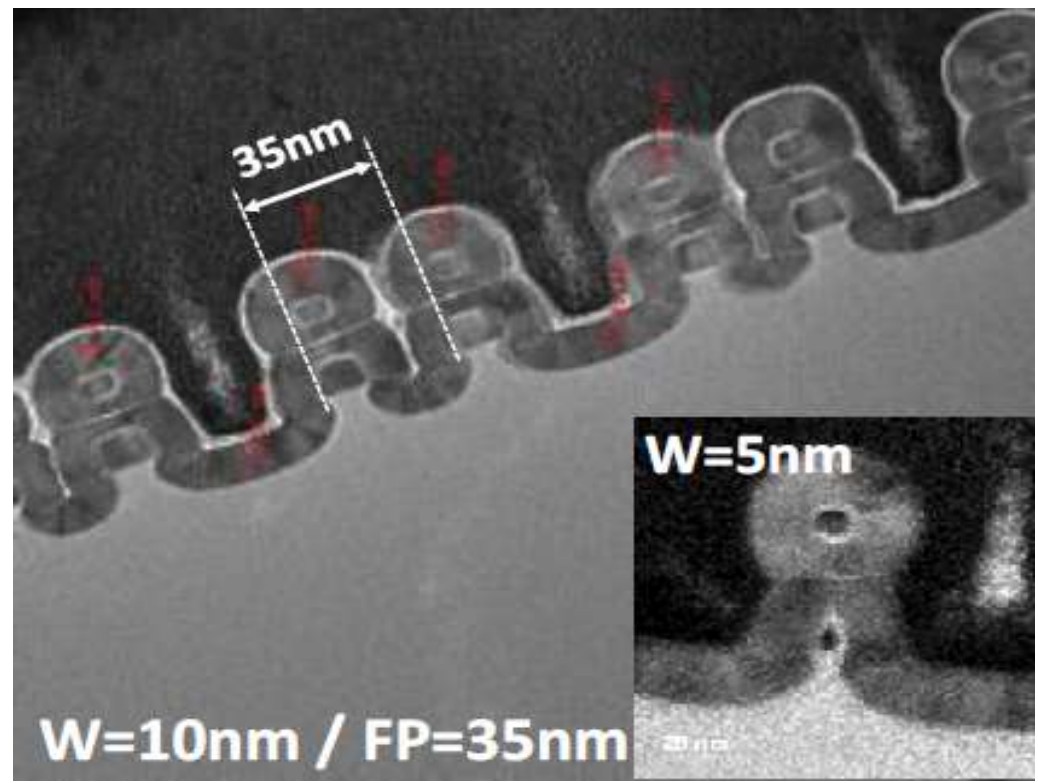

Fonte: Barraud et al., 2018 


\subsection{EXTRAÇÃO DE PARÂMETROS GERAIS}

A seguir estão descritos os resultados de diversos parâmetros elétricos gerais, que permeiam a discussão e servem de base para o objetivo específico da pesquisa.

\subsubsection{Corrente de dreno em função da sobretensão de condução}

Como visto nos capítulos 2 e 3, a corrente de dreno IDs permeia a discussão em diversos pontos, inclusive servindo de base para o cálculo e extração de outros parâmetros. Desta forma, um dos primeiros passos da investigação foi extrair IDs em função da sobretensão de condução $\left(\mathrm{V}_{\mathrm{GT}}=\mathrm{V}_{\mathrm{GS}}-\mathrm{V}_{\mathrm{TH}}\right)$, vide Figura 30 e Figura 31. A tensão de limiar será abordada na próxima seção.

Figura $30-\mathrm{I}_{\mathrm{DS}}$ versus $\mathrm{V}_{\mathrm{GT}}$ para $\mathrm{W}$ variável com $\mathrm{L}=100 \mathrm{~nm} @ \mathrm{~V}_{\mathrm{DS}}=750 \mathrm{mV}$

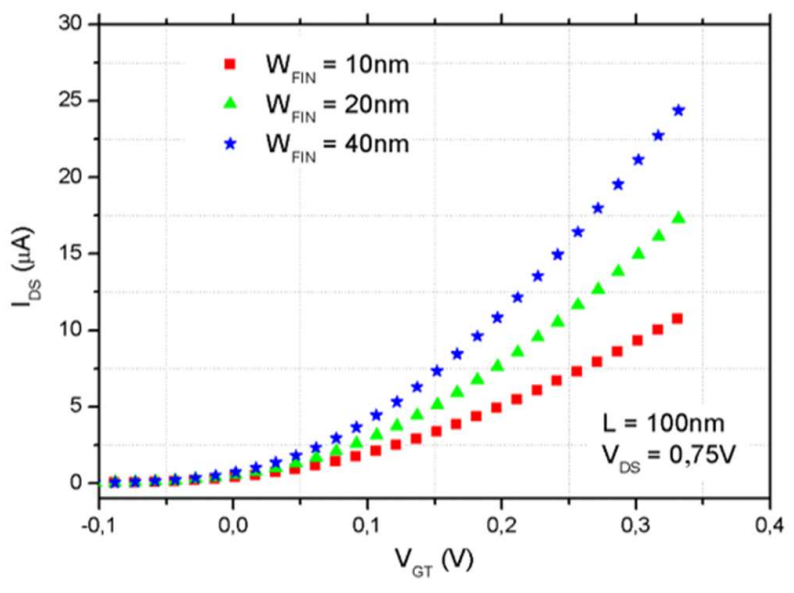

Fonte: Autor 
Figura 31 - IDs versus $V_{\text {GT }}$ para L variável com $\mathrm{W}_{\text {FIN }}=10 \mathrm{~nm} @ \mathrm{~V}_{\mathrm{DS}}=750 \mathrm{mV}$

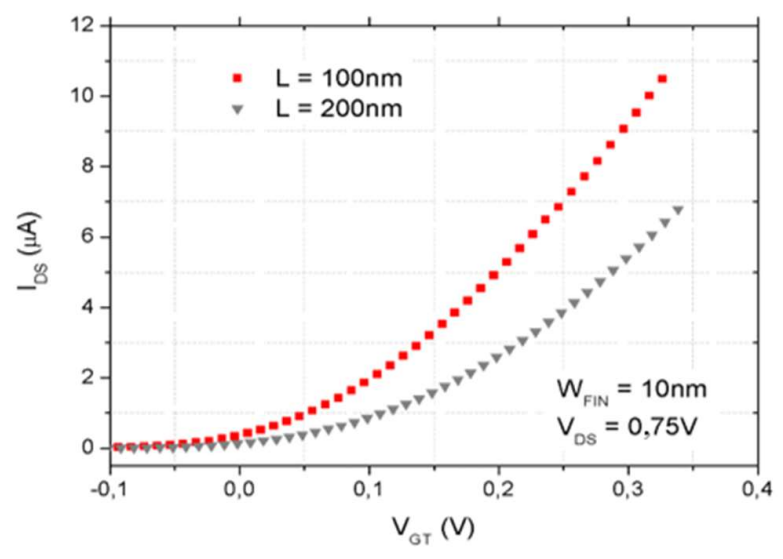

Fonte: Autor

É possível comprovar a existência da proporcionalidade $I_{D S} \propto \frac{W}{L}$, já que os maiores valores de corrente foram obtidos para transistores com larguras superiores e comprimentos de canal inferiores $\left(\mathrm{W}_{\mathrm{FIN}}=40 \mathrm{~nm} \mathrm{e} \mathrm{L}=100 \mathrm{~nm}\right)$, conforme era esperado na hipótese inicial.

\subsubsection{Tensão de Limiar (VTH) e Inclinação de Sublimiar (S)}

A sobretensão de condução leva em consideração a tensão de porta aplicada menos a tensão de limiar do dispositivo. A tensão de limiar pode ser obtida por diversas ferramentas, e no caso deste trabalho, foi extraída através do método da segunda derivada (CONDE et al., 2013). Este método consiste em analisar o ponto máximo da derivada de segunda ordem da curva $I_{D} \times V_{G}$ extraída experimentalmente (Figura 32 e eq. (46)).

Figura 32 - Segunda derivada de $\mathrm{I}_{\mathrm{D}} \times \mathrm{V}_{\mathrm{G}}$ de um transistor genérico

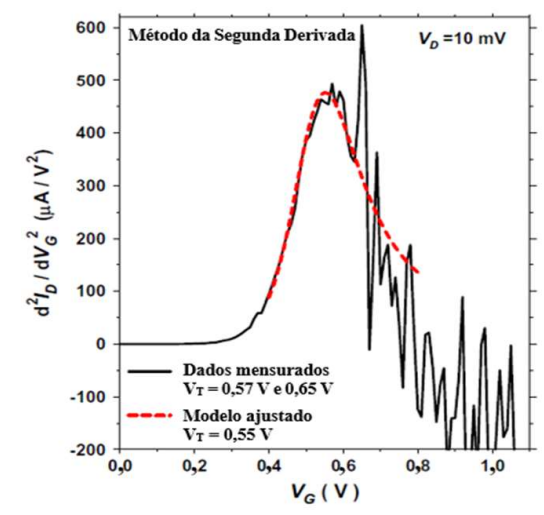

Fonte: Autor “adaptado de" Conde et al., 2013 


$$
V_{T H}=\max \left(\frac{\partial^{2} I_{D}}{\partial^{2} V_{G}}\right)
$$

Além disso, para a determinação da inclinação de sublimiar foi mensurada a tensão necessária para se variar uma década de corrente de dreno, remetendo diretamente à sua equação característica (eq. (19)). Houve variações nas tensões de limiar dos dispositivos, cujos extremos estavam compreendidos entre $647 \mathrm{mV}\left(\mathrm{W}_{\text {FIN }}=20 \mathrm{~nm}\right.$ e L $\left.=100 \mathrm{~nm}\right)$ e $701 \mathrm{mV}$ $\left(\mathrm{W}_{\mathrm{FIN}}=10 \mathrm{~nm}\right.$ e L $\left.=400 \mathrm{~nm}\right)$. Já a excursão da inclinação de sublimiar se deu entre 63,03 $\mathrm{mV} /$ déc $\left(\mathrm{W}_{\text {FIN }}=10 \mathrm{~nm}\right.$ e $\left.\mathrm{L}=200 \mathrm{~nm}\right)$ e $65,38 \mathrm{mV} /$ déc $\left(\mathrm{W}_{\text {FIN }}=10 \mathrm{~nm}\right.$ e $\left.\mathrm{L}=400 \mathrm{~nm}\right)$. A Tabela 1 e a Tabela 2 abrangem todos os dados, que estão graficamente representados na Figura 33. Os valores de $\mathrm{I}_{\mathrm{D} 1}, \mathrm{~V}_{\mathrm{G} 1}, \mathrm{I}_{\mathrm{D} 2}, \mathrm{~V}_{\mathrm{G} 2}$ foram utilizados para o cálculo de $\mathrm{S}$ (tensão necessária na variação de uma década de corrente).

Tabela $1-\mathrm{V}_{\mathrm{TH}}$ e S para diferentes $\mathrm{W}_{\mathrm{FIN}}$ com $\mathrm{L}=100 \mathrm{~nm} @ \mathrm{~V}_{\mathrm{DS}}=25 \mathrm{mV}$

\begin{tabular}{cccccccc}
\hline $\begin{array}{c}\mathrm{W}_{\mathrm{FIN}} \\
(\mathrm{nm})\end{array}$ & $\mathrm{V}_{\mathrm{DS}}(\mathrm{mV})$ & $\begin{array}{c}\mathrm{I}_{\mathrm{D} 1} \\
(\mathrm{nA})\end{array}$ & $\mathrm{V}_{\mathrm{G} 1}(\mathrm{mV})$ & $\begin{array}{c}\mathrm{I}_{\mathrm{D} 2} \\
(\mathrm{nA})\end{array}$ & $\begin{array}{c}\mathrm{V}_{\mathrm{G} 2}(\mathrm{mV}) \\
(\mathrm{mV})\end{array}$ & $\begin{array}{c}\mathrm{V}_{\mathrm{TH}} \\
(\mathrm{mV} / \text { déc })\end{array}$ \\
\hline 10 & 25 & 0,100 & 436,92 & 0,996 & 500,85 & 668,71 & 63,93 \\
\hline 15 & 25 & 0,101 & 419,90 & 1,00 & 484,92 & 650,68 & 65,01 \\
\hline 20 & 25 & 0,101 & 429,74 & 1,00 & 493,65 & 647,21 & 63,91 \\
\hline 40 & 25 & 0,101 & 406,58 & 1,00 & 470,96 & 663,36 & 64,38 \\
\hline
\end{tabular}

Fonte: Autor

Tabela 2 - $\mathrm{V}_{\mathrm{TH}}$ e S para diferentes L com W=10 nm @ $\mathrm{V}_{\mathrm{DS}}=25 \mathrm{mV}$

\begin{tabular}{cccccccc}
\hline $\mathrm{L}(\mathrm{nm})$ & $\mathrm{V}_{\mathrm{DS}}(\mathrm{mV})$ & $\begin{array}{c}\mathrm{I}_{\mathrm{D} 1} \\
(\mathrm{nA})\end{array}$ & $\mathrm{V}_{\mathrm{G} 1}(\mathrm{mV})$ & $\begin{array}{c}\mathrm{I}_{\mathrm{D} 2} \\
(\mathrm{nA})\end{array}$ & $\begin{array}{c}\mathrm{V}_{\mathrm{G} 2}(\mathrm{mV}) \\
(\mathrm{mV})\end{array}$ & $\begin{array}{c}\mathrm{V}_{\mathrm{TH}} \\
(\mathrm{mV} / \text { déc })\end{array}$ \\
\hline 100 & 25 & 0,100 & 436,92 & 0,996 & 500,85 & 668,71 & 63,93 \\
\hline 200 & 25 & 0,100 & 454,52 & 1,00 & 517,56 & 657,19 & 63,03 \\
\hline 400 & 25 & 0,100 & 513,10 & 1,00 & 578,48 & 701,02 & 65,38 \\
\hline
\end{tabular}

Fonte: Autor 
Figura 33 - Tensão $\mathrm{V}_{\mathrm{TH}}$ e Inclinação de Sublimiar S em função da variação de a) $W_{\text {FIN }}$ e b) $L$
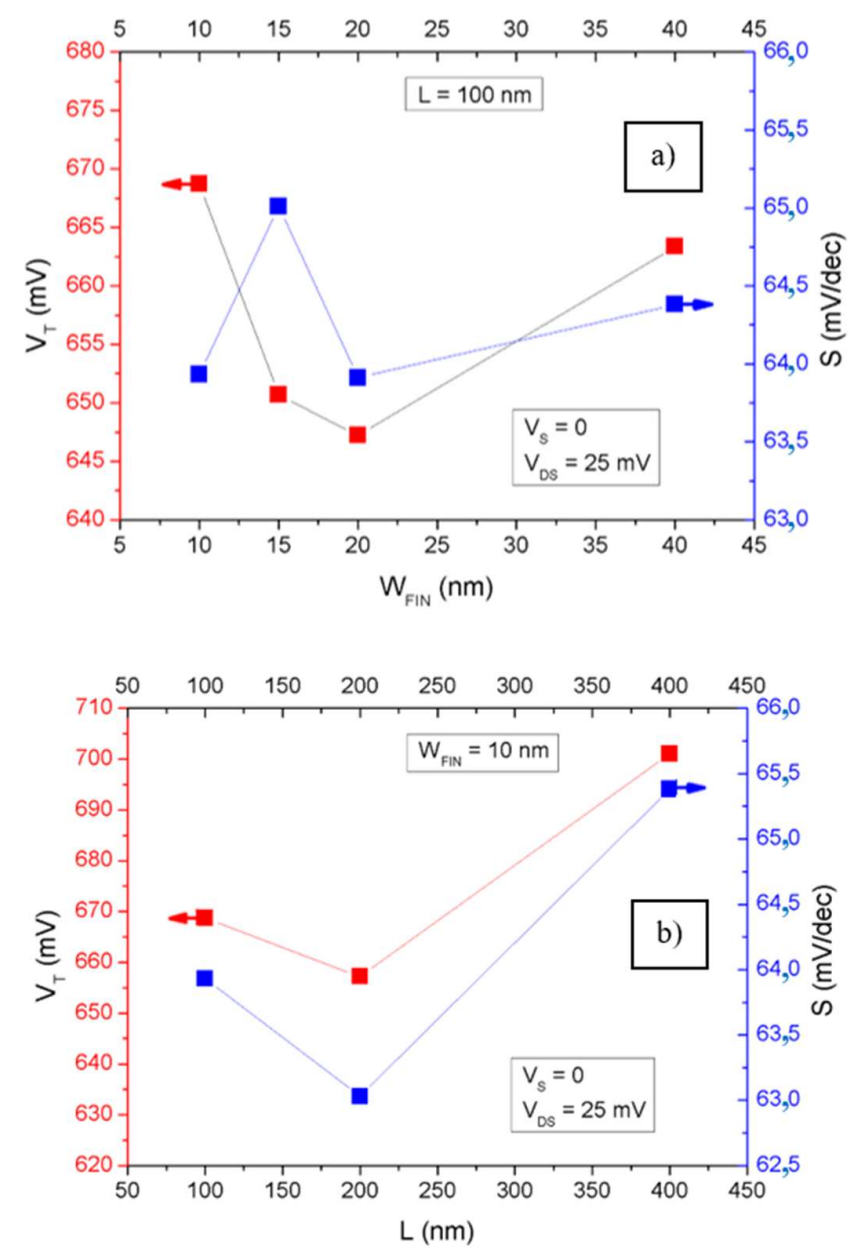

Fonte: Autor

Após comparar os dados acima com os da literatura, apresentados no capítulo 2, é possível notar uma ótima convergência entre os resultados, sendo que a tensão de limiar se mostrou ligeiramente maior e, em contra partida, a inclinação de sublimiar apresentou resultados menores e mais próximos do limite teórico, o que sugere que os nanofios empilhados possuem um bom fator de corpo $\alpha$.

\subsubsection{Corrente de dreno em função de VDS}

Outra relação extraída foi a corrente de dreno em função de $V_{D S}$, apresentado na Figura 34. Conforme esperado, as maiores correntes de dreno nas curvas foram alcançadas em transistores com maiores valores de $\mathrm{W}_{\mathrm{FIN}}$ e menores comprimentos de canal L. Além disso, 
confirma-se a relação entre aumento de corrente a partir da elevação da sobretensão de porta $\mathrm{V}_{\mathrm{GT}}$.

Figura 34 - $\mathrm{I}_{\mathrm{DS}}$ em função de $\mathrm{V}_{\mathrm{DS}}$ para $\mathrm{W}_{\mathrm{FIN}}$ variável e L variável em diferentes $\mathrm{V}_{\mathrm{GT}}$
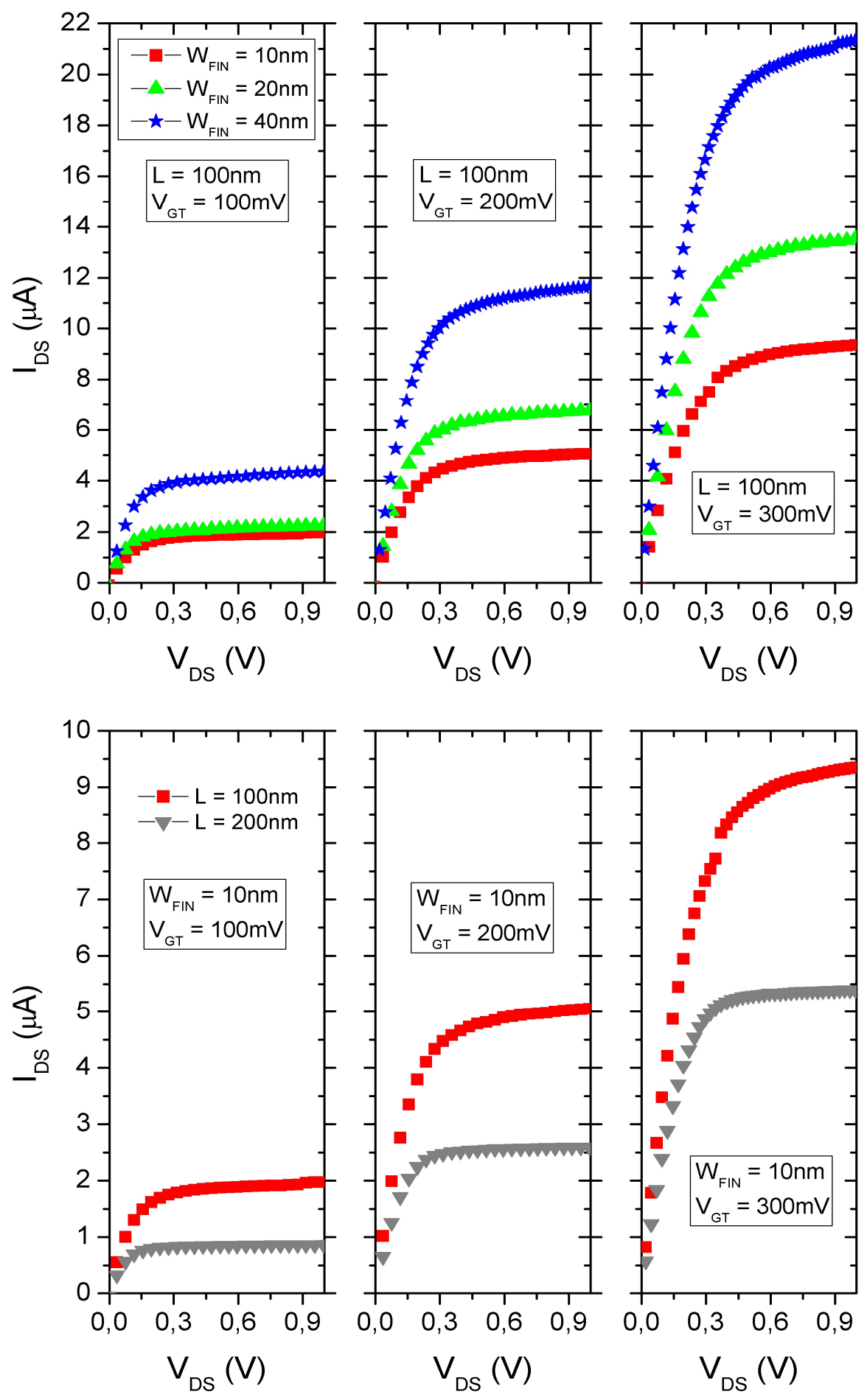

Fonte: Autor 


\subsubsection{Mobilidade de baixo campo e Degradação da Mobilidade}

A fim de detalhar melhor o comportamento do dispositivo, também foram extraídas a mobilidade de baixo campo $\left(\mu_{0}\right)$ e a degradação da mobilidade $(\theta)$. Para tanto, foram utilizados os métodos relatados em (GHIBAUDO, 1988), que demonstra e valida as seguintes equações:

$$
\begin{aligned}
\frac{I_{D}}{g_{m}^{1 / 2}} & =\left(\frac{W}{L} C_{O X} \mu_{0} V_{D}\right)^{\frac{1}{2}}\left(V_{G}-V_{T H}\right) \\
\theta & =\frac{\left(\frac{I_{D}}{\left[g_{m}\left(V_{G}-V_{T H}\right)\right]}-1\right)}{\left(V_{G}-V_{T H}\right)}
\end{aligned}
$$

A Figura 35 abaixo apresenta os resultados de $\mu_{0}$ e $\theta$ para os dispositivos:

Figura $35-\mu_{0}$ e $\theta$ em função de $\mathrm{W}_{\mathrm{FIN}}$ variável com L $=100 \mathrm{~nm} @ \mathrm{~V}_{\mathrm{DS}}=25 \mathrm{mV}$

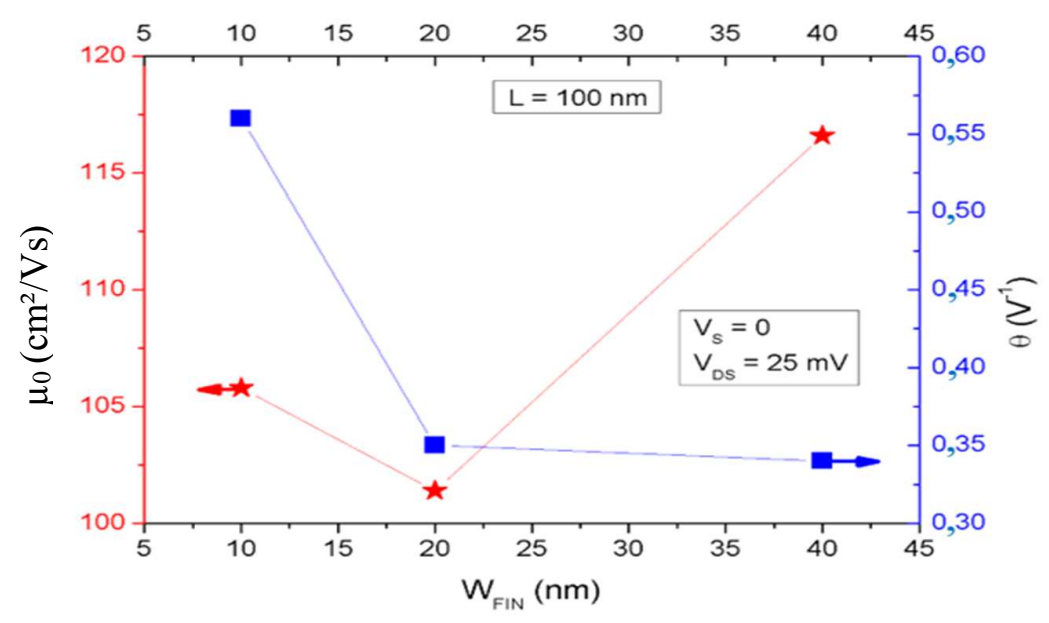

Fonte: Autor

O melhor resultado de mobilidade de baixo campo foi obtido para o $\mathrm{W}_{\mathrm{FIN}}=40 \mathrm{~nm}$, embora os três dispositivos apresentem valores muito próximos entre si, com uma pequena variação de $15,2 \mathrm{~cm}^{2} / \mathrm{V}_{\mathrm{S}}$ entre os extremos. Também não foi observada grande variação na degradação de mobilidade, sendo o nanofio de $\mathrm{W}_{\mathrm{FIN}}=40 \mathrm{~nm}$ aquele que possui o melhor desempenho, com $\theta=0,34 \mathrm{~V}^{-1}$. A Tabela 3 abaixo também apresenta os valores de resistência série $\left(\mathrm{R}_{\mathrm{S}} \mathrm{W}\right)$ para os nanofios empilhados, que foi extraído conforme método apresentado em (DIXIT et al., 2005). 
Tabela 3 - Resultados de $\mu_{0}, \theta$ e $\mathrm{R}_{\mathrm{S}} \mathrm{W}$ para diferentes $\mathrm{W}_{\mathrm{FIN}}$ de nanofios empilhados

\begin{tabular}{cccc}
\hline \multicolumn{4}{c}{ Nanofio Empilhado $(\mathrm{L}=100 \mathrm{~nm})$} \\
\hline $\begin{array}{c}\mathrm{W}_{\mathrm{FIN}} \\
(\mathrm{nm})\end{array}$ & $\begin{array}{c}\mu_{0} \\
\left(\mathrm{~cm}^{2} / \mathrm{Vs}\right)\end{array}$ & $\theta\left(\mathrm{V}^{-1}\right)$ & $\begin{array}{c}\mathrm{R}_{\mathrm{s}} \mathrm{W} \\
\left(\Omega^{*} \mu \mathrm{m}\right)\end{array}$ \\
\hline 10 & 105,8 & 0,56 & 1391 \\
\hline 20 & 101,4 & 0,35 & 1229 \\
\hline 40 & 116,6 & 0,34 & 1413 \\
\hline
\end{tabular}

Fonte: Autor

\subsection{RESULTADOS DE NÃO LINEARIDADE PARA OS TRANSISTORES NANOFIOS EMPILHADOS}

Em posse dos dados anteriores, é possível aprofundar a investigação em direção ao descobrimento do comportamento não linear dos transistores nanofios empilhados. Em suma, quer-se observar os resultados de distorção dos harmônicos pares e ímpares, e isto será feito sob diversos prismas, a fim de elucidar e explicar o fenômeno e suas relações. Tal análise já foi publicada em artigo na Journal of Integrated Circuits and Systems (JICS), conforme consta no Anexo B.

\subsubsection{Distorção Harmônica em função de $V_{G T}$}

A partir das curvas $I_{D} \times V_{G}$ extraídas do analisador de sinais e utilizando-se do algoritmo implementado no MathCAD com o método da função integral (IFM apresentado no capítulo 4), foi possível extrair a distorção harmônica total e a não linearidade proveniente do harmônico de terceira ordem. Seus dados foram mostrados em função de $\mathrm{V}_{\mathrm{GT}}$ a fim de se observar a tendência da curva e a influência da tensão de porta. O IFM foi empregado considerando uma amplitude de tensão $\mathrm{V}_{\mathrm{A}}=50 \mathrm{mV}$. 
Figura 36 - Resultados de THD e HD3 em função de $V_{\text {GT }}$ para $\mathrm{W}_{\mathrm{FIN}}$ variável com $\mathrm{L}=100 \mathrm{~nm} @ \mathrm{~V}_{\mathrm{DS}}=750 \mathrm{mV}$

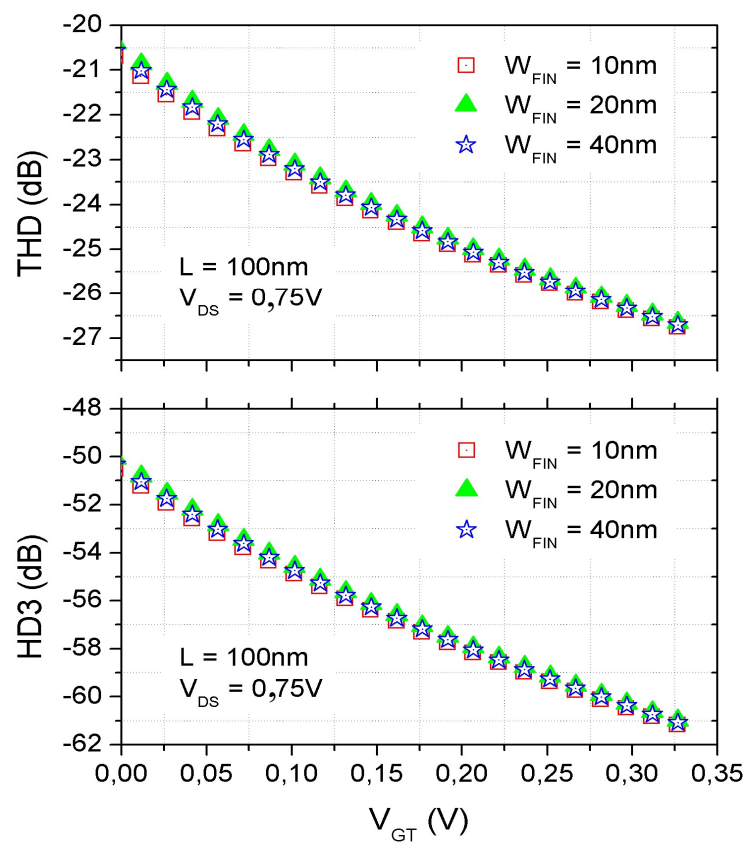

Fonte: Autor

Figura 37 - Resultados de THD e HD3 em função de $V_{\text {GT }}$ para $\mathrm{L}$ variável com $\mathrm{W}=10 \mathrm{~nm} @ \mathrm{~V}_{\mathrm{DS}}=750 \mathrm{mV}$

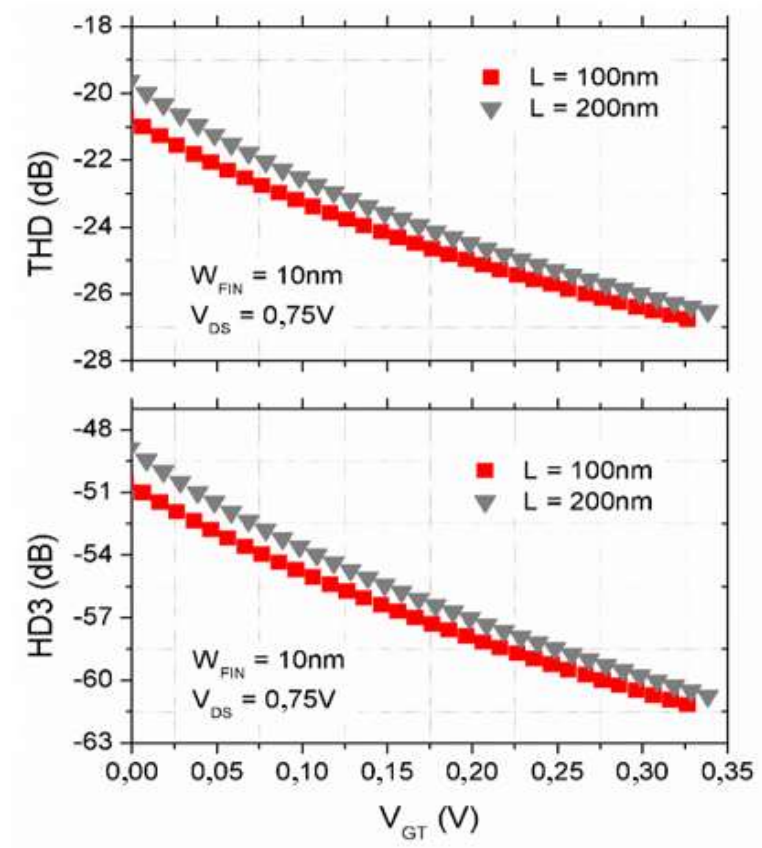

Fonte: Autor 
O primeiro ponto a ser observado é que, nos transistores nanofios empilhados, o harmônico de segunda ordem (HD2) é o elemento preponderante causador de distorção, uma vez que as magnitudes de THD e HD3 são muito distantes entre si: enquanto THD varia entre $-20 \mathrm{~dB}$ e $-27 \mathrm{~dB}$, HD3 abrange apenas uma pequena parcela da não linearidade, variando entre $-50 \mathrm{~dB}$ e $-61 \mathrm{~dB}$. Este comportamento vai ao encontro do que foi observado para os transistores nanofios de nível único em (PAZ et al., 2016).

O gráfico da Figura 36 mostra que, para toda aplicação que exige um amplificador operacional com distorção harmônica inferior à $-20 \mathrm{~dB}$, qualquer um dos nanofios empilhados analisados poderiam ser empregados. Entretanto, o gráfico não permite observar grande discrepância entre as distorções dos diferentes dispositivos, haja visto que as curvas de todos os $\mathrm{W}_{\mathrm{FIN}}$ estão sobrepostas, tanto em THD quando em HD3. Isto faz com que não se possa, por ora, chegar a uma conclusão a respeito da relação entre a largura de canal e a não linearidade do dispositivo. Porém, é possível notar na Figura 37 que o transistor com maior comprimento de canal apresentou maior distorção harmônica. Na intenção de encontrar validação matemática para este resultado físico, é preciso introduzir uma nova equação para HD2, uma vez que àquela apresentada na eq. (33) está descrita principalmente em função da transcondutância, cujo resultado ainda não foi apresentado. Em (DORIA et al., 2010), mostra-se que a equação de HD2 pode ser reescrita conforme a eq. (49):

$$
H D 2=\frac{V_{A}}{V_{G T}\left[2+3\left(\theta+R_{S} K_{\text {low }}\right) V_{G T}+\left(\theta+R_{S} K_{\text {low }}\right)^{2} V_{G T}{ }^{2}\right]}
$$

Logo, é possível verificar que a trajetória das curvas dos resultados experimentais está de acordo com a equação teórica, uma vez que ambos indicam uma relação inversamente proporcional entre distorção harmônica de segunda ordem e sobretensão de porta $\left(\uparrow V_{\mathrm{GT}} \rightarrow \downarrow \mathrm{HD} 2\right)$. A fim de explicitar a relação entre não linearidade e as dimensões do componente, pode-se reescrever o ganho $\mathrm{K}_{\text {low }}$ como:

$$
K_{\text {low }}=\frac{\mu_{0}}{1+\theta V_{G T}} C_{O X}\left(\frac{W_{(S T A C K E D)}}{L}\right)
$$

As duas últimas equações explicam o comportamento da distorção na Figura 37, já que para um maior comprimento de canal $\mathrm{L}$, tem-se um menor $\mathrm{K}_{\text {low }}$, e quanto menor $\mathrm{K}_{\text {low }}$, maior $\mathrm{HD} 2$, justificando o melhor resultado do transistor nanofio com $\mathrm{L}=100 \mathrm{~nm}$ em relação ao com 
$\mathrm{L}=200 \mathrm{~nm}$. Além disso, os dados da Tabela 3 sugerem que os demais fatores influenciadores de HD2, que estão no denominador da eq. (49) ( $\theta$ e $\left.\mathrm{R}_{S} \mathrm{~K}_{\text {low }}\right)$, não possuem grande variação de magnitude para cada $\mathrm{W}_{\mathrm{FIN}}$, de modo que todos os termos fornecem sua contribuição para HD2, sem uma dominância individual aparente.

\subsubsection{Distorção Harmônica em função de $\mathbf{g}_{\mathrm{m}} / \mathrm{IDS}_{\mathrm{DS}}$}

Uma vez que não foi possível verificar a relação entre $\mathrm{W}_{\text {FIN }}$ e HD2, faz-se necessário empregar outra análise: observar a distorção harmônica dos dispositivos em função de $\mathrm{g}_{\mathrm{m}} / \mathrm{I}_{\mathrm{DS}}$. Esta estratégia permite investigar os resultados com todos os dispositivos polarizados na mesma região operacional, excluindo-se a dependência da sobretensão de condução. Para tanto, primeiro é preciso extrair as transcondutâncias dos nanofios empilhados. Os gráficos da Figura 38 e Figura 39 expõe os resultados de $g_{m}$ em função de $V_{\text {GT }}$ para os diferentes nanofios. Eles foram calculados a partir da derivada de $\mathrm{I}_{\mathrm{D}} \mathrm{x} \mathrm{V}_{\mathrm{GT}}$, em concordância com a eq. (1). Adicionalmente, a Tabela 4 frisa alguns dos resultados, apresentando também os valores da condutância de saída $\mathrm{g}_{\mathrm{D}}$, que foram calculados através da derivada de $\mathrm{I}_{\mathrm{DS}} \mathrm{x} \mathrm{V}_{\mathrm{DS}}$, conforma mostra a eq. (31).

Figura 38 - $\mathrm{g}_{\mathrm{m}}$ em função de $\mathrm{V}_{\mathrm{GT}}$ para $\mathrm{W}_{\mathrm{FIN}}$ variável

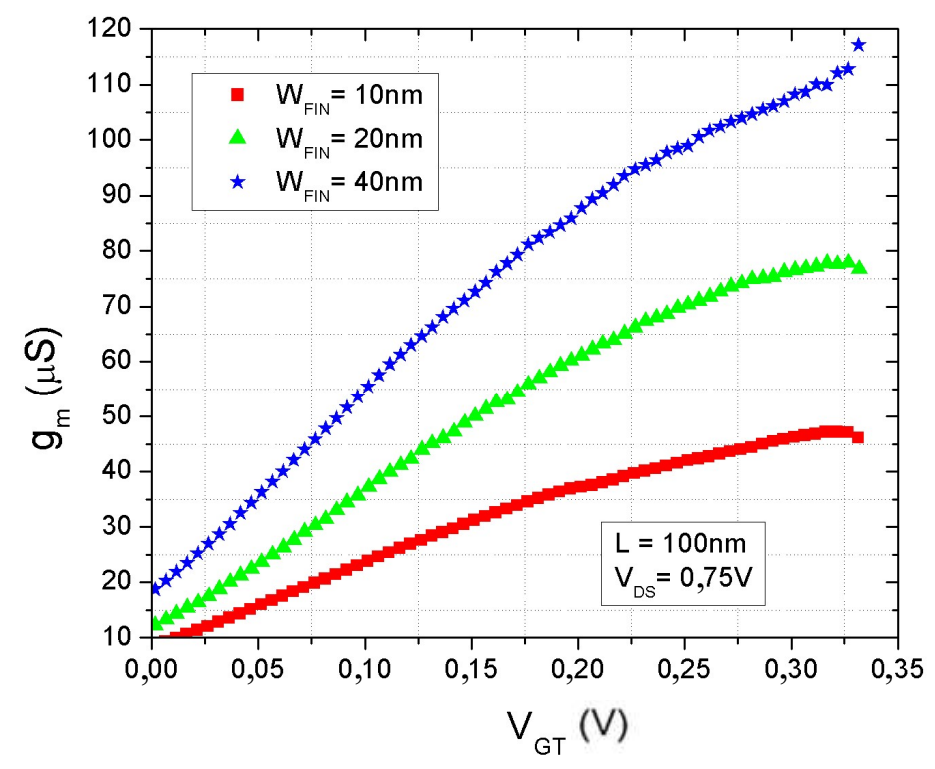

Fonte: Autor 
Figura $39-\mathrm{g}_{\mathrm{m}}$ em função de $\mathrm{V}_{\mathrm{GT}}$ para $\mathrm{L}$ variável

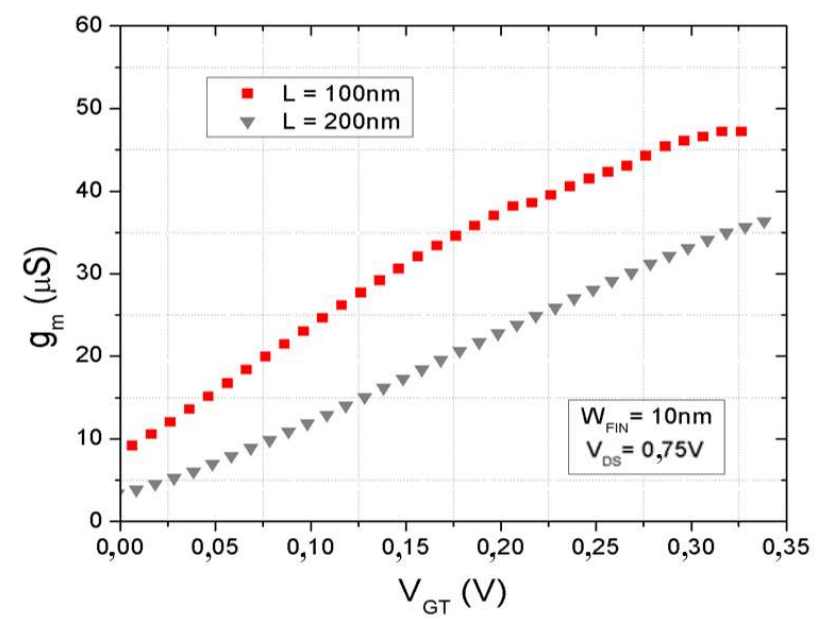

Fonte: Autor

Tabela 4 - Resultados experimentais de $g_{m}$ e $g_{D}$ em função de $V_{G T}$ para $W_{\text {Fin }}$ e L variáveis

\begin{tabular}{|c|c|c|c|}
\hline $\begin{array}{l}\text { W FIN }_{\text {f }} \\
(\mathrm{nm})\end{array}$ & $\mathrm{g}_{\mathrm{m}} @ \mathrm{~V}_{\mathrm{GT}}=100 \mathrm{mV}(\mu \mathrm{S})$ & $\mathrm{g}_{\mathrm{m}} @ \mathrm{~V}_{\mathrm{GT}}=200 \mathrm{mV}(\mu \mathrm{S})$ & $\mathrm{g}_{\mathrm{m}} @ \mathrm{~V}_{\mathrm{GT}}=300 \mathrm{mV}(\mu \mathrm{S})$ \\
\hline 10 & 23,85 & 37,27 & 46,25 \\
\hline 20 & 37,24 & 61,18 & 76,61 \\
\hline 40 & 55,51 & 87,69 & 108,21 \\
\hline $\begin{array}{l}\mathrm{W}_{\mathrm{FIN}} \\
(\mathrm{nm})\end{array}$ & $\mathrm{g}_{\mathrm{D}} @ \mathrm{~V}_{\mathrm{GT}}=100 \mathrm{mV}(\mu \mathrm{S})$ & $\mathrm{g}_{\mathrm{D}} @ \mathrm{~V}_{\mathrm{GT}}=200 \mathrm{mV}(\mu \mathrm{S})$ & $\mathrm{g}_{\mathrm{D}} @ \mathrm{~V}_{\mathrm{GT}}=300 \mathrm{mV}(\mu \mathrm{S})$ \\
\hline 10 & 0,20 & 0,48 & 1,12 \\
\hline 20 & 0,33 & 0,77 & 1,78 \\
\hline 40 & 0,69 & 1,42 & 2,69 \\
\hline $\begin{array}{c}\mathrm{L} \\
(\mathrm{nm})\end{array}$ & $\mathrm{g}_{\mathrm{m}} @ \mathrm{~V}_{\mathrm{GT}}=100 \mathrm{mV}(\mu \mathrm{S})$ & $\mathrm{g}_{\mathrm{m}} @ \mathrm{~V}_{\mathrm{GT}}=200 \mathrm{mV}(\mu \mathrm{S})$ & $\mathrm{g}_{\mathrm{m}} @ \mathrm{~V}_{\mathrm{GT}}=300 \mathrm{mV}(\mu \mathrm{S})$ \\
\hline 100 & 23,82 & 37,63 & 46,25 \\
\hline 200 & 12,42 & 23,31 & 33,12 \\
\hline $\begin{array}{c}\mathrm{L} \\
(\mathrm{nm})\end{array}$ & $\mathrm{g}_{\mathrm{D}} @ \mathrm{~V}_{\mathrm{GT}}=100 \mathrm{mV}(\mu \mathrm{S})$ & $\mathrm{g}_{\mathrm{D}} @ \mathrm{~V}_{\mathrm{GT}}=200 \mathrm{mV}(\mu \mathrm{S})$ & $\mathrm{g}_{\mathrm{D}} @ \mathrm{~V}_{\mathrm{GT}}=300 \mathrm{mV}(\mu \mathrm{S})$ \\
\hline 100 & 0,20 & 0,48 & 1,12 \\
\hline 200 & 0,03 & 0,17 & 0,61 \\
\hline
\end{tabular}

Fonte: Autor 
A partir dos valores de $\mathrm{g}_{\mathrm{m}}$ calculados, foi possível realizar a nova análise proposta. À primeira vista, nota-se a relação inversa entre a curva de THD $\times g_{m} / I_{D}$ em vista de THD $\mathrm{x}$ $\mathrm{V}_{\mathrm{GT}}$, ou seja, nesta análise, a distorção aumenta com o acréscimo de $\mathrm{g}_{\mathrm{m}} / \mathrm{I}_{\mathrm{DS}}$, ao passo que antes diminuía com o aumento de $\mathrm{V}_{\mathrm{GT}}$. Este comportamento está de acordo com os resultados do nanofio de porta tripla (PAZ et al., 2016) cuja trajetória das curvas eram as mesmas, e da eq. (49).

Figura 40 - Resultados de THD e HD3 em função de $g_{m} / I_{D s}$ para $\mathrm{W}_{\text {FIN }}$ variável com L = $100 \mathrm{~nm} @ \mathrm{~V}_{\mathrm{DS}}=750 \mathrm{mV}$
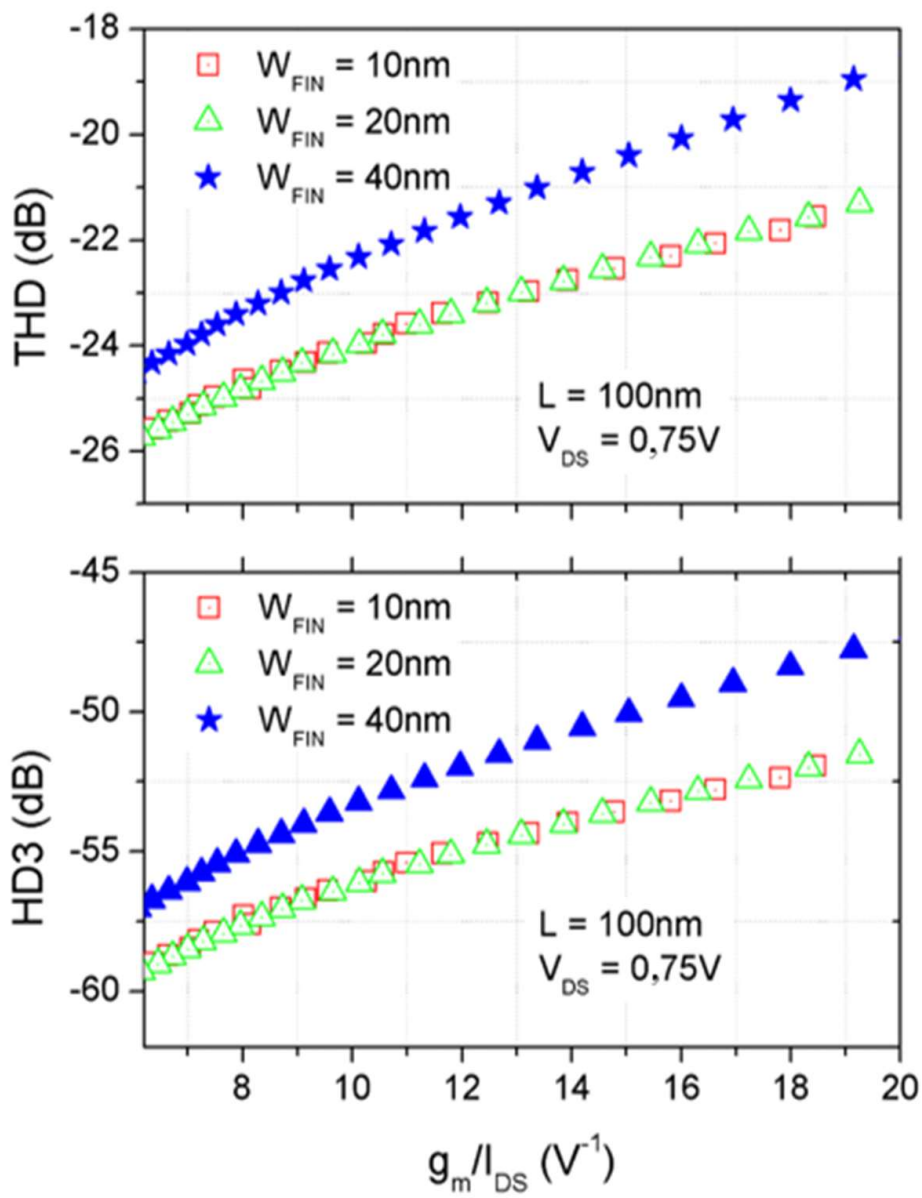

Fonte: Autor

Embora os dados de distorção ainda estejam próximos entre si, pela primeira vez observou-se uma dependência entre THD e a largura de canal do dispositivo. A Figura 40 sugere que os nanofios empilhados com maiores $W_{\text {FIN }}$ apresentam maiores distorções harmônicas, porém o resultado não é totalmente conclusivo já que $\mathrm{W}_{\text {FIN }}=10 \mathrm{~nm}$ e $W_{\text {FIN }}=20 \mathrm{~nm}$ traduzem valores de distorção muito semelhantes. 
Figura 41 - Resultados de THD e HD3 em função de $g_{m} / I_{D S}$ para $L$ variável com $\mathrm{W}=10 \mathrm{~nm} @ \mathrm{~V}_{\mathrm{DS}}=750 \mathrm{mV}$
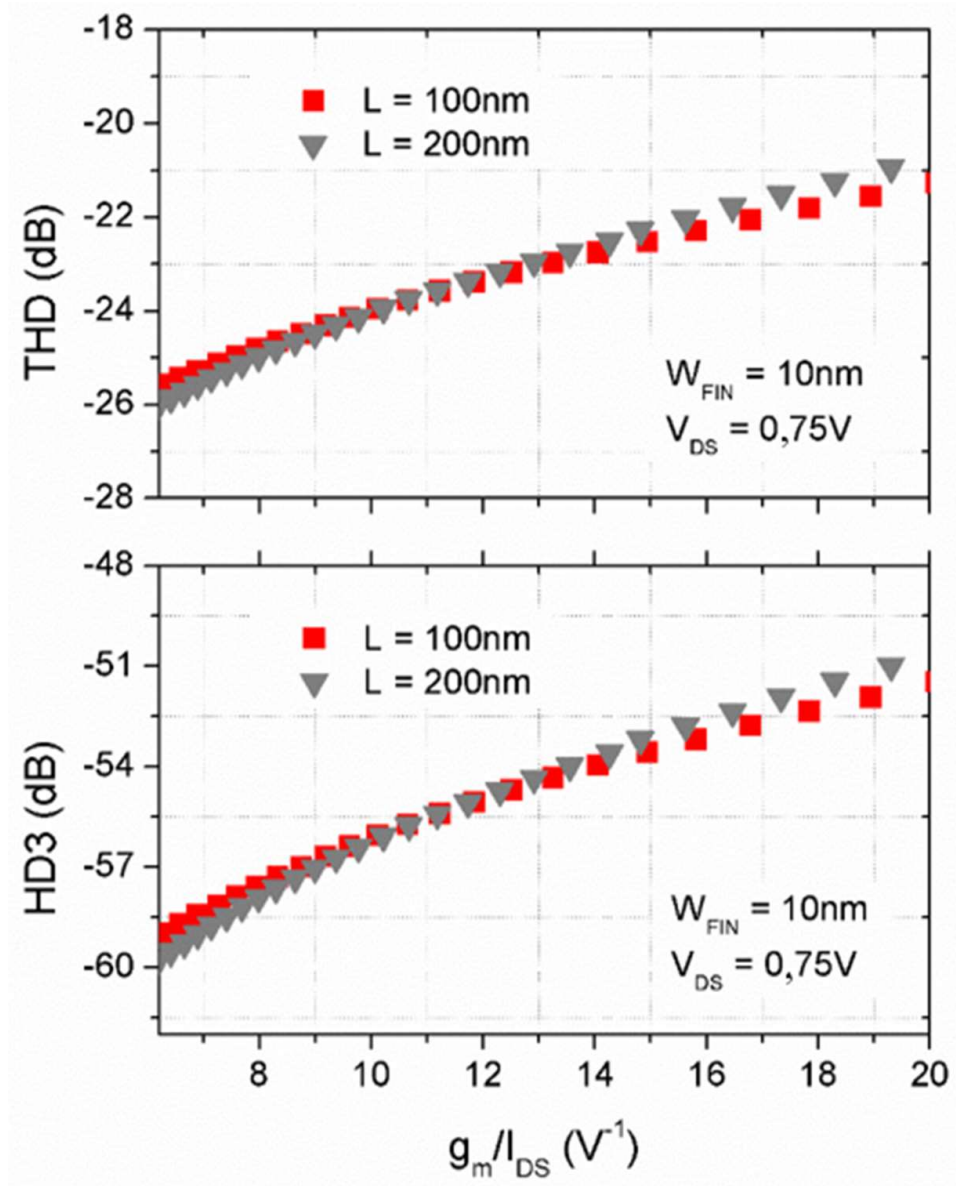

Fonte: Autor

Para estes transistores com comprimento de canal variável polarizados em um mesmo $\mathrm{g}_{\mathrm{m}} / \mathrm{I}_{\mathrm{DS}}$, nota-se que a distorção harmônica total e a distorção harmônica de terceira ordem são pouco dependentes de L, já que, nesta visualização, a variação de L não se mostrou relevante para a separação dos dados.

\subsubsection{Distorção Harmônica dividida pelo Ganho de Tensão em função de $g_{m} / I_{D S}$}

A próxima perspectiva de análise leva em consideração a influência do ganho Av, que foi calculado conforme a eq. (30), utilizando os dados da Tabela 4. Os resultados dos cálculos de Av podem ser encontrados na Tabela 5 e Tabela 6, e graficamente na Figura 42 e Figura 43. 
Tabela 5 - Resultados de $\mathrm{V}_{\mathrm{GT}}, \mathrm{g}_{\mathrm{m}} / \mathrm{I}_{\mathrm{DS}}$ e Av para os diferentes $\mathrm{W}_{\mathrm{FIN}}$ dos nanofios empilhados

\begin{tabular}{cccc}
\hline $\mathrm{W}_{\mathrm{FIN}}(\mathrm{nm})$ & $\mathrm{V}_{\mathrm{GT}}(\mathrm{mV})$ & $\mathrm{g}_{\mathrm{m}} / \mathrm{I}_{\mathrm{DS}}\left(\mathrm{V}^{-1}\right)$ & $\left.\mathrm{Av}_{\mathrm{dB}}\right)$ \\
\hline \multirow{2}{*}{10} & 100 & 12,04 & 41,34 \\
& 200 & 7,53 & 37,83 \\
& 300 & 5,00 & 32,33 \\
\hline \multirow{2}{*}{20} & 100 & 13,02 & 41,09 \\
& 200 & 7,91 & 37,94 \\
& 300 & 5,17 & 32,67 \\
\hline \multirow{2}{*}{40} & 100 & 12,70 & 38,08 \\
& 200 & 8,06 & 35,83 \\
& 300 & 5,12 & 32,09 \\
\hline
\end{tabular}

Fonte: Autor

Tabela 6 - Resultados de $\mathrm{V}_{\mathrm{GT}}, \mathrm{g}_{\mathrm{m}} / \mathrm{I}_{\mathrm{DS}}$ e $\mathrm{Av}$ para os diferentes $\mathrm{L}$ dos nanofios empilhados

\begin{tabular}{cccc}
\hline $\mathrm{L}(\mathrm{nm})$ & $\mathrm{V}_{\mathrm{GT}}(\mathrm{mV})$ & $\mathrm{g}_{\mathrm{m}} / \mathrm{I}_{\mathrm{DS}}\left(\mathrm{V}^{-1}\right)$ & $\mathrm{Av}_{\mathrm{v}}(\mathrm{dB})$ \\
\hline \multirow{2}{*}{100} & 100 & 12,04 & 41,34 \\
& 200 & 7,53 & 37,83 \\
& 300 & 5,00 & 32,33 \\
\hline \multirow{2}{*}{200} & 100 & 13,68 & 53,55 \\
& 200 & 8,79 & 42,60 \\
& 300 & 6,21 & 34,75 \\
\hline
\end{tabular}

Fonte: Autor

Figura 42 - Resultados de $A_{V}$ em função de $g_{m} / I_{D S}$ para $W_{\text {FIN }}$ variável e $\mathrm{L}=100 \mathrm{~nm} @ \mathrm{~V}_{\mathrm{DS}}=750 \mathrm{mV}$

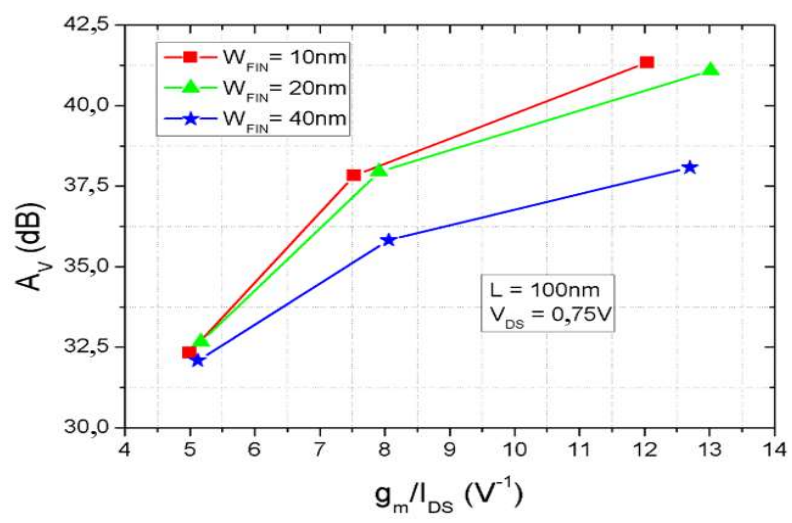

Fonte: Autor 
Figura 43 - Resultados de Av em função de $\mathrm{g}_{\mathrm{m}} / \mathrm{I}_{\mathrm{DS}}$ para L variável e $\mathrm{W}_{\mathrm{FIN}}=10 \mathrm{~nm} @ \mathrm{~V}_{\mathrm{DS}}=750 \mathrm{mV}$

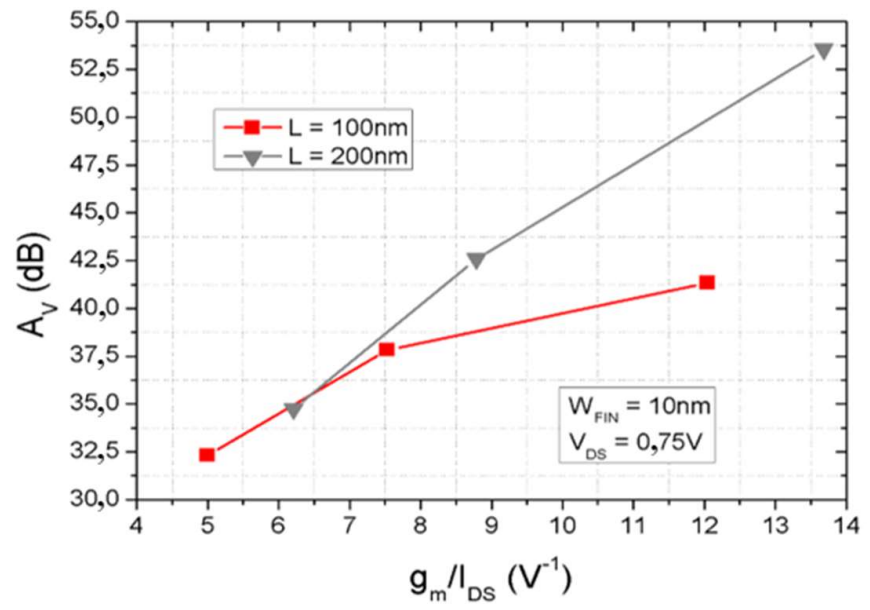

Fonte: Autor

Em todos os dispositivos, o ganho do transistor aumentou com o incremento de $\mathrm{g}_{\mathrm{m}} / \mathrm{I}_{\mathrm{DS}}$. A seção 5.2.2. explicitou a relação inversamente proporcional entre $g_{m} / I_{D S}$ e $V_{G T}$, e portanto pode-se atestar que $A_{V}$ decresce com o aumento da sobretensão de porta. Esse comportamento já era esperado, uma vez que o incremento de $\mathrm{V}_{\mathrm{GT}}$ significa que o nanofio transita da inversão moderada para a inversão forte, o que de fato deveria resultar em diminuição de seu ganho, conforme demonstrado em (SILVEIRA; FLANDRE; JESPERS, 1996).

Em posse destes novos dados de ganho de tensão, a próxima abordagem procura destacar as diferenças da não linearidade entre os dispositivos considerando seus diferentes ganhos. Para tanto, as distorções foram novamente extraídas, porém dividindo seus valores pelos respectivos Av de cada dispositivo, vide Figura 44 e Figura 45.

Comparando com a Figura 40 e a Figura 41, a trajetória das curvas mudou em decorrência dos baixos valores de Av, porém este método tornou os resultados mais discretos, separando os pontos e permitindo melhor avaliação da característica analógica do nanofio empilhado. Em acordo com o resultado da análise anterior (seção 5.2.2), o nanofio com maior $\mathrm{W}_{\text {FIN }}$ se mostrou aquele que possui a maior distorção harmônica entre todos. Entretanto, observando o gráfico para os transistores com L variados, à primeira vista o comportamento pode parecer inverso àquele relatado na Figura 37, já que o componente com $L=100 \mathrm{~nm}$ parece distorcer mais. Esta, porém, é uma constatação equivocada. A explicação para essa aparente inversão de desempenho está na Figura 43, que mostra que há uma grande diferença entre os ganhos dos dispositivos, resultando na mudança. O mesmo não ocorre para os $\mathrm{W}_{\text {FIN variados }}$ pois a variação máxima de Av está na ordem de 8,6\%, contra 29,5\% para os L variados. 
Figura 44 - Resultados de THD/Av e HD3/Av em função de $g_{m} / I_{D S}$ para $\mathrm{W}_{\text {FIN }}$ variável com $\mathrm{L}=100 \mathrm{~nm} @ \mathrm{~V}_{\mathrm{DS}}=750 \mathrm{mV}$
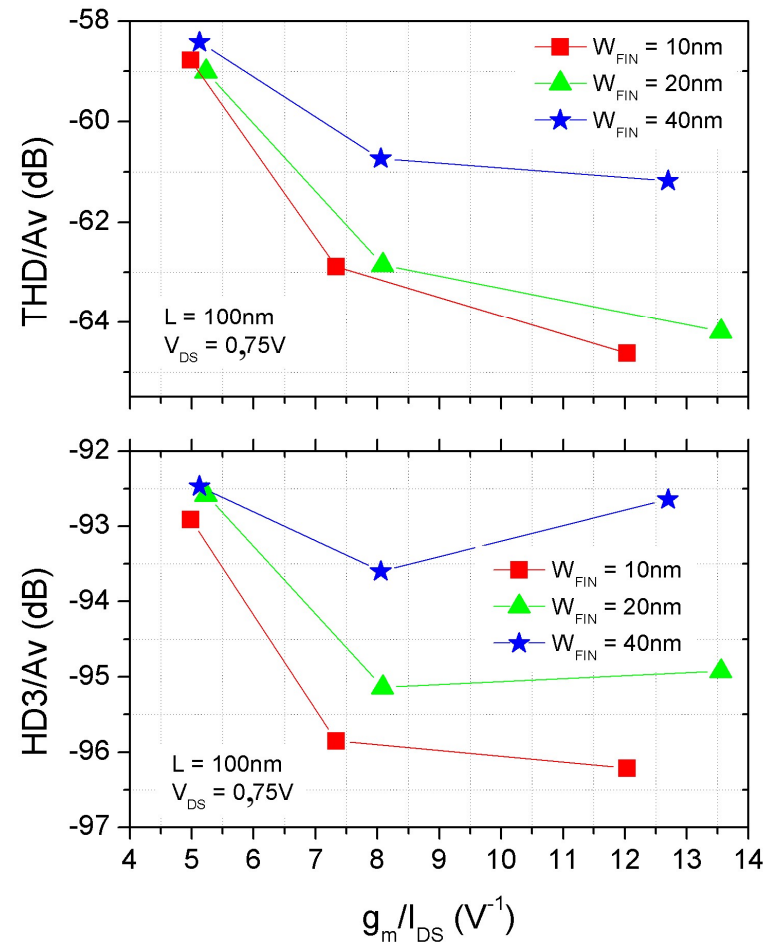

Fonte: Autor

Figura 45 - Resultados de THD/Av e HD3/Av em função de $g_{m} / I_{D S}$ para $\mathrm{L}$ variável com $\mathrm{W}=10 \mathrm{~nm} @ \mathrm{~V}_{\mathrm{DS}}=750 \mathrm{mV}$

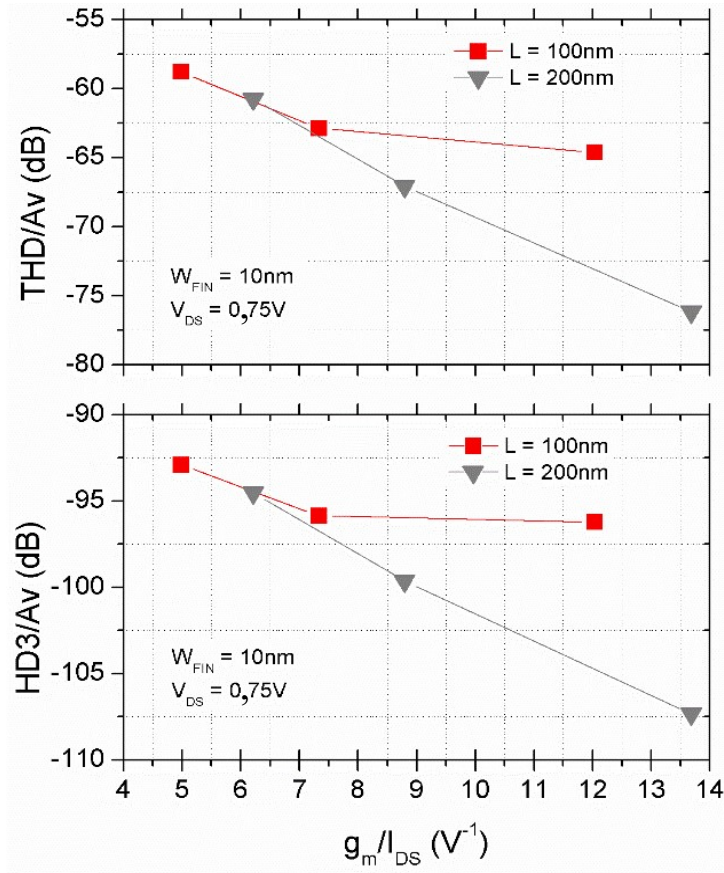

Fonte: Autor 


\subsubsection{Variação da transcondutância em função de $g_{\mathrm{m}} / \mathrm{IDS}$}

Finalmente, com o objetivo de confirmar as relações de não linearidade em função de $\mathrm{W}_{\mathrm{FIN}}$, faz-se uso novamente da eq. (33). Ela demonstra que a distorção harmônica de segunda ordem (que se mostrou majoritária em todos os resultados apresentados) é diretamente proporcional à $\partial g_{m} / \partial V_{G T}$. Desta forma, compilou-se $\partial g_{m} / \partial V_{G T}$ em função de $g_{m} / I_{D S}$ para os diferentes valores de $\mathrm{W}_{\mathrm{FIN}}$, a fim de realizar tal avaliação:

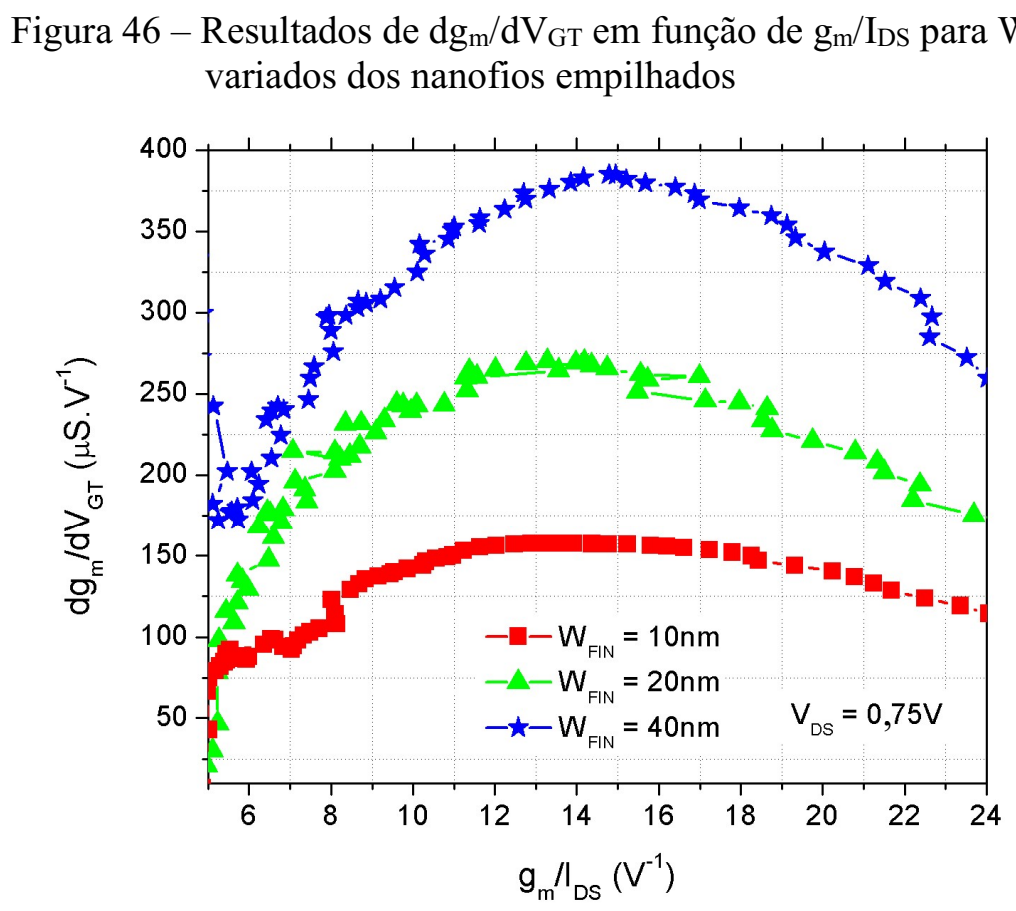

Fonte: Autor

Fica evidente que a maior variação de $\partial g_{m} / \partial V_{G T}$ é proveniente do nanofio com $\mathrm{W}_{\text {FIN }}=$ $40 \mathrm{~nm}$, sugerindo que ele possui a maior distorção harmônica total, o que converge com todos os resultados anteriores. Além disso, tal relação também foi observada para o nanofio de único nível em (PAZ et al., 2016), onde o transistor de porta tripla com maior W WIN também apresentou maior $\partial g_{m} / \partial V_{G T}$ e maior distorção harmônica. 


\subsection{NANOFIOS EMPILHADOS MULTIFINS E DE COMPRIMENTOS VARIADOS}

Até o momento, todas as análises levam a crer que, para os transistores nanofios empilhados, maiores dimensões tanto em largura quanto em comprimento resultam em dispositivos com maiores distorções harmônicas. A fỉm de se comprovar esta afirmação, um novo conjunto de dados foi extraído, desta vez de transistores nanofios empilhados multifins tipo nMOS com L fixo de $100 \mathrm{~nm}$ e $W_{\text {FIN }}$ variável de $10 \mathrm{~nm}, 25 \mathrm{~nm}$ e $60 \mathrm{~nm}$, contendo 10 canais cada. A análise voltada para os transistores multifins é um importante elemento para a comprovação da tendência que se está estabelecendo, já que nesta situação os gráficos se referem a uma média de resultados de diversos nanofios empilhados em paralelo, deixando as curvas menos suscetíveis às oscilações singulares e pontuais, o que traz um maior grau de confiança à conclusão. Além disso, foi realizada a extração de novos componentes com W FIN fixo em $10 \mathrm{~nm}$ e L variável de $120 \mathrm{~nm}$ e $400 \mathrm{~nm}$. Esta nova base de dados é proveniente do mesmo fabricante, nas mesmas condições de fabricação, e suas não linearidades foram extraídas utilizando o mesmo método reportado anteriormente (IFM), com $\mathrm{V}_{\mathrm{A}}=50 \mathrm{mV}$ e $\mathrm{V}_{\mathrm{DS}}=750 \mathrm{mV}$.

\subsubsection{Corrente e Transcondutância dos Multifins}

Conforme relatado no capítulo 2, os dispositivos multifins são utilizados para maximizar a corrente drenada do conjunto, e, portanto, uma vez possuindo os dados desta arquitetura é interessante comprovar tal característica. Além disso, maiores valores de IDS resultam em maiores valores de transcondutância. A Figura 47 e a Figura 48 trazem estes dados.

Figura 47 - IDs em função de $V_{\text {GT }}$ para nanofios empilhados multifins com $\mathrm{W}_{\mathrm{FIN}}$ variados @ $\mathrm{V}_{\mathrm{DS}}=750 \mathrm{mV}$

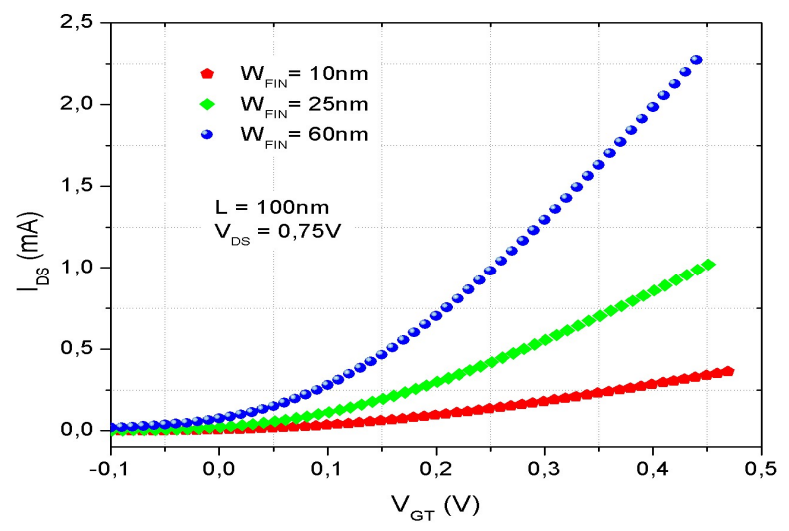

Fonte: Autor 
Figura 48 - $\mathrm{g}_{\mathrm{m}}$ em função de $\mathrm{V}_{\mathrm{GT}}$ para nanofios empilhados multifins com $\mathrm{W}_{\mathrm{FIN}}$ variados $@ \mathrm{~V}_{\mathrm{DS}}=750 \mathrm{mV}$

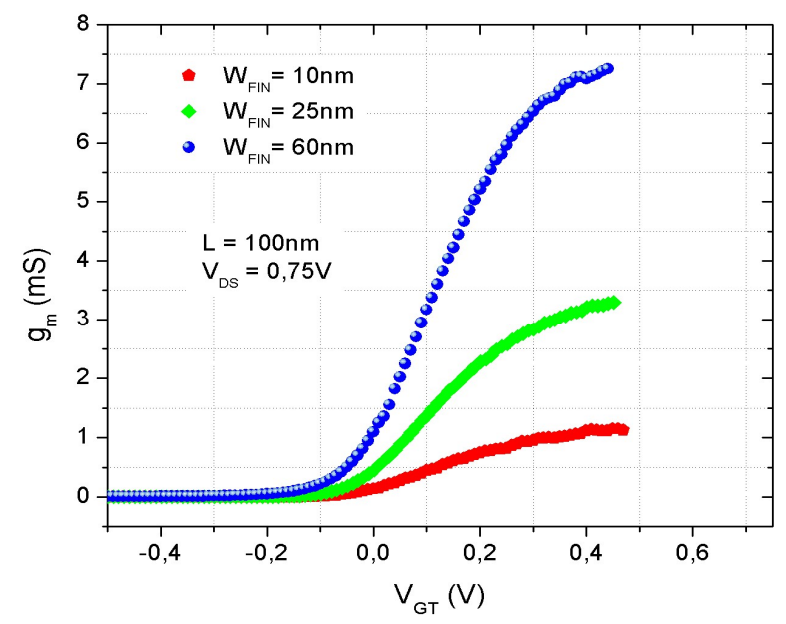

Fonte: Autor

É possível notar que, de fato, a magnitude da corrente de dreno e da transcondutância nesta condição é muito maior do que aquela vista na Figura 30 e na Figura 38, já que os gráfícos dos multifins possuem escala em $\mathrm{mA}$ e $\mathrm{mS}$, ao invés de $\mu \mathrm{A}$ e $\mu \mathrm{S}$. Estes dados foram utilizados nas próximas análises.

\subsubsection{Distorção Harmônica em função de VGT para os Nanofios Empilhados Multifins e $\operatorname{com} L$ variável}

As distorções harmônicas dos próximos gráficos foram extraídas pelo método da função integral com $\mathrm{V}_{\mathrm{A}}=50 \mathrm{mV}$ e nas mesmas condições de polarização que as figuras dos itens anteriores. Primeiramente, é mostrada a influência da largura na não linearidade dos dispositivos multifins com $\mathrm{L}=100 \mathrm{~nm}$, na Figura 49. 
Figura 49 - THD e HD3 em função de $\mathrm{V}_{\text {GT }}$ para $\mathrm{W}_{\text {FIN }}$ variável de multifins com L=100 nm@ $\mathrm{V}_{\mathrm{DS}}=750 \mathrm{mV}$
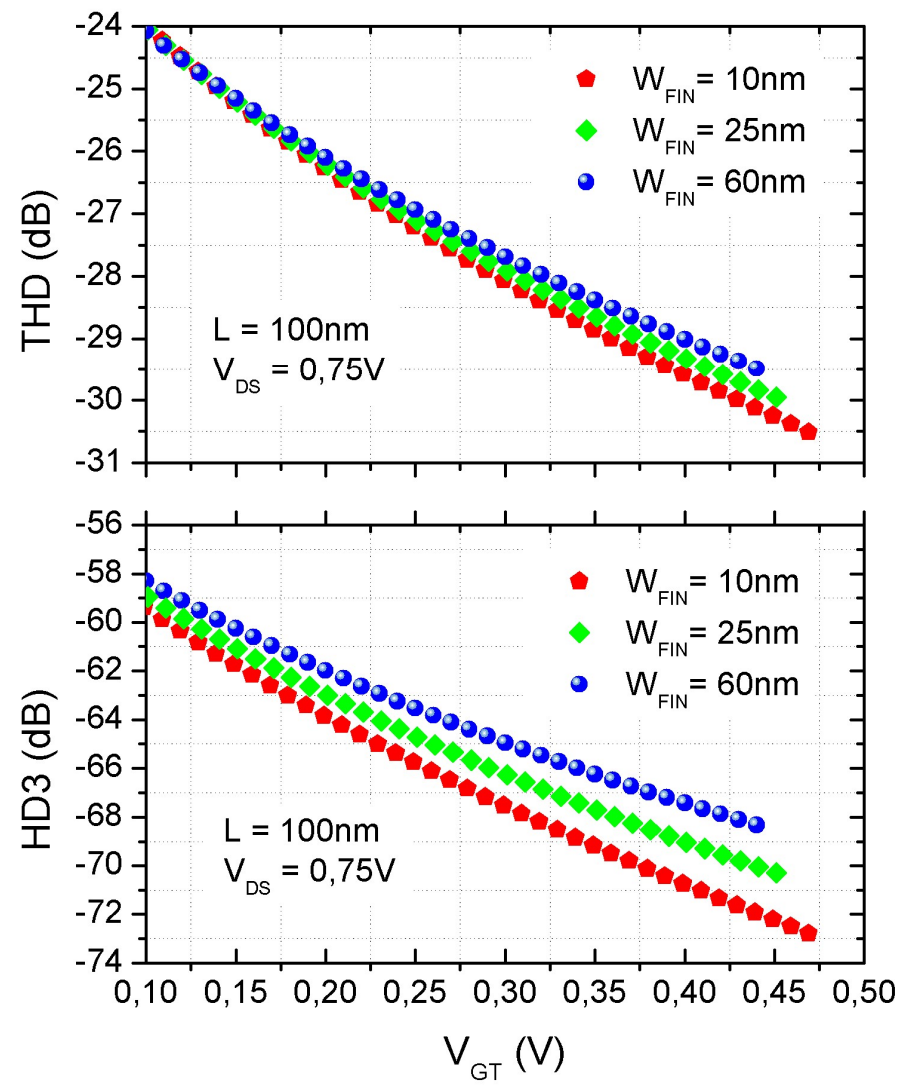

Fonte: Autor

Os resultados mostram que, tanto para THD quanto HD3, os transistores com maiores larguras de canal possuem maior distorção harmônica, o que concorda com os resultados apresentados na seção 5.2. Além disso, a trajetória da curva se manteve, com menores valores de distorção harmônica para maiores valores de $\mathrm{V}_{\mathrm{GT}}$. A Figura 50 mostra os resultados para o novo conjunto de dados com L variável. 
Figura 50 - THD e HD3 em função de $\mathrm{V}_{\mathrm{GT}}$ para L variável e $\mathrm{W}_{\mathrm{FIN}}=10 \mathrm{~nm} @ \mathrm{~V}_{\mathrm{DS}}=750 \mathrm{mV}$

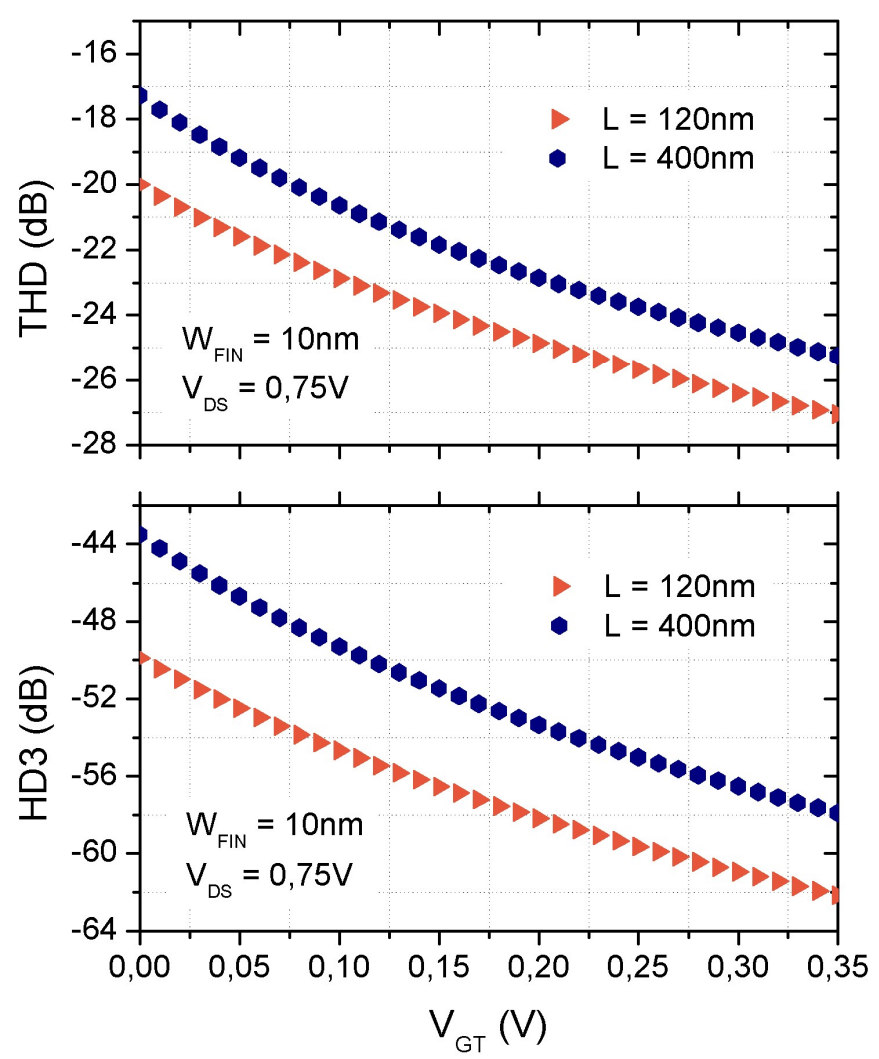

Fonte: Autor

O novo conjunto de dados reforça a relação entre o aumento de $\mathrm{L}$ e os maiores valores de distorção harmônica, o que também foi verificado na Figura 37 e justificado pelas equações (49) e (50). Os resultados também estão coerentes com os transistores de $\mathrm{L}=100 \mathrm{~nm}$ e L $=200$ $\mathrm{nm}$, já que a curva de $\mathrm{L}=120 \mathrm{~nm}$ possui valores intermediários e a de $\mathrm{L}=400 \mathrm{~nm}$ possui distorções superiores.

\subsubsection{Distorção Harmônica em função de $\mathrm{g}_{\mathrm{m}} / \mathbf{I}$ ds para os Nanofios Empilhados Multifins e} com L variável

Com o objetivo de demonstrar a similaridade de resultados de distorção em função de $\mathrm{g}_{\mathrm{m}} / \mathrm{I}_{\mathrm{DS}}$, novos gráficos foram gerados para os novos transistores, como pode ser visto na Figura 51. 


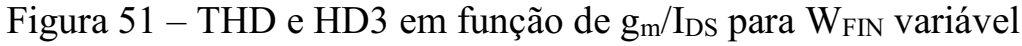
de multifins com $\mathrm{L}=100 \mathrm{~nm} @ \mathrm{~V}_{\mathrm{DS}}=750 \mathrm{mV}$
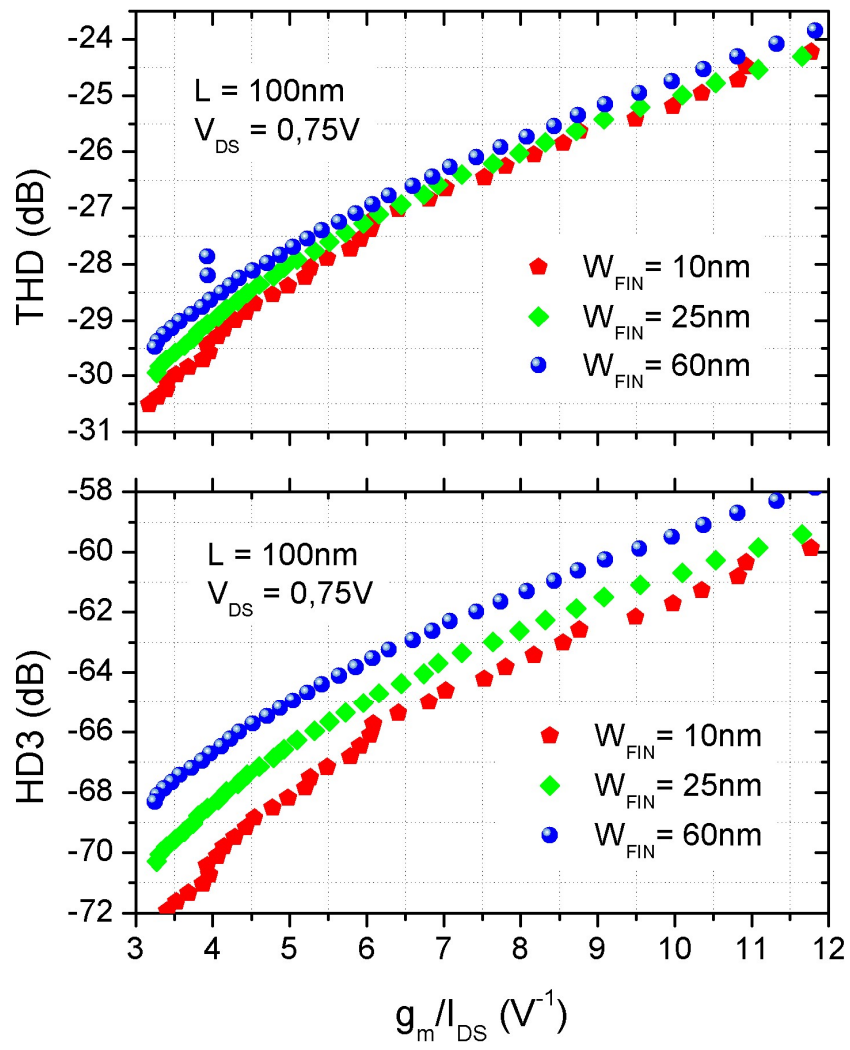

Fonte: Autor

Novamente, a análise converge para a relação entre o aumento da distorção harmônica para nanofios empilhados com maiores larguras de canal: $\mathrm{W}_{\mathrm{FIN}}=60 \mathrm{~nm}$ se mostrou o transistor com maior não linearidade em THD e HD3. A direcionalidade inversa em comparação ao gráfico de distorção em função de $\mathrm{V}_{\mathrm{GT}}$ também está alinhado com a Figura 40.

A Figura 41 trouxe valores muito próximos de distorção harmônica entre os nanofios empilhados de $\mathrm{L}=100 \mathrm{~nm}$ e $\mathrm{L}=200 \mathrm{~nm}$, o que eleva a relevância da análise para o novo conjunto de transistores, que pode ser observada na Figura 52. 
Figura 52 - THD e HD3 em função de $\mathrm{g}_{\mathrm{m}} / \mathrm{I}_{\mathrm{DS}}$ para L variável e $\mathrm{W}_{\mathrm{FIN}}=10 \mathrm{~nm} @ \mathrm{~V}_{\mathrm{DS}}=750 \mathrm{mV}$

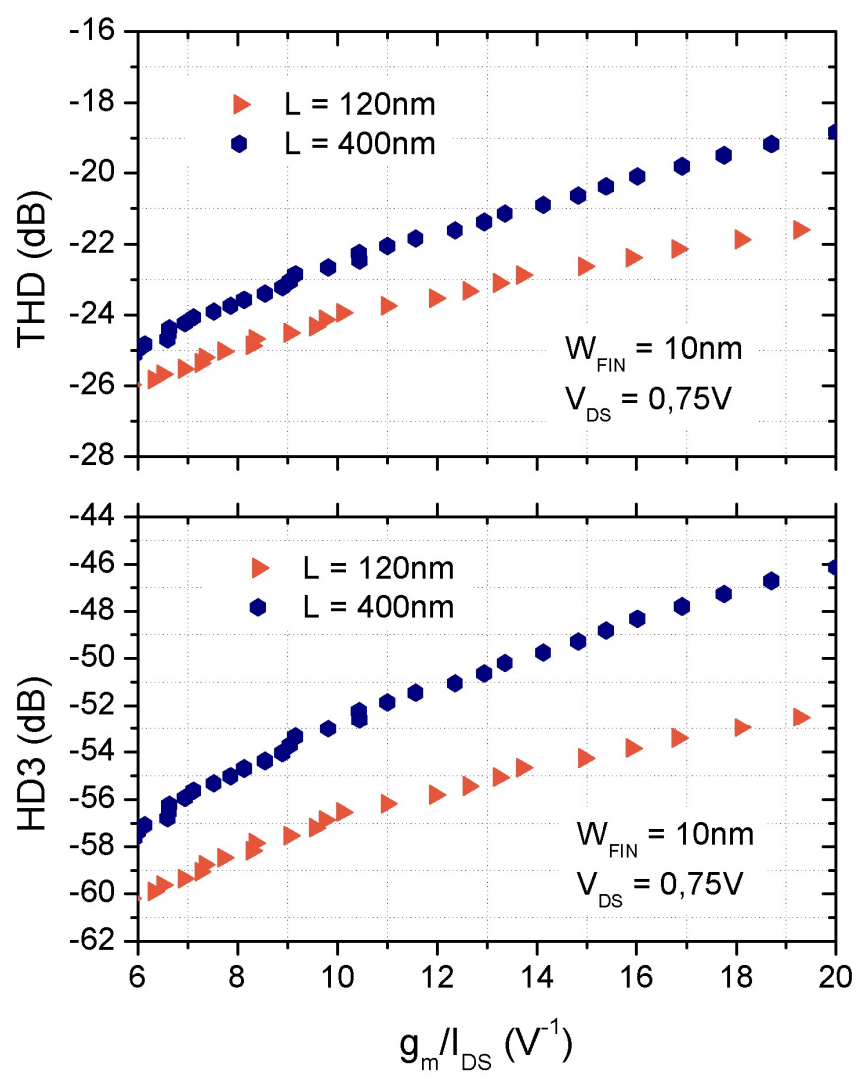

Fonte: Autor

Desta vez, foi possível separar as curvas com total clareza, demonstrando a maior distorção harmônica no transistor de $\mathrm{L}=400 \mathrm{~nm}$. Portanto, estas curvas permitem reforçar a relação entre comprimento de canal e não linearidade dos transistores nanofios empilhados.

\subsubsection{Distorção Harmônica dividida pelo Ganho em função de $\mathbf{g}_{\mathrm{m}} / \mathrm{IDS}$ para os Nanofios} Empilhados Multifins

A terceira estratégia empregada anteriormente consistiu em evidenciar THD e HD3 considerando seus diferentes ganhos. Primeiramente, foram extraídos os valores de Av para os multifins, vide Figura 53. 
Figura 53 - Av em função de $g_{m} / I_{D S}$ para $W_{\text {FIN }}$ variáveis com $\mathrm{L}=100 \mathrm{~nm} @ \mathrm{~V}_{\mathrm{GT}}=100 \mathrm{~nm}$

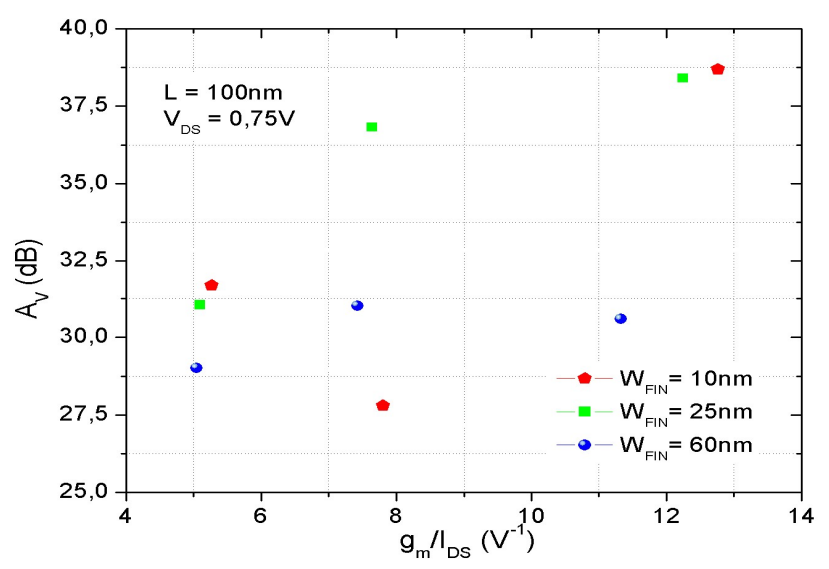

Fonte: Autor

De modo geral, os ganhos aumentaram com o incremento de $\mathrm{g}_{\mathrm{m}} / \mathrm{I}_{\mathrm{DS}}$, como era esperado. A exceção se deu principalmente no segundo ponto de $\mathrm{W}_{\mathrm{FIN}}=10 \mathrm{~nm}$, que se mostrou muito distante do ideal. Tal desvio foi atribuído a um problema pontual de medição. Apesar disto, o gráfico concorda com o que foi apresentado na Figura 42, uma vez que em ambas as figuras, os menores valores de $A_{V}$ foram obtidos para os transistores com maiores $\mathrm{W}_{\text {FIN }}$. Em posse destes resultados, gerou-se a curva de distorção dividia pelos respectivos ganhos, como pode ser observado na Figura 54.

Figura 54 - THD/Av e HD3/Av em função de $g_{m} / I_{D s}$ para $W_{\text {FIN }}$ variável com $\mathrm{L}=100 \mathrm{~nm} @ \mathrm{~V}_{\mathrm{DS}}=750 \mathrm{mV}$

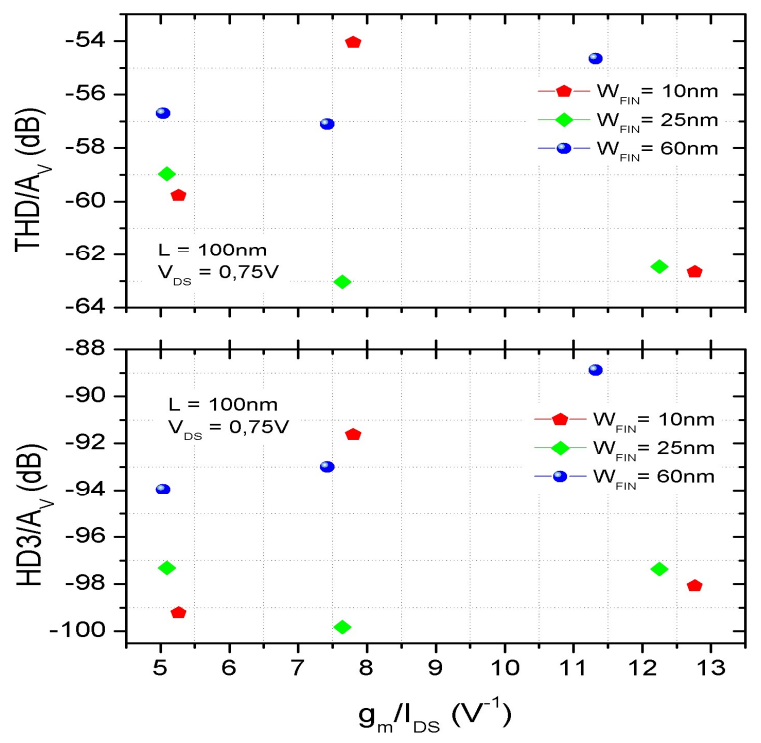

Fonte: Autor 
As novas curvas trazem mais uma confirmação: assim como na Figura 48, as maiores não linearidades ocorreram novamente em transistores com maiores larguras de canal. O desvio no segundo ponto do transistor de $\mathrm{W}_{\mathrm{FIN}}=10 \mathrm{~nm}$ foi gerado pela falha reportada no gráfico da Figura 53, porém todo o restante dos resultados está de acordo com as conclusões iniciais.

\subsubsection{Variação da transcondutância em função de $\mathbf{g}_{\mathrm{m}} / \mathrm{I}_{\mathrm{DS}}$ para os Multifins}

A última comparação possibilitará verificar se a relação entre $\partial g_{m} / \partial V_{G T}$ e as distorções harmônicas para os diferentes W Fin se mantém conforme a Figura 46. A partir da Figura 55, pode-se verificar que os transistores que possuem maior variação de $\partial g_{m} / \partial V_{G T}$ são os que possuem maior largura de canal, reforçando a relação de $W_{\text {FIN }}$ com a distorção harmônica, em mais uma concordância com a eq. (33).

Figura 55 - Resultados de $d g_{m} / d_{\text {GT }}$ em função de $g_{m} / I_{D S}$ para multifins com $\mathrm{W}_{\text {FIN }}$ variados

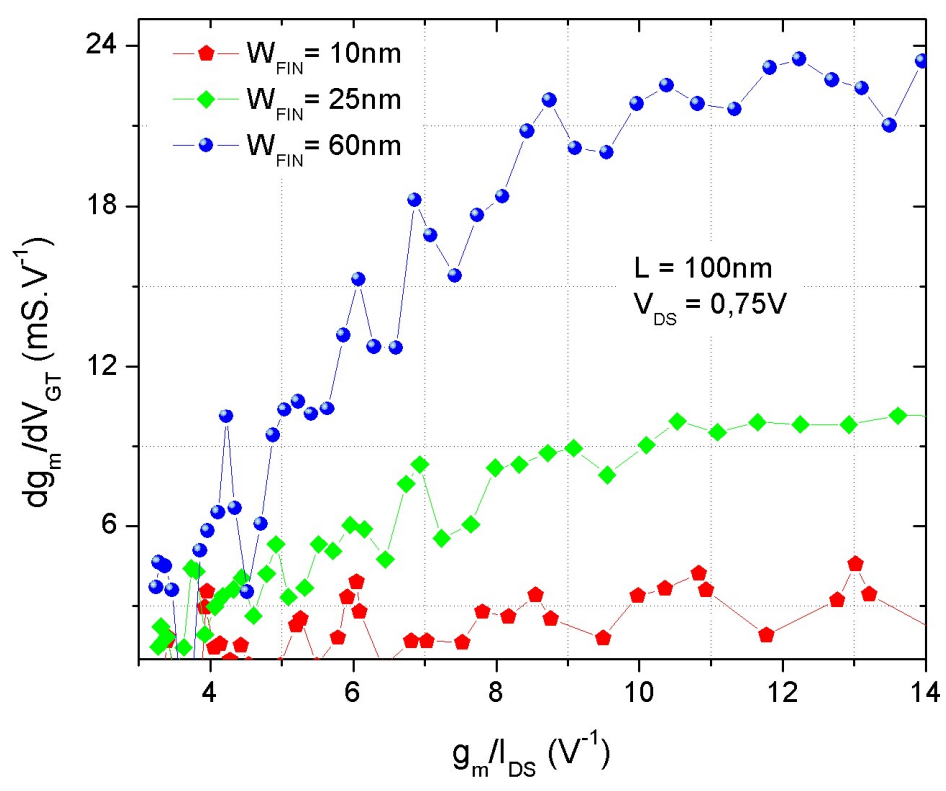

Fonte: Autor 


\section{CONCLUSÕES}

A pesquisa aqui retratada almejou elucidar as características analógicas referentes à distorção harmônica dos inovadores transistores nanofios empilhados, configurados como amplificadores operacionais de um único transistor. A partir dos resultados expostos, concluise que embora a arquitetura dos componentes seja diferente dos transistores SOI convencionais, suas dependências, equações matemáticas, comportamentos e relações físicas possuem grandes semelhanças entre si.

A natureza não linear dos transistores empilhados provoca distorções com grande preponderância e influência do segundo harmônico, sendo este, em média $30 \mathrm{~dB}$ maior do que a distorção proveniente do terceiro harmônico. Os gráficos demonstraram que existe uma relação entre a largura de canal dos dispositivos e a sua não linearidade, haja visto que em todas as análises os nanofios com maiores $\mathrm{W}_{\mathrm{FIN}}$ se apresentaram como os elementos de maior distorção harmônica, resultado este que está de acordo com os dados da literatura para os nanofios de único nível. Foi demonstrado matematicamente que o efeito é causado em decorrência da maior variação de transcondutância em função da sobretensão de porta existente para os nanofios empilhados com maiores valores de $\mathrm{W}_{\mathrm{FIN}}$. Além disso, dispositivos com maiores comprimentos de canal também apresentaram maiores distorções harmônicas - o que foi melhor evidenciado no novo conjunto de dados - cuja explicação matemática também foi apresentada.

Os resultados obtidos para os transistores nanofios empilhados multifins, além de concordarem com a relação previamente estabelecida para os componentes singulares entre maior distorção harmônica para dispositivos mais largos, se mostraram como uma excelente ferramenta de conclusão e comprovação, já que sua maior magnitude de corrente permitiu separar melhor as curvas e trazer maior confiabilidade aos resultados em todos os modos de análise.

Em virtude do grande número de efeitos acarretados a partir das distorções harmônicas, discutidos e explorados no capítulo 3, torna-se cada vez mais importante trazer à tona e inserir este tema em propostas de pesquisas científicas, sobretudo àquelas que tratam do comportamento analógico de novos dispositivos. $\mathrm{O}$ método da função integral declarado no capítulo 4 se mostrou uma ferramenta de suma importância neste processo, facilitando a aquisição e cálculo de dados, possibilitando assim, a evolução e desenvolvimento do estudo. 
Como sugestão para continuidade da investigação, pesquisas futuras podem utilizar modelos matemáticos e algoritmos computacionais para simular a integração dos nanofios empilhados em pequenos circuitos e/ou microssistemas, de forma a explorar a performance de diversos componentes em operação conjunta e dedicados a alguma aplicação específica, cujo desempenho seja prioritariamente baseado na distorção harmônica, como em amplificadores high-fidelity. 


\section{REFERÊNCIAS}

AGARWAL, T. What is a MOSFET : Working and Its Applications. ELPROCUS, 2020. Disponivel em: https://www.elprocus.com/mosfet-as-a-switch-circuit-diagram-free-circuits/. Acesso em: 15 set. 2020.

ALVES, C. R. Análise de descasamento nas características elétricas de SOI nMOSFET de canal gradual operando em saturação. 2017. 114 f. Dissertação (Mestrado em Engenharia Elétrica) - Centro Universitário FEI, São Bernardo do Campo. 2017. Disponível em: https://repositorio.fei.edu.br/bitstream/FEI/289/1/fulltext.pdf. Acesso em: 2 fev. 2021.

ARRIGALA, J.; WATSON, N. R. Power system harmonics. 2a . ed. Nova Zelândia: John Wiley \& Sons, 2003.

AZEVEDO, G. T. D.; ROMÃO, E. C.; MENEGATTI, C. R. Correção de distorções harmônicas em sistemas elétricos através de interferência destrutiva. Revista Brasileira de Ensino de Física, São Paulo, v. 41, n. 3, fev. 2019.

BALESTRA, F. et al. Double-gate silicon-on-insulator transistor with volume inversion: A new device with greatly enhanced performance. IEEE Electron Device Letters, set. 1987. 410-412. DOI: 10.1109/EDL.1987.26677. Disponível em:

https://ieeexplore.ieee.org/document/1487227. Acesso em: 22 mar. 2021.

BARRAUD, S. et al. Tunability of parasitic channel in gate-all-around stacked nanosheets. IEEE International Electron Devices Meeting (IEDM). São Francisco: IEEE. 2018. p. 21.3.1-21.3.4. DOI: 10.1109/IEDM.2018.8614507. Acesso em: 17 set. 2020.

BENFICA, A. T. O que são harmônicas em sistemas elétricos? Youtube, 2017. Disponivel em: https://www.youtube.com/watch?v=72gelIKLKiE. Acesso em: 22 out. 2020.

BRANT, T. Tri-Gate transistor. PCMag, 2016. Disponivel em:

https://www.pcmag.com/encyclopedia/term/tri-gate-transistor. Acesso em: 8 out. 2020.

BREWS, J. R. Subthreshold behaviour of uniformly and nonuniformly doped long-channel. IEEE Transactions on Electron Devices, 1979. 1282-1291. DOI: 10.1109/T-

ED.1979.19594. Disponível em: https://ieeexplore.ieee.org/document/1480172. Acesso em: 11 jan. 2021.

CAUGHEY, D. M.; THOMAS, R. E. Carrier mobilities in silicon empirically related to doping and field. Proceedings of the IEEE, v. 55, no. 12, p. 2192-2193, dez. 1967. DOI: 10.1109/PROC.1967.6123. Disponível em: https://ieeexplore.ieee.org/document/1448053. Acesso em: 23 jan. 2021.

CERDEIRA, A. et al. Integral function method for determination of non-linear harmonic distortion. Solid-State Electronics, 2004. 2225-2234. DOI:

https://doi.org/10.1016/j.sse.2004.06.001. Disponível em: https://www.sciencedirect.com/science/article/abs/pii/S0038110104002321. Acesso em: 14 out. 2020 . 
CEUSTER, D. D.; FLANDRE, E. Kink-like effect in long n-channel twin-gate fully depleted SOI MOSFETs. Electronics Letters, p. 1456-1458, 1994. DOI:

https://doi.org/10.1049/el:19940992. Disponível em:

https://dial.uclouvain.be/pr/boreal/object/boreal:48729. Acesso em: 22 jan. 2021.

CHENG, Y. H. Comparison of MOSFET threshold voltage extraction methods with temperature variation. IEEE 32nd International Conference on Microelectronic Test Structures (ICMTS). Kitakyushu: IEEE. 2019. p. 126-131. DOI:

10.1109/ICMTS.2019.8730978. Disponível em:

https://www.researchgate.net/publication/344744956_Comparison_of_MOSFET_Threshold_ Voltage_Extraction_Methods_with_Temperature_Variation. Acesso em: 17 jan. 2021.

COELHO, J. M. Não podemos prever o futuro, mas podemos criá-lo. Valor Globo. 2018. Disponível em: https:/valor.globo.com/financas/coluna/nao-podemos-prever-o-futuro-maspodemos-cria-lo.ghtml. Acesso em: 26 maio 2021.

COLINGE, J. P. Problems and issues in SOI CMOS technology. IEEE International SOI Conference Proceedings. Vail Valley: IEEE. 1991. p. 126-127. DOI:

10.1109/SOI.1991.162889. Disponível em: https://ieeexplore.ieee.org/document/162889. Acesso em: 24 jan. 2021.

COLINGE, J. P. Silicon-on-insulator technology: Materials do VLSI. $3^{\text {a }}$. ed. Nova York: Kluwer Academic Publishers, 2004.

COLINGE, J. P. et al. Silicon-on-insulator "gate-all-around device". Technical Digest of IEDM. São Francisco: IEEE. 1990. p. 595-598. DOI: 10.1109/IEDM.1990.237128.

Disponível em: https://ieeexplore.ieee.org/document/237128. Acesso em: 14 jan. 2021.

COLINGE, J. P. et al. Silicon-on-insulator "gate-all-around" MOS device. IEEE SOS/SOI Technology Conference. Key West: IEEE. 1990. p. 137-138. DOI:

10.1109/SOSSOI.1990.145749. Disponível em: https://ieeexplore.ieee.org/document/145749. Acesso em: 14 dez. 2020.

COLINGE, J. P. et al. Low-temperature electron mobility in trigate SOI MOSFETs. IEEE Electron Device Letters, v. 27, no 2., p. 120-122, Fev. 2006. DOI:

10.1109/LED.2005.862691. Disponível em: https://ieeexplore.ieee.org/document/1580601. Acesso em: 2 fev. 2021.

CONDE, A. O. et al. Revisiting MOSFET threshold voltage extraction methods.

Microelectronics Reliability, 13 nov. 2013. 90-104. DOI:

https://doi.org/10.1016/j.microrel.2012.09.015. Disponível em:

https://www.sciencedirect.com/science/article/abs/pii/S0026271412004684. Acesso em: 3 fev. 2021.

DIXIT, A. et al. Analysis of the parasitic S/D resistance in multiple-gate FETs. IEEE Transactions on Electron Devices, v. 52, n. 6, p. 1132-1140, jun. 2005. DOI:

10.1109/TED.2005.848098. Disponível em: https://ieeexplore.ieee.org/document/1433106. Acesso em: 12 jan. 2021. 
DORIA, R. T. Operação analógica de transistores de mútiplas portas em função da temperatura. 2010. 198 f. Tese (Doutorado em Engenharia Elétrica) - Escola Politécnica da Universidade de São Paulo. São Paulo. 2010. Disponível em:

https://teses.usp.br/teses/disponiveis/3/3140/tde-10012011-135500/pt-br.php. Acesso em: 7 out. 2020 .

DORIA, R. T. et al. Harmonic distortion of unstrained and strained FinFETs operating in saturation. IEEE Transactions on Electron Devices, v. 57, p. 3303-3311, dez. 2010. ISSN DOI: 10.1109/TED.2010.2079936. Disponível em:

https://ieeexplore.ieee.org/document/5613164. Acesso em: 12 out. 2020.

DOYLE, B. S. . E. A. High performance fully-depleted tri-gate CMOS transistors. IEEE Electron Device Letters, p. 263-265, 2003. DOI: 10.1109/LED.2003.810888. Disponível em: https://ieeexplore.ieee.org/document/1206858. Acesso em: 23 out. 2020.

DUARTE, J. P. et al. A universal core model for multiple-gate field-effect transistors. Part II: drain current model. IEEE Transactions on Electron Devices, v. 60, n. 2, p. 848-855, Fev. 2013. DOI: 10.1109/TED.2012.2233863. Disponível em:

https://ieeexplore.ieee.org/document/6403538. Acesso em: 14 jan. 2021.

DUPRE, C. 3D stacked nanowires MOSFET with optional independent gate operation ( $\phi F E T$ ): fabrication and transport properties. 2008. $204 \mathrm{f}$. Tese (Doutorado) - Instituto Politécnico de Grenoble. Grenoble. 2008.

DUPRÉ, C. et al. 15nm-diameter 3D stacked nanowires with independent gates operation: $\Phi F E T$. IEEE International Electron Devices Meeting. San Francisco, CA: IEEE. 2008. p. 1-4. DOI: 10.1109/IEDM.2008.4796805. Disponível em: https://ieeexplore.ieee.org/document/4796805. Acesso em: 12 nov. 2020.

ERNST, T. et al. Novel 3D integration process for highly scalable Nano-Beam stackedchannels GAA (NBG) FinFETs with HfO2/TiN gate stack. 2006 International Electron Devices Meeting. San Francisco: IEEE. 2006. DOI: 10.1109/IEDM.2006.346955. Disponível em: https://ieeexplore.ieee.org/document/4154390. Acesso em: 19 dez. 2020.

ESPINOZA, H. R. Efecto skin en conductores. Solarpraxis, 2012. Disponivel em: http://solarpraxis.blogspot.com/2012/02/efecto-skin-en-conductores.html. Acesso em: 8 nov. 2020 .

FECHNERAND, P. S.; VOGT, E. E. Oxidation induced stress effects on hole mobility as function of transistor geometry in a $0.15 / \mathrm{spl} \mathrm{mu/m}$ dual gate oxide CMOS SOI process. 2005 IEEE International SOI Conference Proceedings. IEEE. 2005. p. 163-165. DOI: 10.1109/SOI.2005.1563575. Disponível em: https://ieeexplore.ieee.org/document/1563575. Acesso em: 7 abr. 2021.

FLANDRE, D. et al. Design of thin-film fully depleted SOI CMOS analog circuits significantly outperforming bulk implementations. Proceedings 1994 IEEE International SOI Conference. Nantucket: IEEE. 1994. p. 99-100. DOI: 10.1109/SOI.1994.514265. Disponível em: https://ieeexplore.ieee.org/document/514265. Acesso em: 29 set. 2020. 
GAO, M. et al. Twin MOSFET structure for supression of kink and parasitic bipolar effects in SOI MOSFETs at room and liquid helium temperatures. Solid-State Electron, p. 505-512, 1992. DOI: https://doi.org/10.1016/0038-1101(92)90112-P. Disponível em:

https://www.sciencedirect.com/science/article/abs/pii/003811019290112P. Acesso em: 1 out. 2020 .

GHIBAUDO, G. New method for the extraction of MOSFET parameters. Electronics Letters, Maio 1988. 543-545. DOI: 10.1049/el:19880369. Disponível em:

https://www.researchgate.net/publication/3365397_New_method_for_the_extraction_of_MO SFET_parameters. Acesso em: 4 out. 2020.

GROENEWOLD, G.; LUBBERS, W. J. Systematic distortion analysis for MOSFET integrators with use of a new MOSFET model. IEEE Trans. Circuits Syst. II Analog Digit. Signal Process, p. 569-580, 1994. DOI: 10.1109/82.326583. Disponível em: https://ieeexplore.ieee.org/document/326583. Acesso em: 26 out. 2020.

GUPTA, D. et al. Digital output data links from superconductor integrated circuits. IEEE Transactions on Applied Superconductivity, vol. 29, no. 5, p. 1-8, 2019. DOI:

10.1109/TASC.2019.2910469. Disponível em: https://ieeexplore.ieee.org/document/8686133. Acesso em: 17 ago. 2020.

GUSBERTI, G. F. Distorção harmônica e não linearidade! Youtube, 2018. Disponivel em: https://www.youtube.com/watch?v=OdYCdb7qFOo. Acesso em: 23 out. 2020.

JOHANSSON, H.; WANHAMMAR, L. High-speed recursive digital filters based on the frequency-response masking approach. IEEE Transactions on Circuits and Systems II: Analog and Digital Signal Processing, vol. 47, no. 1, p. 48-61, 2000. DOI: 10.1109/82.818894. Disponível em: https://ieeexplore.ieee.org/document/818894. Acesso em: 19 ago. 2020.

JURCZAK, M.; JAKUBOWSKI, A. Improved description of GAA (gate-all-around) MOSFET I-V characteristics. ESSDERC '96: Proceedings of the 26th European Solid State Device Research Conference. Bolonha: IEEE. 1996. p. 283-286. Disponível em: https://ieeexplore.ieee.org/document/5436368. Acesso em: 11 out. 2020.

KRISHNAN, S. et al. Floating-body kinks and dynamic effects in fully depleted SOI MOSFE Ts. Proceedings 1995 IEEE International SOI Conference. Tucson: IEEE. 1995. p. 10-11. DOI: 10.1109/SOI.1995.526435. Disponível em: https://ieeexplore.ieee.org/document/526435. Acesso em: 14 set. 2020.

LU, N.; LEE, S.; WACHNIK, R. A. Symmetry breaking in the drain current of multifinger transistors. 2015 IEEE Custom Integrated Circuits Conference (CICC). San Jose: IEEE. 2015. DOI: 10.1109/CICC.2015.7338408. Disponível em: https://ieeexplore.ieee.org/document/7338408. Acesso em: 29 out. 2020.

MANASEVIT, H. M.; SIMPSON, W. I. Single-crystal silicon on a sapphire substrate. Journal of Applied Physics, v. 35, p. 1349-1351, 1964. DOI: https://doi.org/10.1063/1.1713618. Disponível em: https://aip.scitation.org/doi/10.1063/1.1713618. Acesso em: 18 mar. 2020. 
MARINIELLO, G. et al. Evaluation of analog characteristics of n-type vertically stacked nanowires. Caen: EUROSOI-ULIS. 2020. DOI: 10.1109/EUROSOI-

ULIS49407.2020.9365636. Disponível: https://ieeexplore.ieee.org/document/9365636.

Acesso em: 28 nov. 2020.

MARTINS, D. C.; BARBI, I. Eletrônica de potência: Introdução ao estudo de conversores CC-CA. Florianópolis: [s.n.], 2008.

MEHTA, N.; HUIJSING, J. H.; STOJANOVIC, V. A 1-mW class-AB amplifier with -101 dB THD+N for high-fidelity 16 omega headphones in 65-nm CMOS. IEEE Journal of SolidState Circuits, Abr. 2019. 948-958. DOI: 10.1109/JSSC.2018.2886320. Disponível em: https://ieeexplore.ieee.org/document/8600336. Acesso em: 14 ago. 2020.

MERRIKHI, J.; MOGHANI, J. S.; FALLAH, E. Modeling flux skin effect on the harmonic currents of TCR. Power Electronics Systems and Applications, 2006. 113-114. DOI: 10.1109/PESA.2006.343081. Disponível em: https://ieeexplore.ieee.org/document/4147794. Acesso em: 3 nov. 2020.

MORAES, C. P. A.; SOUZA, M. D. Estudo comparativo de métodos de extração de tensão de limiar em transistores SOI nMOSFET. Centro Universitário FEI. São Bernardo do Campo. 2014. Disponível em:

https:/fei.edu.br/70anos/simposio/trabalhos2014/eletrica/caroline_moraes_michelly_de_souz a.pdf. Acesso em: $11 \mathrm{dez} .2020$.

NEMER, J. P. Desenvolvimento de nanofios transistores em substratos SOI com espessuras nanométricas. 2017. $133 \mathrm{f}$. Tese (Doutorado em Engenharia Elétrica) - Centro Universitário FEI. São Bernardo do Campo, 2017. DOI: 10.31414/EE.2017.T.129320.

Disponível em: https://repositorio.fei.edu.br/handle/FEI/292. Acesso em: 19 nov. 2020.

NORRIS, C. B.; GIBBONS, J. F. Measurement of high-field carrier drift velocities in silicon by a time-of-flight technique. IEEE Transactions on Electron Devices, v. 14, no. 1, p. 3843, Jan. 1967. DOI: 10.1109/T-ED.1967.15893. Disponível em:

https://ieeexplore.ieee.org/document/1474616. Acesso em: 12 set. 2020.

NOVELIN, I. T. Comportamento harmônico de inversores de frequência. 2017. 73 f. TCC (Graduação em Engenharia Elétrica) - Universidade Tecnológica Federal do Paraná. Curitiba. 2017. Disponível em: https://repositorio.utfpr.edu.br/jspui/bitstream/1/10046/1/CT_COELE_2017_2_15.pdf. Acesso em: 2 nov. 2020.

ORTMEYER, T. H.; CHAKRAVARTHI, K. R. The effects of power system harmonics on power system equipment and loads. IEEE Trans. on Power Apparatus and Systems, 1985. 2555-2563. DOI: 10.1109/MPER.1985.5526463. Disponível em:

https://ieeexplore.ieee.org/document/5526463. Acesso em: 2 nov. 2020.

PARTRIDGE, S. L. Silicon-on-insulator technology. IEE Proceedings E - Computers and Digital Techniques, 1986. 106-116. DOI: 10.1049/ip-i-1.1986.0017. Disponível em: https://digital-library.theiet.org/content/journals/10.1049/ip-i1.1986.0017; jsessionid=a6fkck9aaj2m.x-iet-live-01. Acesso em: 27 ago. 2020. 
PAVANELLO, M. A. Projeto, fabricação e caracterização elétrica de uma nova estrutura para o SOI MOSFET. 2020. 138 f. Tese (Doutorado em Engenharia Elétrica) - Escola Politécnica da USP. São Paulo. 2000.

PAVANELlO, M. A.; MARTINO, J. A.; FLANDRE, D. Analog circuit design using gradedchannel SOI nMOSFETs. Symposium on Integrated Circuits and Systems Design, Pirenopolis, 2001. p. 130-135. DOI: 10.1109/SBCCI.2001.953015. Disponível em: https://ieeexplore.ieee.org/document/953015. Acesso em: 14 dez. 2020.

PAZ, B. C. et al. Non-linearity analysis of triple gate SOI nanowires MOSFETS. 31st Symposium on Microelectronics Technology and Devices (SBMicro), Belo Horizonte, 2016. DOI: 10.1109/SBMicro.2016.7731355. Disponível em: https://ieeexplore.ieee.org/document/7731355. Acesso em: 8 dez. 2020.

PIDIN, S. et al. Modeling of impact ionization current for LDD SOI MOSFETs. ESSDERC '95: Proceedings of the 25th European Solid State Device Research Conference. Haia: IEEE. 1995. p. 393-396. Disponível em: https://ieeexplore.ieee.org/document/5435912. Acesso em: 11 dez. 2020.

RAI, S.; CHAUDHURI, M. Improving CPU performance through dynamic GPU access throttling in CPU-GPU heterogeneous processors. IEEE International Parallel and Distributed Processing Symposium Workshops (IPDPSW). Lake Buena Vista, FL: IEEE. 2017. p. 18-29. DOI: 10.1109/IPDPSW.2017.37. Disponível em: https://ieeexplore.ieee.org/document/7965022. Acesso em: 29 ago. 2020.

RICCO, B.; VERSARI, R.; ESSENI, D. A novel method to characterize parasitic capacitances in MOSFETs. IEEE Electron Device Letters, nov. 1995. 485-487. DOI: 10.1109/55.468275. Disponível em: https://ieeexplore.ieee.org/document/468275. Acesso em: 4 set. 2020.

ROSÉN, L.; SAMIMI, S. Small electronic load. Design and analysis of a small electronic load for testing on-board DC/DC converters. Universidade de Tecnologia de Chalmers. Goteborg. 2012. Disponível em: https://www.semanticscholar.org/paper/Small-electronicload.-Design-and-analysis-of-a-for-RosenSamimi/5806bced3529cb6f20f29722aae621c3192722f7. Acesso em: 15 out. 2020.

SAH, C.-T. et al. Effect of zinc impurity on silicon solar-cell efficiency. IEEE Transactions on Electron Devices, v. 28, no. 3, p. 304-313, mar. 1981. DOI: 10.1109/T-ED.1981.20333. Disponível em: https://ieeexplore.ieee.org/document/1481485. Acesso em: 20 mar. 2020.

SANTINO, R. Conheça os 3 supercomputadores brasileiros entre os mais potentes do mundo. Olhar Digital, 14 fev. 2020. Disponivel em: https://olhardigital.com.br/noticia/conheca-os-3supercomputadores-brasileiros-entre-os-mais-potentes-do-mundo/96844. Acesso em: 12 out. 2020 .

SANTOS, C. D. G. D. Estudo da mobilidade em dispositivos SOI planares e de múltiplas portas. 2010. 208 f. Tese (Doutorado em Engenharia) - Escola Politécnica da USP. São Paulo. 2010. Disponível em: https://www.teses.usp.br/teses/disponiveis/3/3140/tde10012011-093657/pt-br.php. Acesso em: 15 fev. 2020. 
SANTOS, H. L. Influência das distorções harmônicas na proteção de bancos de capacitores. 2010. 128 f. Dissertação (Mestrado em Engenharia Elétrica) - UFMG. Minas Gerais. 2010.

SEDRA, S.; SMITH, K. Microeletrônica 4a Edição. São Paulo: Pearson Makron Books, 2005.

SILVEIRA, C. B. O que faz o inversor de frequência e como especificar? Citisystems, 2019. Disponivel em: https://www.citisystems.com.br/inversor-de-frequencia/. Acesso em: 2 nov. 2020.

SILVEIRA, F.; FLANDRE, D.; JESPERS, P. G. A. A gm/Id based methodology for the design of CMOS analog circuits and its application to the synthesis of a silicon-on-insulator micropower OTA. IEEE Journal of Solid-State Circuits, vol. 31, set. 1996. 1314-1319. DOI: 10.1109/4.535416. Disponível em: https://ieeexplore.ieee.org/document/535416. Acesso em: 12 abr. 2021.

SONG, Y.; LUO, J.; LI, X. Vertically stacked individually tunable nanowire field effect transistor for low power operation with ultrahigh radio frequency linearity. Applied Physics Letters 101, ago. 2012. DOI: 10.1063/1.4747448. Disponível em:

https://www.researchgate.net/publication/257952798_Vertically_stacked_individually_tunabl e_nanowire_field_effect_transistors_for_low_power_operation_with_ultrahigh_radio_freque ncy_linearity. Acesso em: 14 nov. 2020.

SZE, S. M.; NG, K. K. Physics of semiconductor devices. 2a. ed. [S.1.]: J. Wiley \& Sons, 1981.

WOUTERS, D. J.; COLINGE, J. P.; MAES, H. E. Subthreshold current in thick and thinfilm SOI MOSFET transistors. IEEE SOS/SOI Technology Conference. Stateline, NV, USA: IEEE. 1989. DOI: 10.1109/SOI.1989.69746. Disponível em:

https://ieeexplore.ieee.org/document/69746. Acesso em: 23 out. 2020.

ZHAO, Y. et al. Comprehensive understanding of surface roughness limited mobility in unstrained- and strained-Si MOSFETs by novel characterization scheme of $\mathrm{Si} / \mathrm{SiO} 2$ interface roughness. 2009 Symposium on VLSI Technology. Honolulu, HI: IEEE. 2009. p. 18-19. Disponível em: https://ieeexplore.ieee.org/document/5200618. Acesso em: 14 abr. 2021. 
ANEXO A - FLUXOGRAMA DO PROCESSO DO MÉTODO DA FUNÇÃO INTEGRAL 


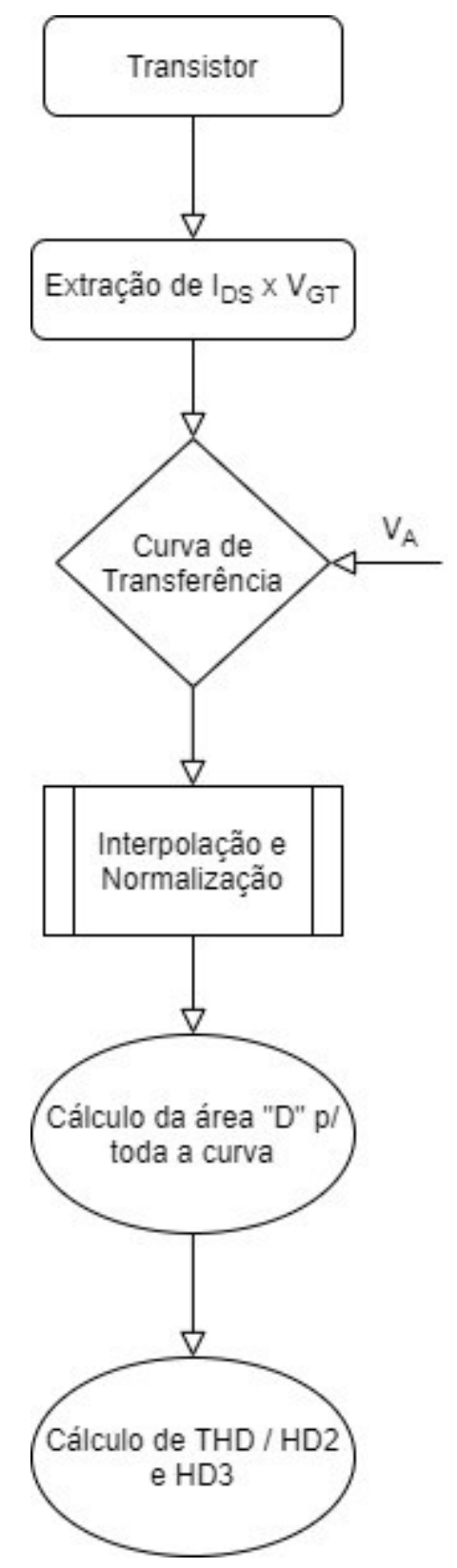




\section{ANEXO B - ARTIGO CIENTÍFICO PUBLICADO NA JOURNAL OF INTEGRATED CIRCUITS AND SYSTEMS (JICS)}




\title{
Performance and Analysis of n-Type Vertically Stacked Nanowires Regarding Harmonic Distortion
}

\author{
Cesar Augusto Belchior de Carvalho ${ }^{1}$, Genaro Mariniello ${ }^{1}$, Bruna Cardoso Paz ${ }^{2}$, Sylvain Barraud ${ }^{2}$, \\ Maud Vinet ${ }^{2}$, Olivier Faynot ${ }^{2}$, and Marcelo Antonio Pavanello ${ }^{1}$ \\ ${ }^{1}$ Department of Electrical Engineering, Centro Universitário FEI, São Bernardo do Campo, Brazil \\ ${ }^{2}$ CEA-LETI, Minatec Campus, and Univ. Grenoble Alpes, 38054 Grenoble, France \\ e-mail: ccarvalho@fei.edu.br
}

\begin{abstract}
This paper studies the harmonic distortion (or non-linearity) of vertically stacked SOI nanowires with different fin widths and channel lengths. The total harmonic distortion and third order harmonic distortion are used as figures of merit in this work. The harmonic distortion analysis is performed taking in consideration the differences between transistor's intrinsic voltage gain and transconductance over drain current ratio.
\end{abstract}

Index Terms-stacked nanowires; harmonic distortion; MOSFET; SOI transistor

\section{INTRODUCTION}

The continuous advance of Integrated Circuits (IC) allowing an increased number of functions per chip, triggered the search for solutions that can provide both, improved gate control to overcome the short-channel effects as well as larger drain current without increasing the consumed silicon area [1]. One recent alternative being explored is the use of vertically stacked nanowires. It consists of multiple 3D stacked nanosheet levels of MOSFETs sharing the same gate, source and drain electrodes, behaving as one single transistor. Depending on the technology, the lower level has a trigate nanowire architecture and the levels above have a Gate-All-Around architecture [2].

Despite the continuous engagement in research of suitable components for digital applications, it's crucial that analog characteristics of newer devices are studied and optimized as well, since mixed analog-digital circuits comprise a farreaching share of global electronic market. This fact is easily noticed by observing the amount of new products arriving and being consumed every day, which have a lot of functions that requires a decent digital/analog converter, such as mobile phones and computers with excellent cam recorders and sound speakers with better audio reproduction [3].

As the output characteristics of MOSFETs are very nonlinear, when an input sinusoidal signal of a certain frequency is applied, the output signal will have the fundamental frequency and several high-order harmonics that are undesired. The amount of these undesired harmonics represents the harmonic distortion. The harmonic distortion (or non-linearity) is then amongst the analog figures of merit of a device.

The harmonic distortion is accounted by looking at the total harmonic distortion (THD), that refers to the overall amount of distortion added by the device, and the third order harmonic distortion (HD3), which is the first odd harmonic in the output signal.
To correlate specifically the non-linearity from MOSFETS and a day-to-day application, it is possible to stablish a relation between a component's total harmonic distortion and high-fidelity (hi-fi) audio streaming. As seen in [3], the total harmonic distortion of a class-AB amplifier, for example, is a common measure to indicate the sound reproduction quality of our media players. In addition, signal transmission on radio frequency circuits and applications require flawless performance of linearity on its electronic systems. Studies validating previously mentioned data can be found in [4].

This work aims to explore the harmonic distortion of twolevel vertically stacked nanowires operating as single transistor amplifiers. Some of their basic electrical characteristics were presented in ref. [2]. However, to the best of our knowledge, the stacked nanowire harmonic distortion phenomenon has not been presented yet.

\section{CHARACTERISTICS OF THE MEASURED DEVICES}

The devices used in this work are 3D stacked nanosheets with two levels made in Silicon-On-Insulator substrate: the lower level has triple gate SOI MOSFET architecture and the upper level is a Gate-All-Around (GAA) Si transistor. They were manufactured by CEA-LETI, with both Si channels of $9 \mathrm{~nm}$ thickness $\left(\mathrm{H}_{\mathrm{FIN}}\right)$. The gate stack is composed of a $2 \mathrm{~nm}$ oxide $\left(\mathrm{HfO}_{2}\right)$ and a TiN and Tungsten metal [5].

Fig. 1 presents a TEM view of 2 stacked nanowires with different fin width $\left(\mathrm{W}_{\mathrm{FIN}}\right)$ [5]. The total device channel width of stacked nanowire is a sum of widths from two levels of the device.

$\mathrm{W}_{(\mathrm{STACKED})}=\mathrm{W}_{(\mathrm{TRIGATE})}+\mathrm{W}_{(\mathrm{GAA})}=4 \mathrm{H}_{\mathrm{FIN}}+3 \mathrm{~W}_{\mathrm{FIN}}$

Regarding non-linearity analysis, n-type transistors with variable $W_{\text {FIN }}$ of $10 \mathrm{~nm}, 20 \mathrm{~nm}, 40 \mathrm{~nm}$ and a channel length of $\mathrm{L}=100 \mathrm{~nm}$, and a variable channel length of $100 \mathrm{~nm}$ and $200 \mathrm{~nm}$ with a constant $\mathrm{W}_{\text {FIN }}$ equal to $10 \mathrm{~nm}$ were used.

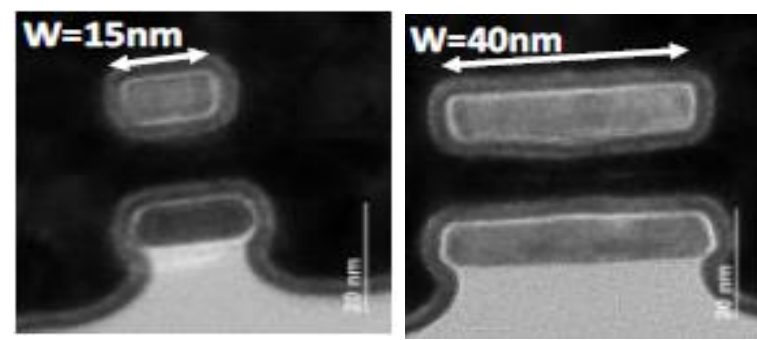

Fig.1 TEM image of stacked nanowires with different width [5] 
The extraction of harmonic distortion can be performed using $\mathrm{AC}$ measurements; however, these techniques are complex and require high demand on signal processing since they lead to a noisy output signal. The method itself adds non-linearities inherent of the extraction and signals of current are in $\mu \mathrm{A}$ magnitude [2].

On the other hand, the Integral Function Method (IFM) [6] allows the extraction of device's THD, HD3 and other distortion components using only DC curves of gate voltage and drain current. For devices operating as single transistor amplifiers, as in this work, the method assumes that an input sinusoidal signal with amplitude $\left(\mathrm{V}_{\mathrm{A}}\right)$ is applied together with DC gate voltage and the linearity is accounted in the drain current.

\section{RESULTS}

The measured drain current $\left(\mathrm{I}_{\mathrm{DS}}\right)$ as function of gate voltage overdrive $\left(\mathrm{V}_{\mathrm{GT}}=\mathrm{V}_{\mathrm{GS}}-\mathrm{V}_{\mathrm{TH}}, \mathrm{V}_{\mathrm{GS}}\right.$ being the gate voltage and $\mathrm{V}_{\mathrm{TH}}$ the threshold voltage), extracted with a drain voltage $\left(\mathrm{V}_{\mathrm{DS}}\right)$ of $750 \mathrm{mV}$, are presented in Fig. 2. It is possible to see the increase of $\mathrm{I}_{\mathrm{DS}}$ in larger $\mathrm{W}_{\mathrm{FIN}}$ and smaller channel length transistors, as expected. The maximum values for $\mathrm{V}_{\mathrm{GT}}$ were fixed on $0.33 \mathrm{~V}$ to preserve transistors gate oxide integrity.
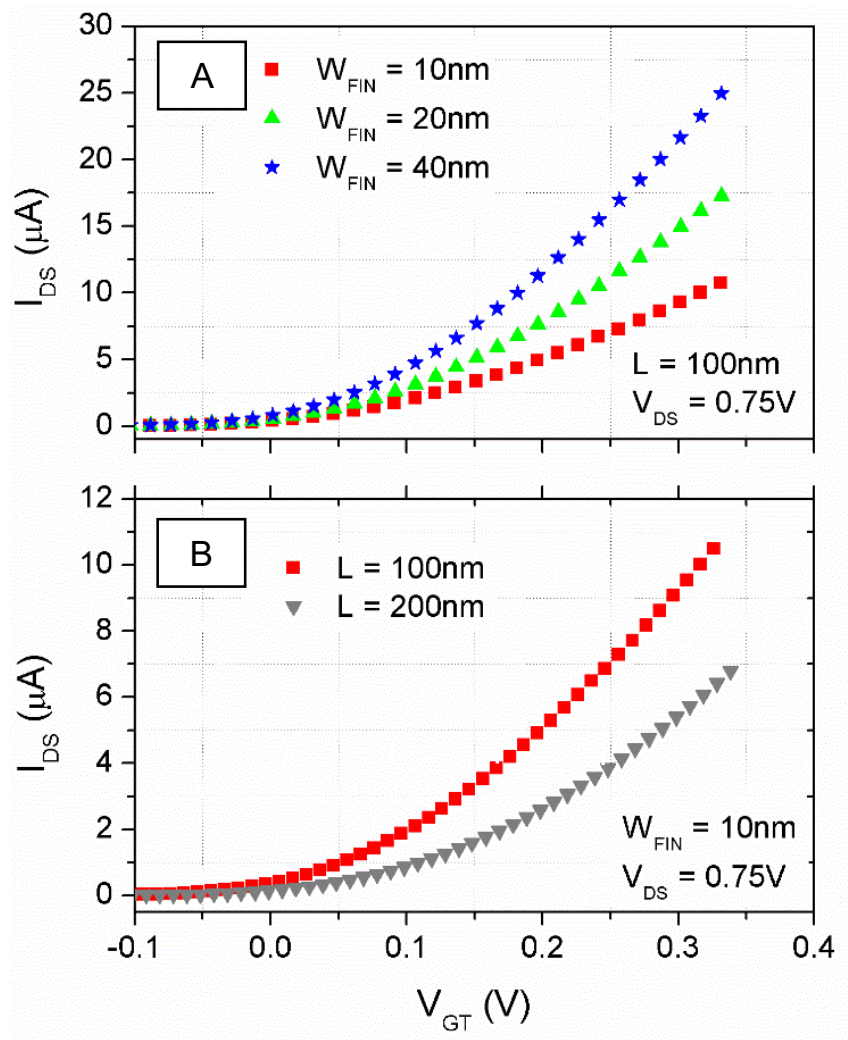

Fig.2 $I_{D S}$ as function of $V_{G T}$ curves, measured with $V_{D S}=0.75 V$, for stacked nanowires with $(\mathrm{A})$ variable $\mathrm{W}_{\mathrm{FIN}}(\mathrm{L}=100 \mathrm{~nm})$ and $(\mathrm{B})$ variable $\mathrm{L}$ $\left(\mathrm{W}_{\mathrm{FIN}}=10 \mathrm{~nm}\right)$.

The IFM method has been applied on these data, considering a voltage amplitude $\left(\mathrm{V}_{\mathrm{A}}\right)$ of $50 \mathrm{mV}$. The figures of merit considered were THD and third order harmonic distortion (HD3), which shows if even or odds harmonics are preponderant in this transistor architecture. Fig. 3 presents the resulting THD and HD3 as a function of $\mathrm{V}_{\mathrm{GT}}$ for all measured devices. Observing Fig. 3, one can see that the second order harmonic (HD2) is the major influencer of signal distortion for stacked nanowires: while THD varies between $-20 \mathrm{~dB}$ and $-27 \mathrm{~dB}$, HD3 represents only a parcel between $-50 \mathrm{~dB}$ and $-61 \mathrm{~dB}$.
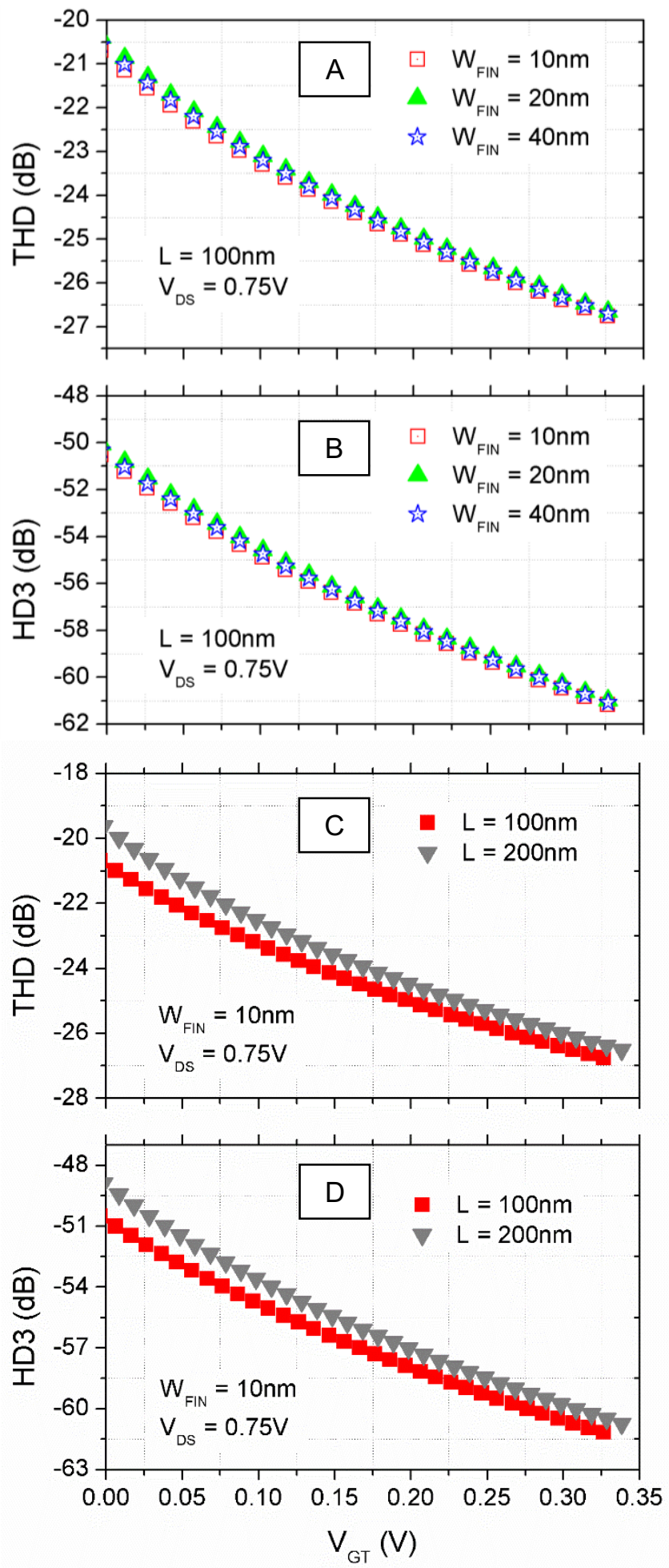

Fig.3 THD and HD3 as function of $\mathrm{V}_{\mathrm{GT}}$ curves, extracted with $\mathrm{V}_{\mathrm{DS}}=$ $0.75 \mathrm{~V}$ and $\mathrm{V}_{\mathrm{A}}=50 \mathrm{mV}$, for stacked nanowires with $(\mathrm{A})$ - (B) variable $\mathrm{W}_{\text {FIN }}$ $(\mathrm{L}=100 \mathrm{~nm})$, and $(\mathrm{C})$ - (D) variable $\mathrm{L}\left(\mathrm{W}_{\mathrm{FIN}}=10 \mathrm{~nm}\right)$, respectively. 
Although it is possible to verify a slightly difference of non-linearity for $\mathrm{L}=100 \mathrm{~nm}$ and $\mathrm{L}=200 \mathrm{~nm}, \mathrm{~W}_{\mathrm{FIN}}$ values did not show a major discretization with $\mathrm{V}_{\mathrm{GT}}$ variation.

Despite this fact, it is important to state that all results are in agreement with theoretical equation of HD2 [7], which demonstrates inversely proportional relation between harmonic distortion with overdrive gate voltage.

$$
\mathrm{HD} 2=\frac{\mathrm{V}_{\mathrm{A}}}{\mathrm{V}_{\mathrm{GT}}\left[2+3\left(\theta+\mathrm{R}_{\mathrm{S}} \mathrm{K}_{\text {low }}\right) \mathrm{V}_{\mathrm{GT}}+\left(\theta+\mathrm{R}_{\mathrm{S}} \mathrm{K}_{\text {low }}\right)^{2} \mathrm{~V}_{\mathrm{GT}}{ }^{2}\right]}
$$

In eqn. (2), $\theta$ is the mobility degradation factor, $R_{S}$ is the series resistance and $\mathrm{K}_{\text {low }}$ is the transistor gain described by eqn. (3), where $\mu_{0}$ is the low field mobility and $C_{O x}$ is the gate oxide capacitance per unit of area.

$\mathrm{K}_{\text {low }}=\frac{\mu_{0}}{1+\theta \mathrm{V}_{\mathrm{GT}}} \mathrm{C}_{\mathrm{OX}}\left(\frac{\mathrm{W}_{\text {(STACKED })}}{\mathrm{L}}\right)$

According to eqn. (2), for same $\mathrm{V}_{\mathrm{GT}}$ and $\mathrm{V}_{\mathrm{A}}$, the dominating aspects determining $\mathrm{HD} 2$ are $\theta, \mathrm{R}_{\mathrm{S}}$ and $\mathrm{K}_{\text {low. }}$. For longer $\mathrm{L}$ and similar $\mathrm{W}_{\mathrm{FIN}}$ there is a reduction in $\mathrm{K}_{\text {low }}$, which is the responsible for the slight increase of HD2 seen for $\mathrm{L}=200 \mathrm{~nm}$ with regards to $\mathrm{L}=100 \mathrm{~nm}$.

In order to get some insight on HD2 dependence on $\theta, \mathrm{R}_{\mathrm{S}}$ and $\mathrm{K}_{\text {low }}$, the experimental $\mathrm{I}_{\mathrm{DS}}$ versus $\mathrm{V}_{\mathrm{GS}}$ curves measured at $\mathrm{V}_{\mathrm{DS}}=25 \mathrm{mV}$ were taken. The extraction of $\mu_{0}$ and $\theta$ has been made using the method proposed in [8] whereas the $R_{S}$ extraction has been made with the method proposed in [9]. Table I presents the obtained results for $\mu_{0}, \theta$ and $\mathrm{R}_{\mathrm{S}}$ for the devices with variable $\mathrm{W}_{\mathrm{FIN}}$ and fixed $\mathrm{L}$ :

Table I. Extracted results of $\mu_{0}, \theta$ and $\mathrm{R}_{\mathrm{S}}$ for the measured stacked nanowires with variable $\mathrm{W}_{\text {FIN }}$ and fixed $\mathrm{L}$, biased at $\mathrm{V}_{\mathrm{DS}}=25 \mathrm{mV}$.

\begin{tabular}{cccc}
\hline $\mathrm{W}_{\text {FIN }}(\mathrm{nm})$ & $\begin{array}{c}\mu_{0} \\
\left(\mathrm{~cm}^{2} / \mathrm{Vs}\right)\end{array}$ & $\begin{array}{c}\theta \\
\left(\mathrm{V}^{-1}\right)\end{array}$ & $\begin{array}{c}\mathrm{R}_{\mathrm{S}} \mathrm{W} \\
\left(\Omega^{*} \mu \mathrm{m}\right)\end{array}$ \\
\hline 10 & 105.8 & 0.56 & 1391 \\
\hline 20 & 101.4 & 0.35 & 1229 \\
\hline 40 & 116.6 & 0.34 & 1413 \\
\hline
\end{tabular}

The results presented in Table I indicate that the terms $\theta$ and $\mathrm{R}_{\mathrm{S}} . \mathrm{K}_{\text {low }}$, which compose the denominator of eqn. (2), are of the same magnitude in the studied devices. This way, there is a balanced contribution of them in HD2 with no clear dominance of any.

As a result of low data discretization, another strategy was used: observation of distortions as function of $\mathrm{g}_{\mathrm{m}} / \mathrm{I}_{\mathrm{DS}}$. This propitiates an analysis with all devices biased on the same operational region, excluding $\mathrm{V}_{\mathrm{GT}}$ dependency.

These new comparisons are found in Figs. 4 and 5, that present the THD and HD3 of measured devices as a function of $g_{m} / I_{D S}$ for fixed $L$ and $W_{F I N}$, respectively. Once again values are close together with each other, but it is possible to establish somewhat resemblance with THD and the different $\mathrm{W}_{\text {FIN }}$ of transistors: $\mathrm{W}_{\mathrm{FIN}}=40 \mathrm{~nm}$ has the largest non-linearity amongst other devices. All of them present a lower distortion with reduction of $\mathrm{g}_{\mathrm{m}} / \mathrm{I}_{\mathrm{DS}}$, in accordance with previously results found in Fig. 3 and eqn. (2), since the $\mathrm{V}_{\mathrm{GT}}$ increase leads to smaller $\mathrm{g}_{\mathrm{m}} / \mathrm{I}_{\mathrm{DS}}$ values.
For the devices with variable $L$ biased at the same $g_{m} / I_{D S}$, one can see that THD and HD3 are weakly dependent on L. in the studied bias range.
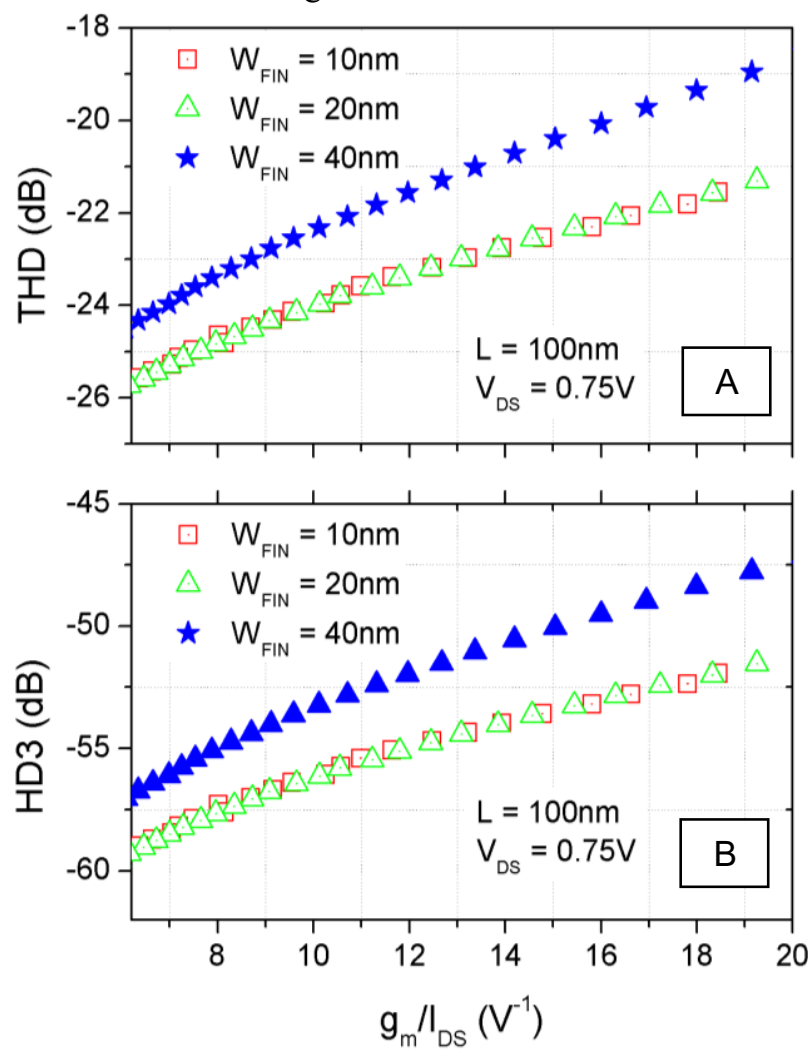

Fig.4 Extracted (A) THD and (B) HD3 as function of $g_{m} / I_{D s}$ curves for stacked nanowires with variable $\mathrm{W}_{\mathrm{FIN}}(\mathrm{L}=100 \mathrm{~nm})$ at $\mathrm{V}_{\mathrm{DS}}=0.75 \mathrm{~V}$ and
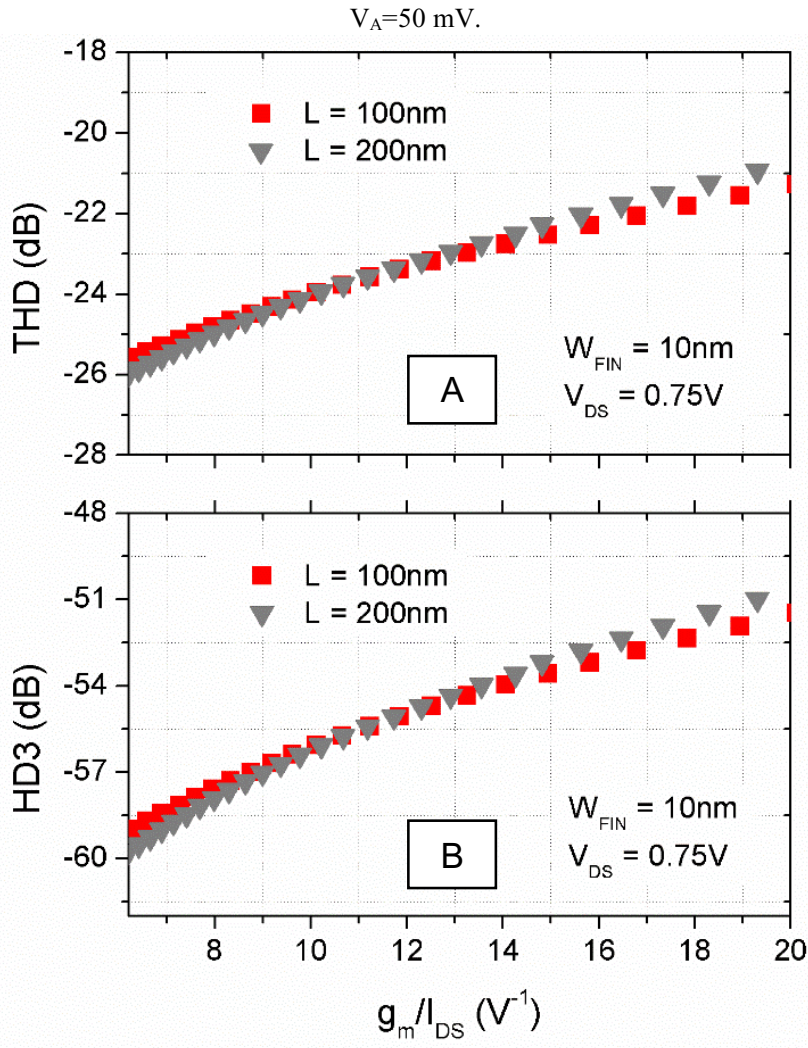

Fig.5 Extracted (A) THD and (B) HD3 as function of $\mathrm{g}_{\mathrm{m}} / \mathrm{I}_{\mathrm{DS}}$ curves for stacked nanowires with variable $\mathrm{L}\left(\mathrm{W}_{\mathrm{FIN}}=10 \mathrm{~nm}\right)$ at $\mathrm{V}_{\mathrm{DS}}=0.75 \mathrm{~V}$ and $\mathrm{V}_{\mathrm{A}}=50 \mathrm{mV}$. 
From Fig. 5 it is possible to see that for the devices with variable $\mathrm{L}$ biased at the same $\mathrm{g}_{\mathrm{m}} / \mathrm{I}_{\mathrm{DS}}$, THD and HD3 are weakly dependent on $\mathrm{L}$ in the studied bias range.

An even better perspective can be achieved by using intrinsic gain voltage $\left(\mathrm{A}_{\mathrm{V}}\right)$ extracted in [2]. Tables II and III associate different widths and lengths with their respective values for $\mathrm{V}_{\mathrm{GT}}, \mathrm{g}_{\mathrm{m}} / \mathrm{I}_{\mathrm{DS}}$ and $\mathrm{A}_{\mathrm{V}}$.

Table II. Correlation between $\mathrm{V}_{\mathrm{GT}}, \mathrm{g}_{\mathrm{m}} / \mathrm{I}_{\mathrm{DS}}$ and $\mathrm{A}_{\mathrm{V}}$ for the stacked nanowires with variable $\mathrm{W}_{\mathrm{FIN}}$ and $\mathrm{L}=100 \mathrm{~nm}$.

\begin{tabular}{cccc}
\hline \multicolumn{4}{c}{ nanowires with variable $\mathrm{W}_{\mathrm{FIN}}$ and $/=100 \mathrm{~nm}}$. \\
\hline $\mathrm{W}_{\mathrm{FIN}}(\mathrm{nm})$ & $\mathrm{V}_{\mathrm{GT}}(\mathrm{mV})$ & $\mathrm{g}_{\mathrm{m}} / \mathrm{I}_{\mathrm{DS}}\left(\mathrm{V}^{-1}\right)$ & $\mathrm{A}_{\mathrm{V}}(\mathrm{dB})$ \\
\multirow{3}{*}{10} & 100 & 12.04 & 41.34 \\
& 200 & 7.53 & 37.83 \\
& 300 & 5.00 & 32.33 \\
\hline \multirow{2}{*}{20} & 100 & 13.02 & 41.09 \\
& 200 & 7.91 & 37.94 \\
& 300 & 5.17 & 32.67 \\
\hline \multirow{3}{*}{40} & 100 & 12.70 & 38.08 \\
& 200 & 8.06 & 35.83 \\
& 300 & 5.12 & 32.09 \\
\hline
\end{tabular}

Table III. Correlation between $\mathrm{V}_{\mathrm{GT}}, \mathrm{g}_{\mathrm{m}} / \mathrm{I}_{\mathrm{DS}}$ and $\mathrm{A}_{\mathrm{V}}$ for the stacked nanowires with $\mathrm{W}_{\mathrm{FIN}}=10 \mathrm{~nm}$ and variable $\mathrm{L}$.

\begin{tabular}{cccc}
\hline $\mathrm{L}(\mathrm{nm})$ & $\mathrm{V}_{\mathrm{GT}}(\mathrm{mV})$ & $\mathrm{g}_{\mathrm{m}} / \mathrm{I}_{\mathrm{DS}}\left(\mathrm{V}^{-1}\right)$ & $\mathrm{A}_{\mathrm{V}}(\mathrm{dB})$ \\
\hline \multirow{3}{*}{100} & 100 & 12.04 & 41.34 \\
& 200 & 7.53 & 37.83 \\
& 300 & 5.00 & 32.33 \\
\hline \multirow{2}{*}{200} & 100 & 13.68 & 53.55 \\
& 200 & 8.79 & 42.60 \\
& 300 & 6.21 & 34.75 \\
\hline
\end{tabular}

All devices show similar comportment, decreasing the gain with higher gate voltage overdrive. Such tendency is expected considering that in this voltage range the stacked nanowires are moving towards moderate to strong inversion region, exhibiting a decrease in $A_{V}$ as previously reported in [10]. As the devices have different $A_{V}$, the amplitude of the output signal with similar input signal amplitude will be different, which could be pointed as the cause of the larger distortion amongst them.

To decorrelate the different $A_{V}$ and the distortion data, Fig. 6 presents the THD/Av and HD3/Av as function of $\mathrm{g}_{\mathrm{m}} / \mathrm{I}_{\mathrm{DS}}$ for devices with constant $\mathrm{L}$ and $\mathrm{W}_{\text {FIN }}$.

The direction of the curves has changed as a result of the lower $A_{V}$ values as $g_{m} / I_{D s}$ reduces. Besides, the main contribution for new plots is the completely separated points, facilitating the analysis.

Finally, one last comparation can be made to get insight on the reasons for the different THD. It is known that the HD2 can be obtained by the derivative of the transconductance, as indicated in eqn. (4) [11]:

$\mathrm{HD} 2=\frac{\mathrm{V}_{\mathrm{A}}}{4} \frac{\frac{\delta \mathrm{g}_{\mathrm{m}}}{\delta \mathrm{V}_{\mathrm{GT}}}}{\mathrm{g}_{\mathrm{m}}}$

Fig. 7 presents the calculated $\mathrm{dg}_{\mathrm{m}} / \mathrm{dV}_{\mathrm{GT}}$ as a function of $\mathrm{g}_{\mathrm{m}} / \mathrm{I}_{\mathrm{DS}}$ for the experimental devices with variable $\mathrm{W}_{\mathrm{FIN}}$, biased with $\mathrm{V}_{\mathrm{DS}}=0.75 \mathrm{~V}$ and $\mathrm{V}_{\mathrm{A}}=50 \mathrm{mV}$.
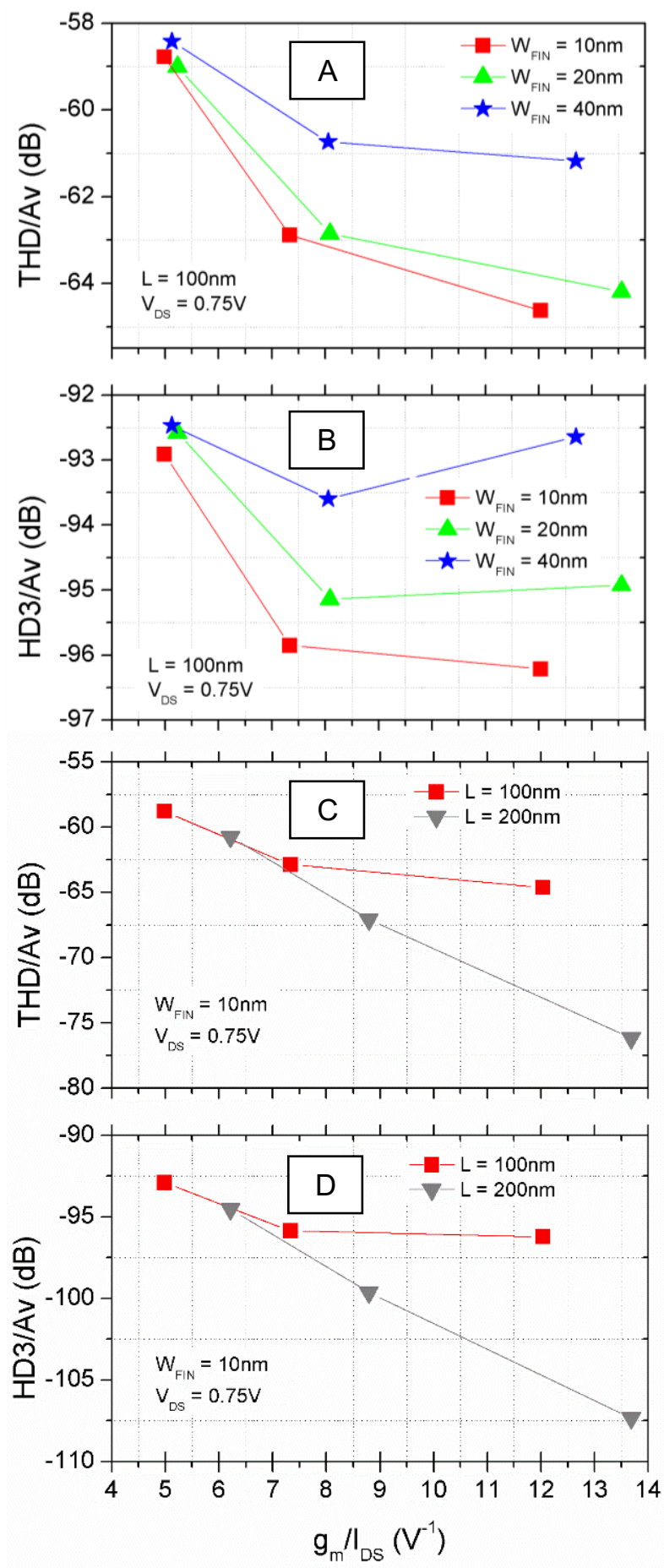

Fig.6 THD/A $/ \mathrm{A}_{V}$ and HD3/ $\mathrm{A}_{\mathrm{V}}$ as function of $\mathrm{g}_{\mathrm{m}} / \mathrm{I}_{\mathrm{DS}}$ curves extracted with $\mathrm{V}_{\mathrm{DS}}=0.75 \mathrm{~V}$ and $\mathrm{V}_{\mathrm{A}}=50 \mathrm{mV}$, for stacked nanowires with (A) - (B) variable

$\mathrm{W}_{\mathrm{FIN}}(\mathrm{L}=100 \mathrm{~nm})$, and $(\mathrm{C})$ - (D) variable $\mathrm{L}\left(\mathrm{W}_{\mathrm{FIN}}=10 \mathrm{~nm}\right)$, respectively.

By plotting $d g_{m} / d V_{G T}$ as function of $g_{m} / I_{D S}$ it is possible to see that $\mathrm{W}_{\mathrm{FIN}}=40 \mathrm{~nm}$, which has larger fin width among the devices, has the greatest variation and is also the one with higher THD, aligned with Fig. 4, eqn. (4).

The results presented in this work can be compared with those from [12], which studies single level nanowires with variable $W_{\text {FIN }}$ and similar gate stack, although the channel length and $V_{D S}$ are different. The fin width dependence is similar, showing that wider devices presented worst linearity than narrower ones because of the larger $\mathrm{dg}_{\mathrm{m}} / \mathrm{dV}_{\mathrm{GT}}$. 


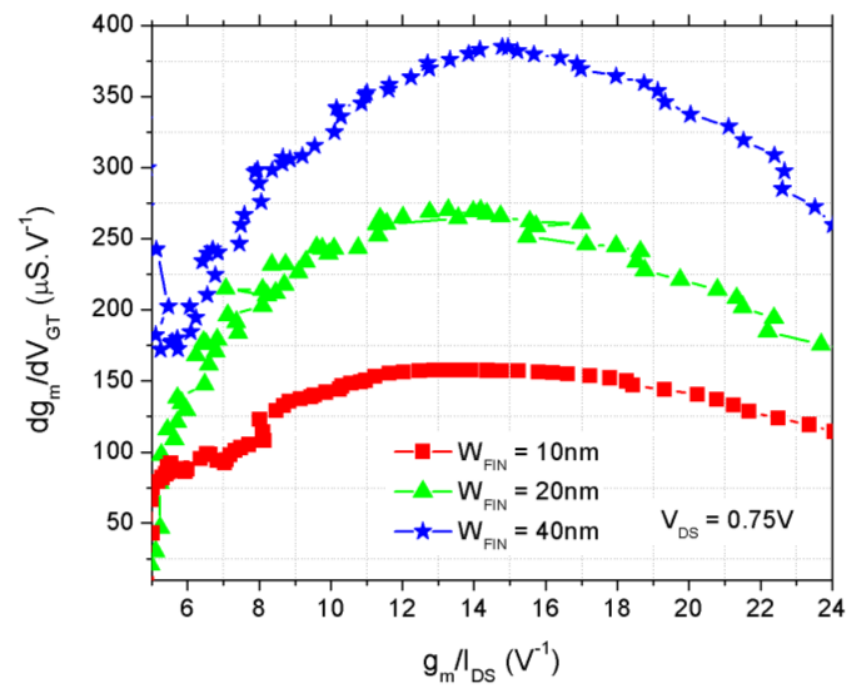

Fig.7 Calculated $\mathrm{dg}_{\mathrm{m}} / \mathrm{dV}_{\mathrm{GT}}$ as function of $\mathrm{g}_{\mathrm{m}} / \mathrm{I}_{\mathrm{DS}}$ curves for the stacked nanowire transistors with $\mathrm{L}=100 \mathrm{~nm}$ and variable $\mathrm{W}_{\mathrm{FIN}}$, biased with $\mathrm{V}_{\mathrm{DS}}=0.75 \mathrm{~V}$ and $\mathrm{V}_{\mathrm{A}}=50 \mathrm{mV}$.

Regarding THD and HD3, one can notice that for $\mathrm{V}_{\mathrm{GT}}=300 \mathrm{mV}$, both stacked and single level nanowires present THD around $-26.5 \mathrm{~dB}$. Observing THD versus $\mathrm{g}_{\mathrm{m}} / \mathrm{I}_{\mathrm{DS}}$ equal to $12 \mathrm{~V}^{-1}$, the non-linearity of vertically stacked nanowire varies between $-23.5 \mathrm{~dB}$ and $-22 \mathrm{~dB}$ (Fig. 4). On the other hand, for the single level nanowire transistor, this value is around $-25 \mathrm{~dB}$ for $\mathrm{W}_{\mathrm{FIN}}=9.5 \mathrm{~nm}$.

\section{Conclusions}

This paper presented the harmonic distortion of 2-level stacked nanowires with variable fin width and channel length. For all studied transistors, the harmonic distortion is dominated by the second order harmonic distortion, with $30 \mathrm{~dB}$ larger than the third order harmonic distortion. After decorrelating the harmonic distortion from the device intrinsic voltage gain, it has been demonstrated that devices with wide fin width presented larger harmonic distortion than narrow ones, in all operational regions. It is caused by the larger variation of the transconductance with the gate voltage overdrive. Also, longer devices present greater harmonic distortion than short ones.

\section{ACKNOWLEDGEMENTS}

The authors would like to thank Prof. Antonio Cerdeira, from CINVESTAV, Mexico, for his help regarding the IFM Method, which was essential in this work. This work was supported by Coordenação de Aperfeiçoamento de Pessoal de Nível Superior - Brazil (CAPES) - Finance Code 001, $\mathrm{CNPq}$ and Sao Paulo Research Foundation (FAPESP grant \# 2019/15500-5). Also, this paper has been supported by the French Public Authorities from NANO 2017 program. This work is also partially funded by SUPERAID7 (grant $\mathrm{N}^{\circ}$ 688101) project.

\section{REFERENCES}

[1] E. Bernard, et al., "Novel integration process and performances analysis of low standby power (LSTP) 3D multi-channel MOSFET (MCFET) on SOI with metal/high-k gate stack," VLSI, pp. 16-17, 2008.

[2] G. Mariniello, et al., "Evaluation of Analog Characteristics of n-Type Vertically Stacked Nanowires," accepted for 2020 EUROSOI-ULIS.

[3] N. Mehta, et al., "A 1-mW Class-AB Amplifier With -101 dB THD+N for High-Fidelity 16 Omega Headphones in 65-nm CMOS," in IEEE Journal of Solid-State Circuits, vol. 54, no. 4, pp. 948-958, April 2019, doi: 10.1109/JSSC.2018.2886320.

[4] Y.Song, et al., "Vertically stacked individually tunable nanowire field effect transistors for low power operation with ultrahigh radio frequency linearity," 2012. Applied Physics Letters. 101. 10.1063/1.4747448

[5] S. Barraud, et al., "Tunability of Parasitic Channel in Gate-All-Around Stacked Nanosheets," 2018 IEEE International Electron Devices Meeting (IEDM), San Francisco, CA, 2018, pp. 21.3.1-21.3.4, doi: 10.1109/IEDM.2018.8614507.

[6] A. Cerdeira, et al., "Integral function method for determination of nonlinear harmonic distortion," Solid-State Electron., vol. 48, no. 12, pp. 2225-2234, 2004.

[7] R. T. Doria et al., "Harmonic Distortion of Unstrained and Strained FinFETs Operating in Saturation," in IEEE Transactions on Electron Devices, vol. 57, no. 12, pp. 3303-3311, Dec. 2010, doi: 10.1109/TED.2010.2079936.

[8] A. Dixit et al., "Analysis of the parasitic S/D resistance in multiplegate FETs," in IEEE Trans. Electron Devices, vol. 52, no. 6, pp. 1132 1140, Jun. 2005. doi: 10.1109/TED.2005.848098

[9] G. Ghibaudo, "New method for the extraction of MOSFET parameters," in Electronics Letters, vol. 24, no. 9, pp. 543-545, Apr. 1988. doi: $10.1049 / \mathrm{el}: 19880369$

[10] F. Silveira, et al., "A $\mathrm{g}_{\mathrm{m}} / / \mathrm{I}_{\mathrm{D}}$ based methodology for the design of CMOS analog circuits and its application to the synthesis of a siliconon-insulator micropower OTA," in IEEE Journal of Solid-State Circuits, vol. 31, no. 9, pp. 1314-1319, Sept. 1996, doi: $10.1109 / 4.535416$.

[11] G. Groenewold and W. J. Lubbers, "Systematic distortion analysis for MOSFET integrators with use of a new MOSFET model," in IEEE Trans. Circuits Syst. II, Analog Digit. Signal Process., vol. 41, no. 9, pp. 569-580, Sept. 1994. doi: 10.1109/82.326583.

[12] B. C. Paz, et al., "Non-linearity analysis of triple gate SOI nanowires MOSFETS," 2016 31st Symposium on Microelectronics Technology and Devices (SBMicro), Belo Horizonte, 2016, pp. 1-4, doi: 10.1109/SBMicro.2016.7731355. 